\title{
Switchable Synthesis of Aryl Sulfones and Sulfoxides through Solvent-Promoted Oxidation of Sulfides with $\mathrm{O}_{2} / \mathrm{Air}$
}

\author{
Zhen Cheng, Pengchao Sun, Ailing Tang, Weiwei Jin* and Chenjiang Liu* \\ The Key Laboratory of Oil and Gas Fine Chemicals, Ministry of Education \& Xinjiang Uygur \\ Autonomous Region, Urumqi Key Laboratory of Green Catalysis and Synthesis Technology, School of \\ Chemistry and Chemical Engineering, Xinjiang University, Urumqi 830046, P. R. China. \\ E-mail:wwjin0722@163.com;pxylcj@126.com
}

\section{Table of Contents}

$\begin{array}{ll}\text { 1. Materials and methods } & \text { S2 }\end{array}$

$\begin{array}{ll}\text { 2. Experimental procedures } & \text { S3 }\end{array}$

2.1 General procedure for synthesis of substrates $\quad$ S3

2.2 General procedure for synthesis of products $\quad$ S3

2.3 Synthetic application of sulfones and sulfoxides $\quad$ S4

3. Mechanistic studies

3.1 Radical trapping experiments S5

3.2 Isotope labeling experiments $\quad$ S6

$\begin{array}{ll}3.3 \text { Intermediate probe experiment } & \text { S8 }\end{array}$

3.4 Eelectron paramagnetic resonance (EPR) experiment $\quad$ S9

$\begin{array}{ll}3.5 \text { In-situ IR experiment } & \text { S9 }\end{array}$

$\begin{array}{ll}\text { 4. Analytical data } & \text { S11 }\end{array}$

$\begin{array}{ll}\text { 5. References } & \text { S23 }\end{array}$

$\begin{array}{ll}\text { 6. Copies of NMR spectra } & \text { S25 }\end{array}$ 


\section{Materials and methods}

General. ${ }^{1} \mathrm{H}$ and ${ }^{13} \mathrm{C}$ NMR spectra were recorded on a Varian Inova-400 (400 $\mathrm{MHz}$ and $100 \mathrm{MHz}$, respectively) spectrometer. ${ }^{1} \mathrm{H}$ and ${ }^{13} \mathrm{C}$ NMR chemical shifts were determined relative to internal standard TMS at $\delta 0.0, \mathrm{CDCl}_{3}\left(\delta\left({ }^{1} \mathrm{H}\right), 7.26 \mathrm{ppm}\right.$; $\left.\delta\left({ }^{13} \mathrm{C}\right), 77.16 \mathrm{ppm}\right)$ or DMSO- $d_{6}\left(\delta\left({ }^{1} \mathrm{H}\right), 2.50 \mathrm{ppm} ; \delta\left({ }^{13} \mathrm{C}\right), 39.52 \mathrm{ppm}\right)$. Chemical shifts $(\delta)$ are reported in ppm, and coupling constants $(J)$ are in Hertz $(\mathrm{Hz})$. The following abbreviations are used to explain the multiplicities: $\mathrm{s}=$ singlet, $\mathrm{d}=$ doublet, $\mathrm{t}=$ triplet, $\mathrm{q}=$ quartet, $\mathrm{m}=$ multiplet, $\mathrm{br}=$ broad. The HRMS analysis was obtained on a Agilent 6540 UHD Q-TOF mass spectrometer. The melting point was recorded on BÜCHI (M-560) and uncorrected. IR spectra were recorded on a Mettler Toledo React IR I5. EPR spectra were recorded on a JEOL JES-FA200 spectrometer. Analytical thin layer chromatography (TLC) was performed on $0.25 \mathrm{~mm}$ silica gel 60 F254 plates and viewed by UV light ( $254 \mathrm{~nm})$. Column chromatographic purification was performed using 200-300 mesh silica gel.

Materials. All the chemical reagents were purchased from commercial sources and used as received unless otherwise indicated. Starting materials $\mathbf{1 b - c}$ are known compounds and synthesized according to the reported method. ${ }^{1}$ Their spectroscopic features are in good agreement with those reported in the literatures. 


\section{Experimental procedures}

\subsection{General procedure for synthesis of substrates}

\subsubsection{A typical procedure for the synthesis of $1 \mathrm{~b}$}

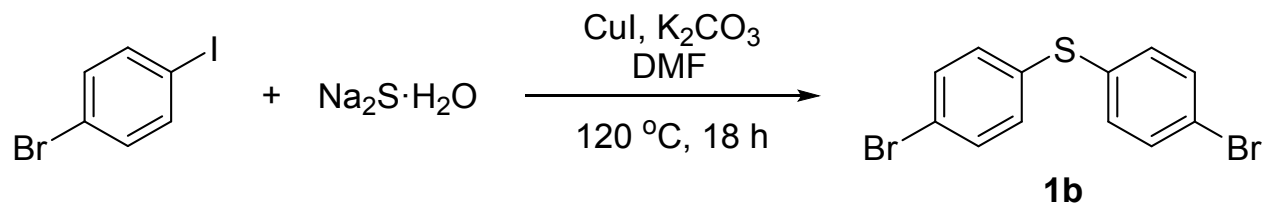

A flame-dried test tube containing a magnetic stirring bar was charged with $\mathrm{CuI}$ (0.2 mmol), $\mathrm{K}_{2} \mathrm{CO}_{3}(2 \mathrm{mmol}), \mathrm{Na}_{2} \mathrm{~S}(1.2 \mathrm{mmol})$, the 1-bromo-4-iodobenzene (2.0 $\mathrm{mmol}$ ), and DMF (4 mL) under argon. The mixture was heated at $120^{\circ} \mathrm{C}$ for $18 \mathrm{~h}$ and allowed to cool to room temperature. The resulting mixture was extracted with ethyl acetate $(3 \times 25 \mathrm{~mL})$. The combined organic layers were dried with $\mathrm{Na}_{2} \mathrm{SO}_{4}$ and then concentrated under vacuum. The residue was purified by column chromatography on silica gel with an eluent consisting of petroleum ether and ethyl acetate.

\subsubsection{A typical procedure for the synthesis of 1c}

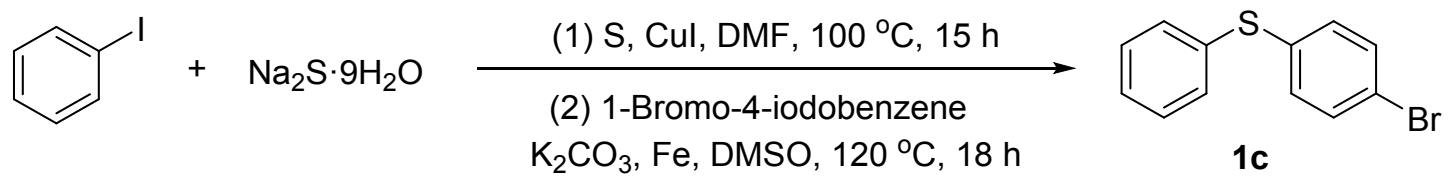

A flame-dried test tube containing a magnetic stirring bar was charged with $\mathrm{CuI}$ (0.1 mmol), $\mathrm{Na}_{2} \mathrm{~S}(1.0 \mathrm{mmol}), \mathrm{S}(1.0 \mathrm{mmol})$, iodobenzene $(1.0 \mathrm{mmol})$, and DMF (2 $\mathrm{mL}$ ) under argon. The mixture was heated at $100{ }^{\circ} \mathrm{C}$ for $15 \mathrm{~h}$. The reaction mixture was allowed to cool to room temperature, and the 1-bromo-4-iodobenzene $(1.6 \mathrm{mmol})$, Fe $(0.6 \mathrm{mmol}), \mathrm{K}_{2} \mathrm{CO}_{3}(2 \mathrm{mmol})$, and DMSO $(4 \mathrm{~mL})$ were added. The mixture was further heated under argon at $120{ }^{\circ} \mathrm{C}$ for $18 \mathrm{~h}$ and allowed to cool to room temperature. The resulting mixture was extracted with ethyl acetate $(3 \times 25 \mathrm{~mL})$. The combined organic layers were dried with $\mathrm{Na}_{2} \mathrm{SO}_{4}$ and then concentrated under vacuum. The residue was purified by column chromatography on silica gel with an eluent consisting of petroleum ether and ethyl acetate.

\subsection{General procedure for synthesis of products (taking $2 \mathrm{a}$ and $3 \mathrm{a}$ as examples)}




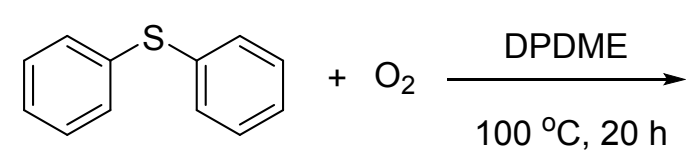

$1 a$<smiles>O=S(=O)(c1ccccc1)c1ccccc1</smiles>

2a

A mixture of diphenyl sulfide 1a $(186 \mathrm{mg}, 1.0 \mathrm{mmol})$ and DPDME (1.0 mL) were added to a $10 \mathrm{~mL}$ Schlenk tube. The reaction tube was flushed with $\mathrm{O}_{2}$ for 1.0 minute and then equipped with a $\mathrm{O}_{2}$ balloon. The reaction was stirred at $100{ }^{\circ} \mathrm{C}$ for 20 h. After cooling to ambient temperature, the resulting mixture was extracted with EtOAc $(3 \times 10 \mathrm{~mL})$. The combined organic phase was dried over anhydrous $\mathrm{MgSO}_{4}$, filtered, and all the volatiles were evaporated under reduced pressure. The resultant residue was purified by silica gel column chromatography (eluent: petroleum ether $\left.\left(60-90{ }^{\circ} \mathrm{C}\right) / \mathrm{EtOAc}=25: 1, \mathrm{v} / \mathrm{v}\right)$ to afford the desired product $\mathbf{2 a}$ as a white solid $(212$ mg, 97\% yield).

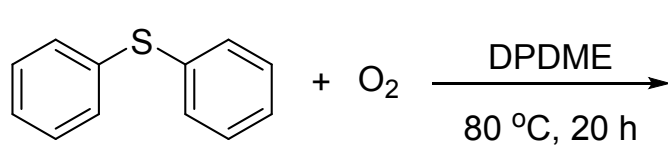

1a<smiles>O=S(c1ccccc1)c1ccccc1</smiles>

3a

A mixture of diphenyl sulfide 1a $(186 \mathrm{mg}, 1.0 \mathrm{mmol})$ and DPDME $(1.0 \mathrm{~mL})$ were added to a $10 \mathrm{~mL}$ reaction tube. The reaction was stirred at $80{ }^{\circ} \mathrm{C}$ for $20 \mathrm{~h}$. After cooling to ambient temperature, the resulting mixture was extracted with EtOAc $(3 \times 10 \mathrm{~mL})$. The combined organic phase was dried over anhydrous $\mathrm{MgSO}_{4}$, filtered, and all the volatiles were evaporated under reduced pressure. The resultant residue was purified by silica gel column chromatography (eluent: petroleum ether (60-90 $\left.\left.{ }^{\circ} \mathrm{C}\right) / \mathrm{EtOAc}=10: 1, \mathrm{v} / \mathrm{v}\right)$ to afford the desired product 3a as a white solid (142 $\mathrm{mg}$, $70 \%$ yield) (2a was isolated in $6 \%, 13 \mathrm{mg}$ ).

\subsection{Synthetic application of sulfones and sulfoxides}

In order to highlight the synthetic importance of sulfoxides and sulfones in organic chemistry, several useful transformations were realized according to the known methods reported in literatues and the desired products ester $4,{ }^{2 a}$ trans-stilbene $5^{2 b}$, and arylated ketone $6^{2 \mathrm{c}}$, sulfoximine $7^{2 \mathrm{~d}}$ were furnished in generally good yields. 


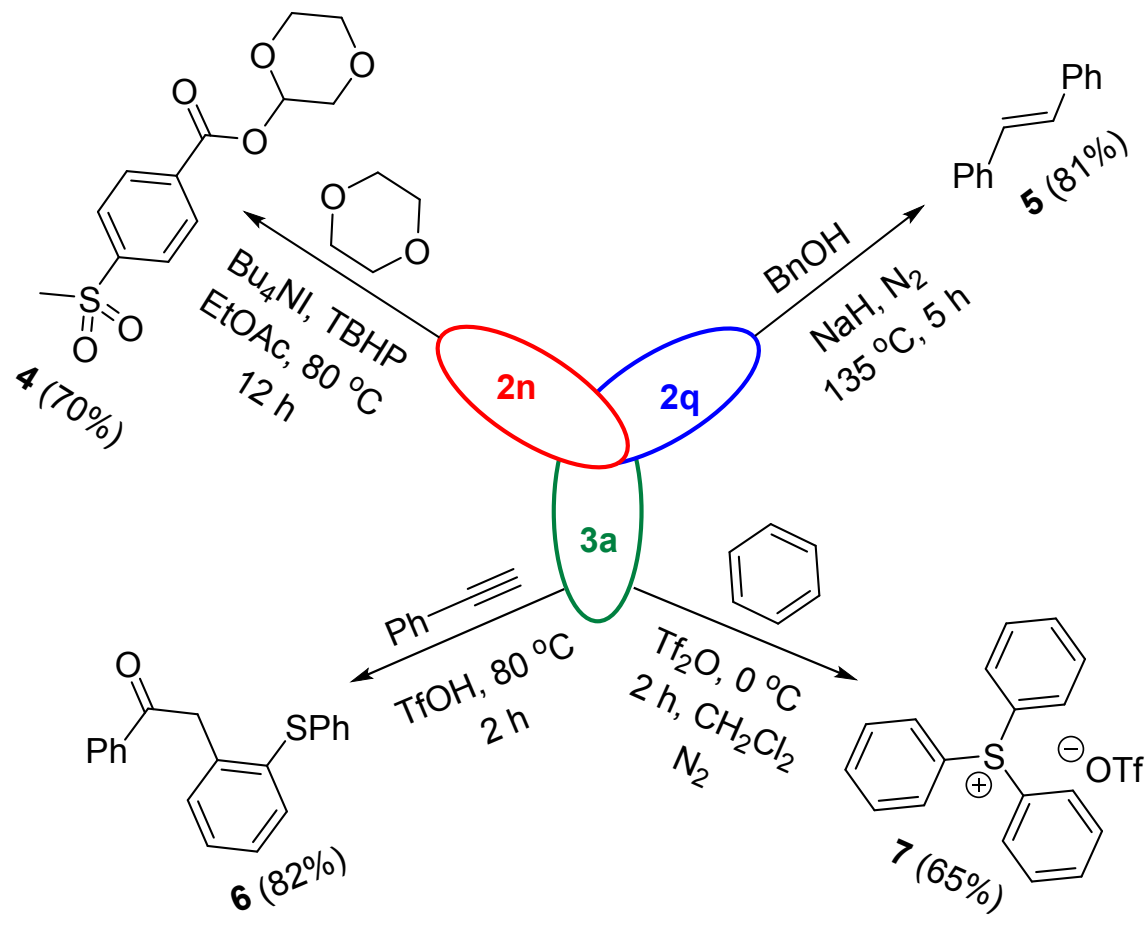

Scheme S1. Synthetic Application of Sulfones and Sulfoxides

\section{Mechanistic studies}

\subsection{Radical trapping experiments}<smiles>O=[N+]([O-])c1ccc(Sc2ccccc2)cc1</smiles>

$1 \mathrm{a}$

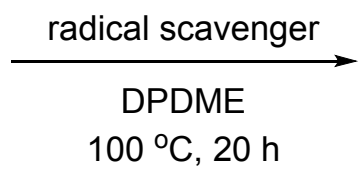

$100^{\circ} \mathrm{C}, 20 \mathrm{~h}$<smiles>O=S(c1ccccc1)c1ccccc1</smiles>

$2 a$
$3 a$

\begin{tabular}{|c|c|c|c|}
\hline entry & $\begin{array}{c}\text { radical scavenger } \\
\text { (equiv) }\end{array}$ & yield of 2a (\%) & yield of 3a (\%) \\
\hline 1 & TEMPO (2.0) & 0 & 0 \\
\hline 2 & BHT (2.0) & 0 & 0 \\
\hline 3 & 1,1 -diphenylethylene (2.0) & 0 & 0 \\
\hline
\end{tabular}

A mixture of diphenyl sulfide 1a (93 mg, $0.50 \mathrm{mmol}$ ), radical scavenger TEMPO, BHT or 1,1-diphenylethylene $(1.0 \mathrm{mmol})$ and DPDME $(0.5 \mathrm{~mL})$ were added to a 10 $\mathrm{mL}$ Schlenk tube. The reaction tube was flushed with $\mathrm{O}_{2}$ for 1.0 minute and then equipped with a $\mathrm{O}_{2}$ balloon. The reaction was stirred at $100{ }^{\circ} \mathrm{C}$ for $20 \mathrm{~h}$. After cooling to ambient temperature, the resulting mixture was detected by TLC and GC analysis.

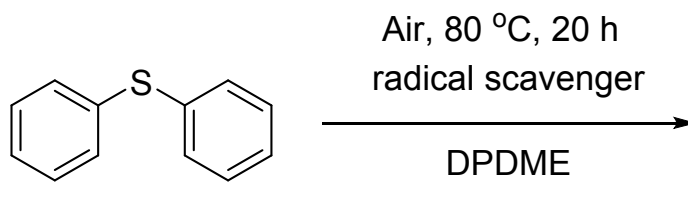

$1 \mathrm{a}$<smiles>O=S(=O)(c1ccccc1)c1ccccc1</smiles>

$2 a$<smiles>O=S(c1ccccc1)c1ccccc1</smiles>

3a 


\begin{tabular}{|c|c|c|c|}
\hline entry & $\begin{array}{c}\text { radical scavenger } \\
\text { (equiv) }\end{array}$ & yield of 2a (\%) & yield of 3a (\%) \\
\hline 1 & TEMPO (2.0) & 0 & 0 \\
\hline 2 & BHT (2.0) & 0 & 0 \\
\hline 3 & 1,1 -diphenylethylene (2.0) & 0 & 0 \\
\hline
\end{tabular}

A mixture of diphenyl sulfide $1 \mathrm{a}(93 \mathrm{mg}, 0.50 \mathrm{mmol})$, radical scavenger TEMPO, BHT or 1,1-diphenylethylene $(1.0 \mathrm{mmol})$ and DPDME $(0.5 \mathrm{~mL})$ were added to a 10 $\mathrm{mL}$ Reaction tube. The reaction was stirred at $80{ }^{\circ} \mathrm{C}$ for $20 \mathrm{~h}$. After cooling to ambient temperature, the resulting mixture was detected by TLC and GC analysis.

\subsection{Isotope labeling experiments}

\subsubsection{Isotope labeling experiment with ${ }^{18} \mathrm{O}_{2}$}

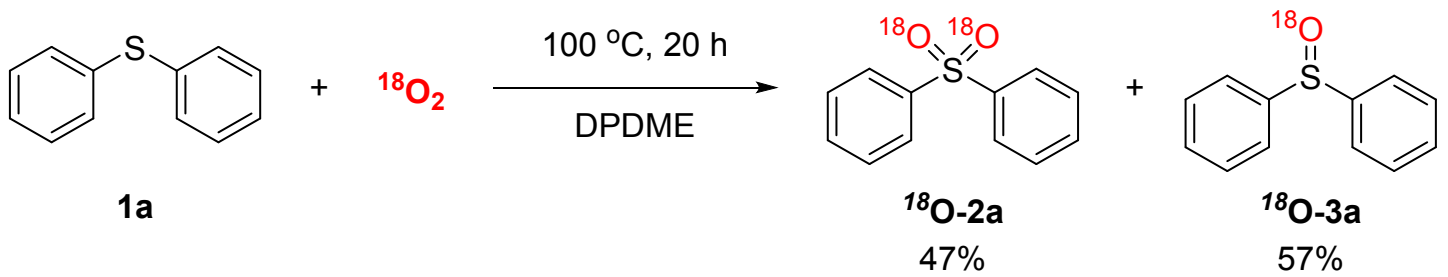

A mixture of diphenyl sulfide 1a $(93 \mathrm{mg}, 0.50 \mathrm{mmol})$ and DPDME $(0.5 \mathrm{~mL})$ were added to a $10 \mathrm{~mL}$ Schlenk tube. The reaction tube was then equipped with a ${ }^{18} \mathrm{O}_{2}$ balloon. The reaction was stirred at $100{ }^{\circ} \mathrm{C}$ for $20 \mathrm{~h}$. After cooling to ambient temperature, the resulting mixture was extracted with EtOAc $(3 \times 10 \mathrm{~mL})$. The combined organic phase was dried over anhydrous $\mathrm{MgSO}_{4}$, filtered, and all the volatiles were evaporated under reduced pressure. The resultant residue was purified by silica gel column chromatography (eluent: petroleum ether $\left(60-90{ }^{\circ} \mathrm{C}\right) / \mathrm{EtOAc}=$ $25: 1, \mathrm{v} / \mathrm{v})$ to afford the desired products ${ }^{18} \boldsymbol{O}-\mathbf{2 a}$ and ${ }^{18} \boldsymbol{O}-3 \mathbf{a}$ in $47 \%$ and $57 \%$ yields, respectively. Then the products were analyzed by HRMS.

HRMS of ${ }^{18} \boldsymbol{O}-\mathbf{2 a}$ : calcd for $\mathrm{C}_{12} \mathrm{H}_{9}{ }^{18} \mathrm{O}_{2} \mathrm{~S}$ [M-H]": 221.0408 ; found: 221.0413 .

HRMS of ${ }^{18} \boldsymbol{O}-3 a$ : calcd for $\mathrm{C}_{12} \mathrm{H}_{9}{ }^{18} \mathrm{OS}$ [M-H] $]^{-:}$203.0417; found: 203.0422 . 


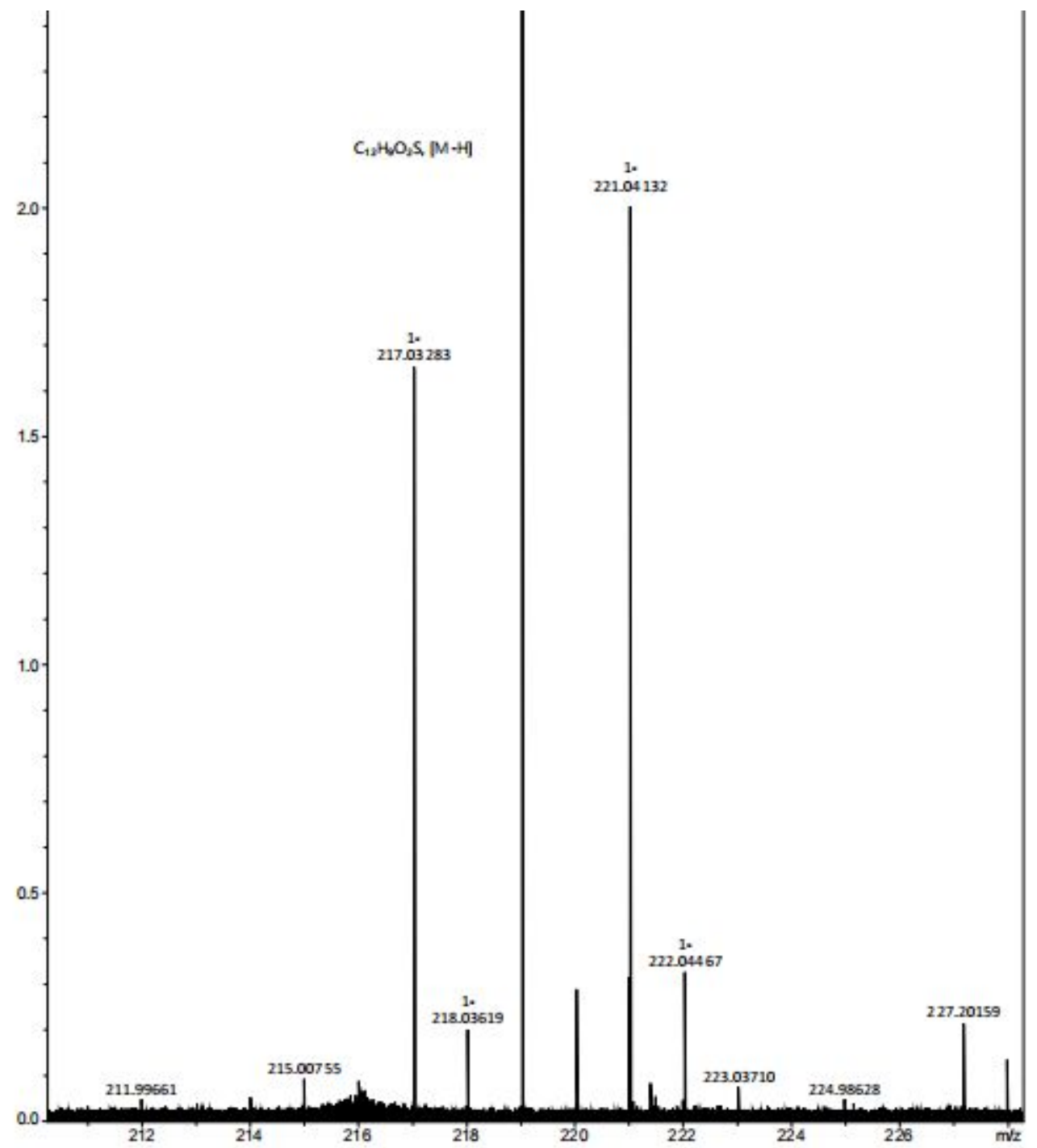

Figure S1. HRMS of ${ }^{18} \mathrm{O}-2 \mathrm{a}$

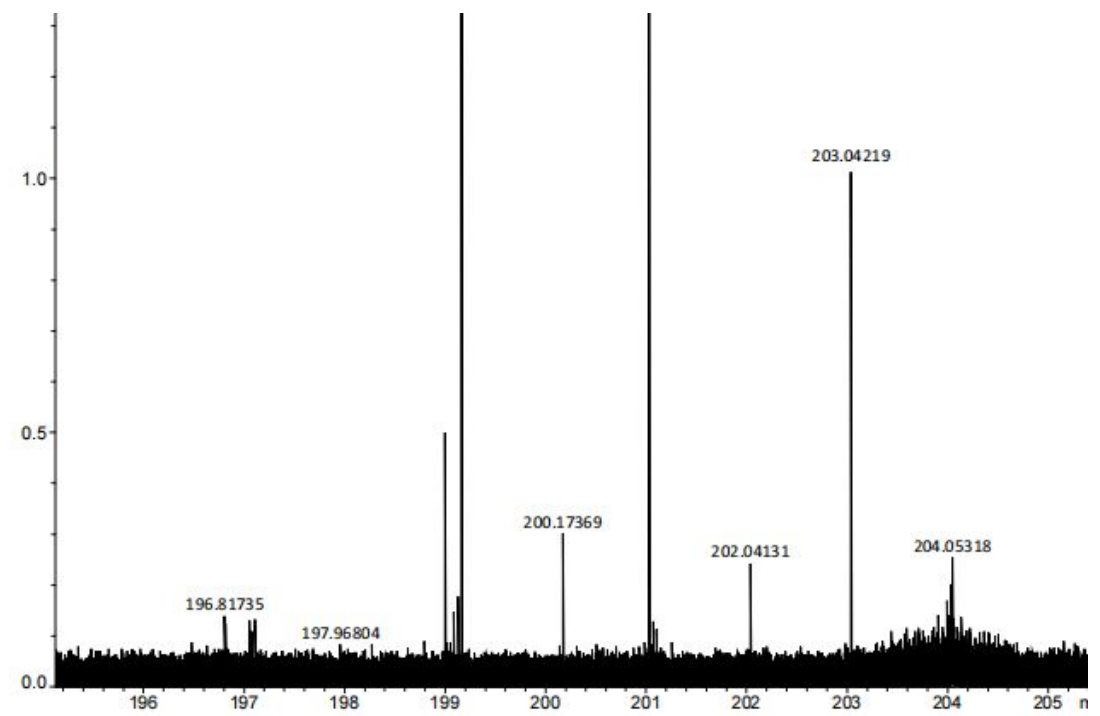

Figure S2. HRMS of ${ }^{18} \mathrm{O}-3 \mathrm{a}$ 


\subsubsection{Isotope labeling experiment with $\mathrm{H}_{2}{ }^{18} \mathrm{O}$}

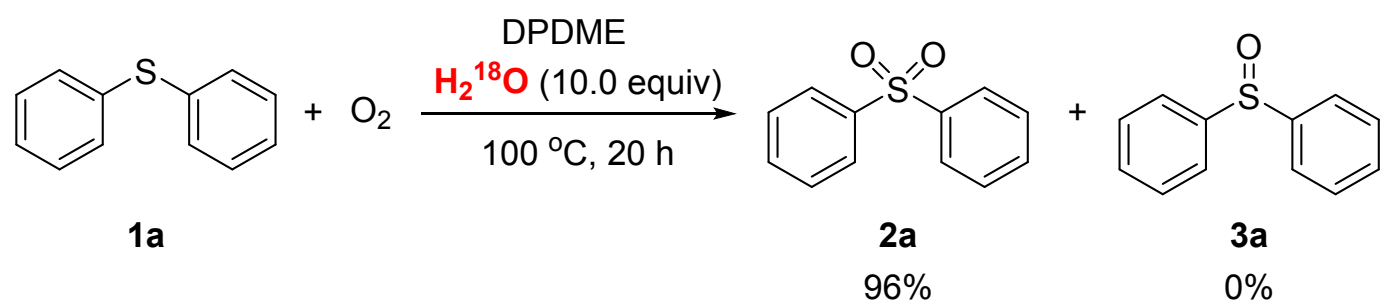

A mixture of diphenyl sulfide $1 \mathrm{a}(93 \mathrm{mg}, 0.50 \mathrm{mmol})$, DPDME $(0.5 \mathrm{~mL})$ and $\mathrm{H}_{2}{ }^{18} \mathrm{O}(100 \mathrm{mg}, 5.0 \mathrm{mmol})$ were added to a $10 \mathrm{~mL}$ Schlenk tube. The reaction tube was flushed with $\mathrm{O}_{2}$ for 1.0 minute and then equipped with a $\mathrm{O}_{2}$ balloon. The reaction was stirred at $100{ }^{\circ} \mathrm{C}$ for $20 \mathrm{~h}$. After cooling to ambient temperature, the resulting mixture was extracted with EtOAc $(3 \times 10 \mathrm{~mL})$. The combined organic phase was dried over anhydrous $\mathrm{MgSO}_{4}$, filtered, and all the volatiles were evaporated under reduced pressure. The resultant residue was purified by silica gel column chromatography (eluent: petroleum ether $\left.\left(60-90{ }^{\circ} \mathrm{C}\right) / \mathrm{EtOAc}=25: 1, \mathrm{v} / \mathrm{v}\right)$ to afford the desired product 2a in $96 \%$ yield. Then the product was analyzed by HRMS. HRMS of 2a: calcd for $\mathrm{C}_{12} \mathrm{H}_{10} \mathrm{O}_{2} \mathrm{SNa}[\mathrm{M}+\mathrm{Na}]^{+}$: 241.0299; found 241.0298.

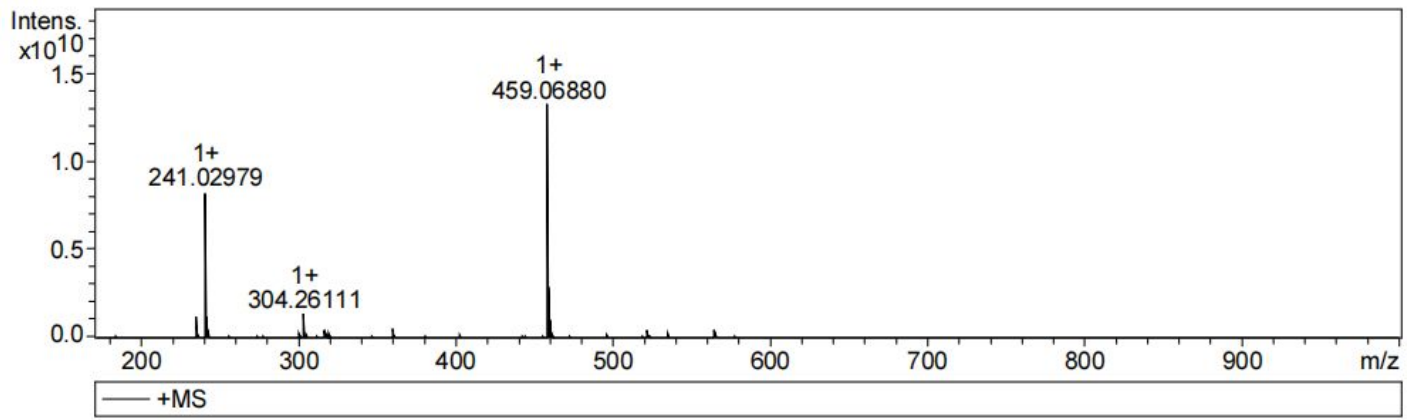

Figure S3. HRMS of 2a

\subsection{Intermediate probe experiment}

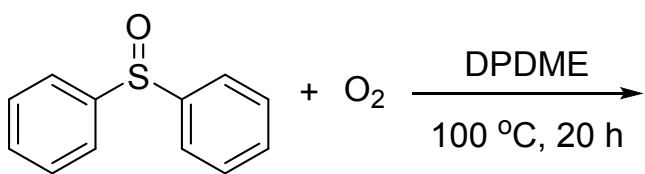

3a<smiles>O=S(=O)(c1ccccc1)c1ccccc1</smiles>

$2 a$

$95 \%$

A mixture of diphenyl sulfoxide 3a (101 $\mathrm{mg}, 0.50 \mathrm{mmol})$ and DPDME $(0.5 \mathrm{~mL})$ were added to a $10 \mathrm{~mL}$ Schlenk tube. The reaction tube was flushed with $\mathrm{O}_{2}$ for 1.0 minute and then equipped with a $\mathrm{O}_{2}$ balloon. The reaction was stirred at $100{ }^{\circ} \mathrm{C}$ for 20 
h. After cooling to ambient temperature, the resulting mixture was extracted with EtOAc $(3 \times 10 \mathrm{~mL})$. The combined organic phase was dried over anhydrous $\mathrm{MgSO}_{4}$, filtered, and all the volatiles were evaporated under reduced pressure. The resultant residue was purified by silica gel column chromatography (eluent: petroleum ether $\left.\left(60-90{ }^{\circ} \mathrm{C}\right) / \mathrm{EtOAc}=25: 1, \mathrm{v} / \mathrm{v}\right)$ to afford the desired product $\mathbf{2 a}$ in $95 \%$ yield.

\subsection{Eelectron paramagnetic resonance (EPR) experiment}

A mixture of diphenyl sulfide $1 \mathrm{a}(93 \mathrm{mg}, 0.50 \mathrm{mmol})$ and DPDME $(0.5 \mathrm{~mL})$ were added to a $10 \mathrm{~mL}$ reaction tube. The reaction was stirred under air at $80{ }^{\circ} \mathrm{C}$ for $20 \mathrm{~min}$, followed by the addition of DMPO (5,5-dimethyl-1-pyrroline $N$-oxide). Then, this mixture was analyzed by EPR.

Measurement conditions: Power 2mW, Frequency $9.054 \mathrm{GHz}$, Center field $323.100 \mathrm{mT}$, Sweep width $10 \mathrm{mT}$, Modulation width $0.2 \mathrm{mT}$, Sweep time $1.0 \mathrm{~min}$, Time constant $0.03 \mathrm{~s}$.

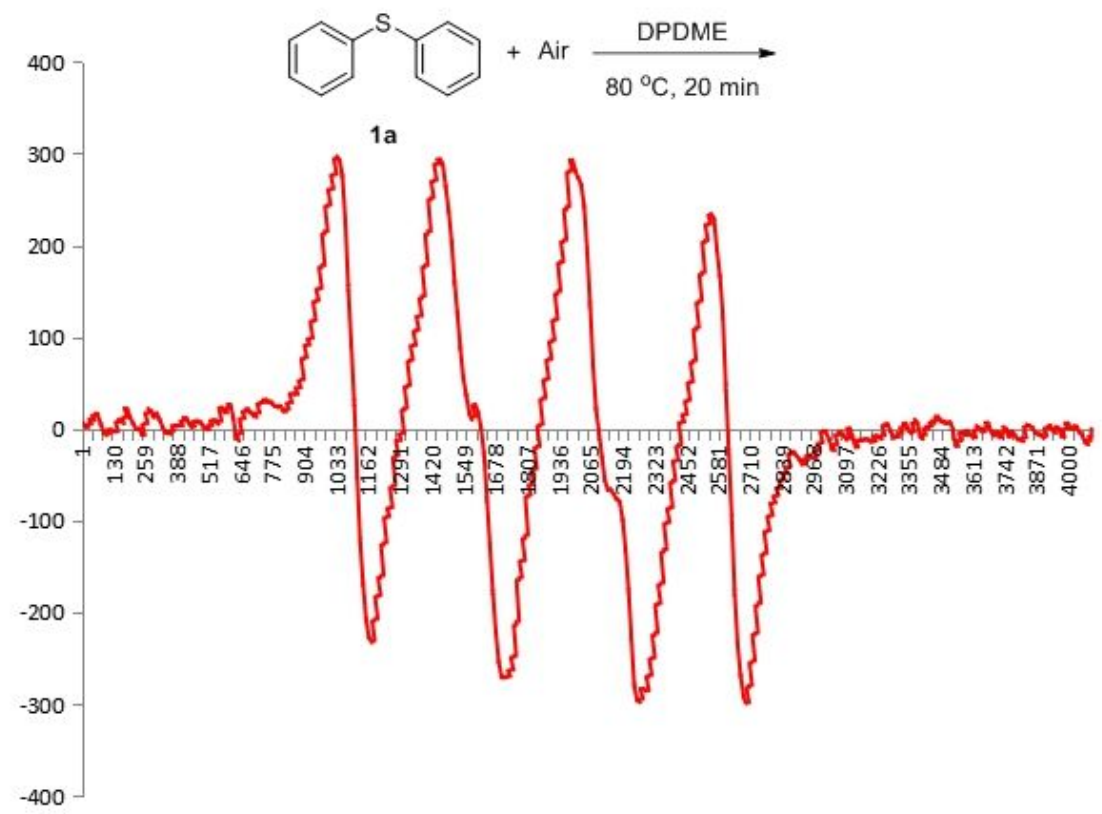

Figure S4. Eelectron Paramagnetic Resonance (EPR) Spectra (X Band, 9.054 GHz, Room Temperature)

\subsection{In-situ IR experiment}


+ Air $\frac{100^{\circ} \mathrm{C}, 12 \mathrm{~h}}{\text { DPDME }}$

$1 \mathrm{a}$<smiles>O=S(=O)(c1ccccc1)c1ccccc1</smiles>

$2 a$<smiles>O=S(c1ccccc1)c1ccccc1</smiles>

3a

A mixture of diphenyl sulfide 1a (559 mg, $3 \mathrm{mmol})$ and DPDME (10 mL) were added to a $50 \mathrm{~mL}$ three-necked flask. The mixture was allowed to stir at $100{ }^{\circ} \mathrm{C}$ for 12 $\mathrm{h}$ and then recorded by in-situ IR.

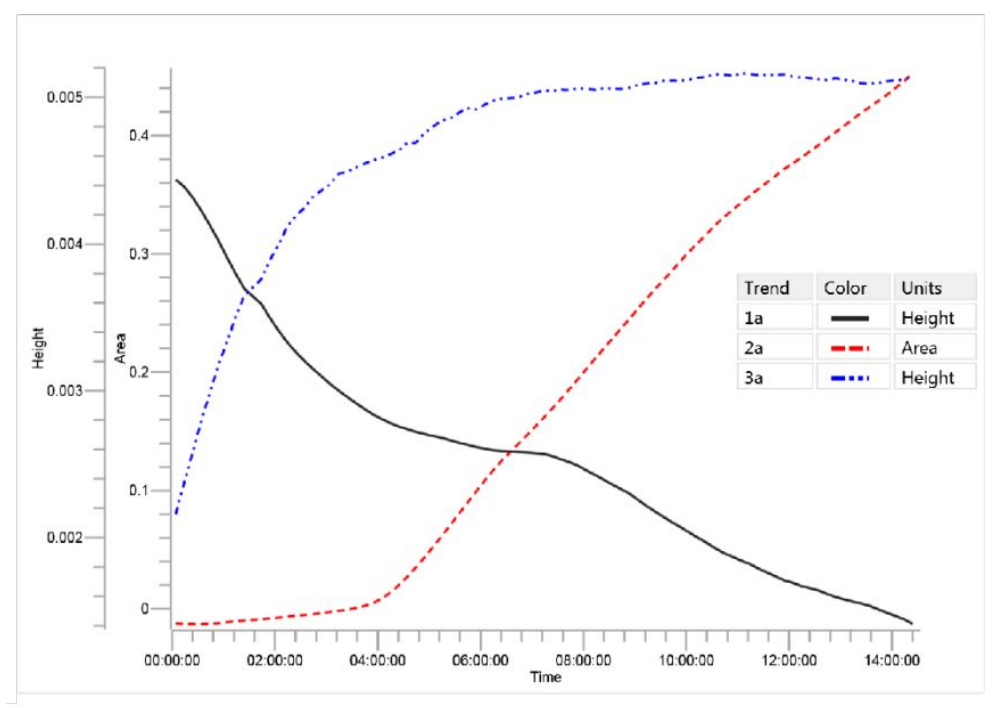

Figure S5. Trend Chart of Concentration Change with Time for 1a, 2a and 3a

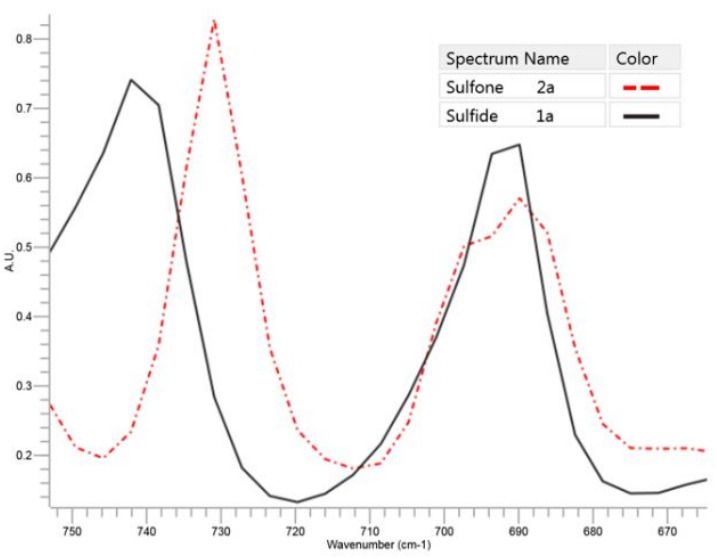

Figure S6. The Characteristic IR Bands of 1a and 2a

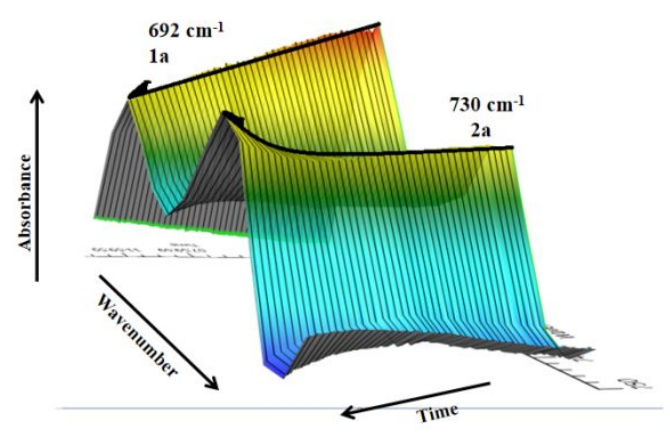

Figure S7. 3D Kinetic Plots of Sulfide 1a and Sulfone 2a 


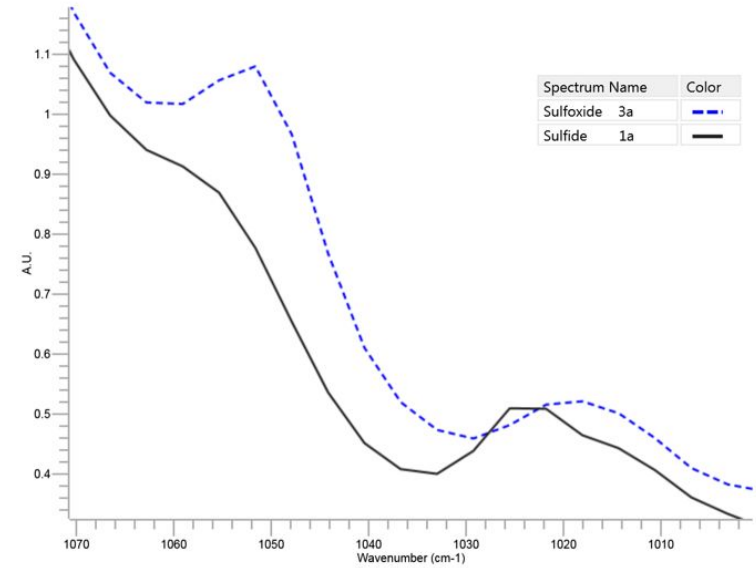

Figure S8. The Characteristic IR Bands of $1 \mathrm{a}$ and $3 \mathrm{a}$

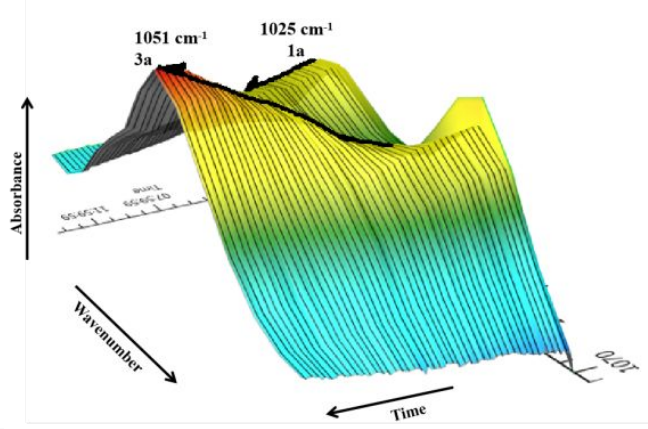

Figure S9. 3D Kinetic Plots of Sulfide 1a and Sulfoxide 3a

\section{Analytical data}<smiles>O=S(=O)(c1ccccc1)c1ccccc1</smiles>

Sulfonyldibenzene (2a) ${ }^{3}$ : Known compound. Flash chromatography (200-300 mesh silica gel, eluent: petroleum ether $\left.\left(60-90{ }^{\circ} \mathrm{C}\right) / \mathrm{EtOAc}=25: 1, \mathrm{v} / \mathrm{v}\right) .101 \mathrm{mg}, 93 \%$ yield. White solid. m.p.: 69.0-70.0 ${ }^{\circ} \mathrm{C} .{ }^{1} \mathbf{H}$ NMR $\left(\mathrm{CDCl}_{3}, 400 \mathrm{MHz}\right) \delta 7.94(\mathrm{~d}, J=7.0$ $\mathrm{Hz}, 4 \mathrm{H})$, 7.57-7.53 (m, 2H), 7.51-7.47 (m, 4H); ${ }^{13} \mathbf{C ~ N M R ~}\left(\mathrm{CDCl}_{3}, 100 \mathrm{MHz}\right) \delta 141.7$, $133.3,129.4,127.7$.<smiles>O=S(=O)(c1ccccc1)c1ccc(Br)cc1</smiles>

1-Bromo-4-(phenylsulfonyl)benzene $\quad(\mathbf{2 b})^{3}$ : Known compound. Flash chromatography (200-300 mesh silica gel, eluent: petroleum ether $\left(60-90{ }^{\circ} \mathrm{C}\right) / \mathrm{EtOAc}$ $=25: 1, \mathrm{v} / \mathrm{v}) .117 \mathrm{mg}, 79 \%$ yield. White solid. m.p.: 105.0-108.0 ${ }^{\circ} \mathrm{C} .{ }^{1} \mathbf{H} \mathbf{N M R}\left(\mathrm{CDCl}_{3}\right.$, $400 \mathrm{MHz}) \delta 7.92(\mathrm{~d}, J=7.4 \mathrm{~Hz}, 2 \mathrm{H}), 7.80(\mathrm{~d}, J=8.4 \mathrm{~Hz}, 2 \mathrm{H}), 7.64-7.51(\mathrm{~m}, 5 \mathrm{H}) ;{ }^{13} \mathrm{C}$ NMR $\left(\mathrm{CDCl}_{3}, 100 \mathrm{MHz}\right) \delta 141.2,140.7,133.6,132.7,129.5,129.3,128.5,127.7$.<smiles>O=S(=O)(c1ccc(Br)cc1)c1ccc(Br)cc1</smiles>

4,4'-Sulfonylbis(bromobenzene) $\quad(\mathbf{2 c})^{3}$ : Known compound. Flash 
chromatography (200-300 mesh silica gel, eluent: petroleum ether $\left(60-90{ }^{\circ} \mathrm{C}\right) / \mathrm{EtOAc}$ $=25: 1, \mathrm{v} / \mathrm{v}) .152 \mathrm{mg}, 81 \%$ yield. White solid. m.p.: 168.0-169.0 ${ }^{\circ} \mathrm{C} .{ }^{1} \mathbf{H}$ NMR $\left(\mathrm{CDCl}_{3}\right.$, $400 \mathrm{MHz}) \delta$ 7.80-7.76 (m, 4H), 7.67-7.63 (m, 4H); ${ }^{13} \mathbf{C}$ NMR $\left(\mathrm{CDCl}_{3}, 100\right.$ MHz) $\delta 140.4,132.9,129.3,128.9$.<smiles>CS(=O)(=O)c1ccccc1</smiles>

(Methylsulfonyl)benzene (2d) ${ }^{4}$ : Known compound. Flash chromatography (200-300 mesh silica gel, eluent: petroleum ether $\left.\left(60-90{ }^{\circ} \mathrm{C}\right) / \mathrm{EtOAc}=25: 1, \mathrm{v} / \mathrm{v}\right) .75$ mg, 96\% yield. Colorless oil. ${ }^{1} \mathbf{H}$ NMR $\left(\mathrm{CDCl}_{3}, 400 \mathrm{MHz}\right) \delta 7.91-7.89(\mathrm{~m}, 2 \mathrm{H})$, 7.62-7.51 (m, 3H), $3.01(\mathrm{~s}, 3 \mathrm{H}) ;{ }^{13} \mathbf{C} \mathbf{N M R}\left(\mathrm{CDCl}_{3}, 100 \mathrm{MHz}\right) \delta 140.5,133.7,129.4$, $127.3,44.4$.<smiles>COc1ccc(S(C)(=O)=O)cc1</smiles>

1-Methoxy-4-(methylsulfonyl)benzene (2e) ${ }^{4}$ : Known compound. Flash chromatography (200-300 mesh silica gel, eluent: petroleum ether $\left(60-90{ }^{\circ} \mathrm{C}\right) / \mathrm{EtOAc}$ $=5: 1, \mathrm{v} / \mathrm{v}) .87 \mathrm{mg}, 93 \%$ yield. White solid. m.p.: 120.0-122.0 ${ }^{\circ} \mathrm{C} .{ }^{1} \mathbf{H} \mathbf{~ N M R}\left(\mathrm{CDCl}_{3}\right.$, $400 \mathrm{MHz}) \delta 7.84(\mathrm{~d}, J=8.6 \mathrm{~Hz}, 2 \mathrm{H}), 7.00(\mathrm{~d}, J=8.7 \mathrm{~Hz}, 2 \mathrm{H}), 3.86$ (s, 3H), 3.01(s, $3 \mathrm{H}) ;{ }^{13} \mathbf{C}$ NMR $\left(\mathrm{CDCl}_{3}, 100 \mathrm{MHz}\right) \delta 163.8,132.3,129.6,114.6,55.8,44.9$.<smiles>CS(=O)(=O)c1ccccc1F</smiles>

1-Fluoro-2-(methylsulfonyl)benzene $\quad \mathbf{( 2 f}^{5}$ : Known compound. Flash chromatography (200-300 mesh silica gel, eluent: petroleum ether $\left(60-90^{\circ} \mathrm{C}\right) / \mathrm{EtOAc}$ $=5: 1, \mathrm{v} / \mathrm{v}) .68 \mathrm{mg}, 78 \%$ yield. White solid. m.p.: 48.0-50.0 ${ }^{\circ} \mathrm{C} .{ }^{1} \mathbf{H}$ NMR $\left(\mathrm{CDCl}_{3}, 400\right.$ MHz) $\delta$ 7.92-7.88 (m, 1H), 7.63-7.61 (m, 1H), 7.32-7.19 (m, 2H), $3.18(\mathrm{~s}, 3 \mathrm{H}) ;{ }^{13} \mathrm{C}$ NMR $\left(\mathrm{CDCl}_{3}, 100 \mathrm{MHz}\right) \delta 159.4(\mathrm{~d}, J=254.0 \mathrm{~Hz}), 136.2(\mathrm{~d}, J=9.0 \mathrm{~Hz}), 129.6$, $128.3(\mathrm{~d}, J=15.0 \mathrm{~Hz}), 124.8(\mathrm{~d}, J=4.0 \mathrm{~Hz}), 117.2(\mathrm{~d}, J=21.0 \mathrm{~Hz}), 43.8(\mathrm{~d}, J=3.0$ $\mathrm{Hz})$. 
<smiles>CS(=O)(=O)c1cccc(F)c1</smiles>

1-Fluoro-3-(methylsulfonyl)benzene $\quad \mathbf{( 2 g}^{6}$ : Known compound. Flash chromatography (200-300 mesh silica gel, eluent: petroleum ether $\left(60-90{ }^{\circ} \mathrm{C}\right) / \mathrm{EtOAc}$ $=5: 1, \mathrm{v} / \mathrm{v}) .80 \mathrm{mg}, 92 \%$ yield. Colorless oil. ${ }^{1} \mathbf{H} \mathbf{~ N M R}\left(\mathrm{CDCl}_{3}, 400 \mathrm{MHz}\right) \delta 7.78-7.75$ (m, 1H), 7.67-7.60 (m, 2H), 7.39-7.38 (m, 1H), $3.10(\mathrm{~s}, 3 \mathrm{H}) ;{ }^{13} \mathbf{C ~ N M R}\left(\mathrm{CDCl}_{3}, 100\right.$ MHz) $\delta 162.3(\mathrm{~d}, J=250.0 \mathrm{~Hz}), 142.4(\mathrm{~d}, J=7.0 \mathrm{~Hz}), 131.3(\mathrm{~d}, J=8.0 \mathrm{~Hz}), 123.1$, $120.9(\mathrm{~d}, J=22.0 \mathrm{~Hz}), 114.6(\mathrm{~d}, J=25.0 \mathrm{~Hz}), 44.2$.<smiles>CS(=O)(=O)c1ccc(F)cc1</smiles>

1-Fluoro-4-(methylsulfonyl)benzene $\quad(\mathbf{2 h})^{4}$ : Known compound. Flash chromatography (200-300 mesh silica gel, eluent: petroleum ether $\left(60-90{ }^{\circ} \mathrm{C}\right) / \mathrm{EtOAc}$ $=5: 1, \mathrm{v} / \mathrm{v}) .82 \mathrm{mg}, 94 \%$ yield. Colorless oil. ${ }^{1} \mathbf{H} \mathbf{N M R}\left(\mathrm{CDCl}_{3}, 400 \mathrm{MHz}\right) \delta$ 7.95-7.92 (m, 2H), 7.24-7.19 (m, 2H), $3.03(\mathrm{~s}, 3 \mathrm{H}) ;{ }^{13} \mathbf{C}$ NMR $\left(\mathrm{CDCl}_{3}, 100 \mathrm{MHz}\right) \delta 165.8(\mathrm{~d}, J$ $=254.0 \mathrm{~Hz}), 136.7(\mathrm{~d}, J=3.0 \mathrm{~Hz}), 130.3(\mathrm{~d}, J=10.0 \mathrm{~Hz}), 116.7(\mathrm{~d}, J=23.0 \mathrm{~Hz})$, 44.7.<smiles>CS(=O)(=O)c1ccccc1Cl</smiles>

1-Chloro-2-(methylsulfonyl)benzene $\quad \mathbf{( 2 i}^{4}$ : Known compound. Flash chromatography (200-300 mesh silica gel, eluent: petroleum ether $\left(60-90{ }^{\circ} \mathrm{C}\right) / \mathrm{EtOAc}$ $=1: 2, \mathrm{v} / \mathrm{v}) .90 \mathrm{mg}, 94 \%$ yield. White solid. m.p.: 80.0-82.0 ${ }^{\circ} \mathrm{C} .{ }^{1} \mathbf{H}$ NMR $\left(\mathrm{CDCl}_{3}, 400\right.$ $\mathrm{MHz}) \delta 8.12-8.09(\mathrm{~m}, 1 \mathrm{H}), 7.58-7.51(\mathrm{~m}, 2 \mathrm{H}), 7.47-7.42(\mathrm{~m}, 1 \mathrm{H}), 3.24(\mathrm{~s}, 3 \mathrm{H}) ;{ }^{13} \mathrm{C}$ NMR $\left(\mathrm{CDCl}_{3}, 100 \mathrm{MHz}\right) \delta 138.0,134.9,132.5,131.9,130.8,127.6,42.8$.<smiles>CS(=O)(=O)c1cccc(Cl)c1</smiles>

1-Chloro-3-(methylsulfonyl)benzene $\left.\quad \mathbf{( 2 j}^{7}\right)^{7}$ Known compound. Flash chromatography (200-300 mesh silica gel, eluent: petroleum ether $\left(60-90{ }^{\circ} \mathrm{C}\right) / \mathrm{EtOAc}$ 
$=5: 1, \mathrm{v} / \mathrm{v}) .91 \mathrm{mg}, 95 \%$ yield. White solid. m.p.: 56.0-58.0 ${ }^{\circ} \mathrm{C} .{ }^{1} \mathbf{H} \mathbf{~ N M R}\left(\mathrm{CDCl}_{3}, 400\right.$ MHz) $\delta 7.90(\mathrm{~s}, 1 \mathrm{H}), 7.80(\mathrm{~d}, J=7.8 \mathrm{~Hz}, 1 \mathrm{H}), 7.60(\mathrm{~d}, J=8.0 \mathrm{~Hz}, 1 \mathrm{H}), 7.50(\mathrm{t}, J=$ $7.9 \mathrm{~Hz}, 1 \mathrm{H}), 3.04(\mathrm{~s}, 3 \mathrm{H}) ;{ }^{13} \mathrm{C}$ NMR $\left(\mathrm{CDCl}_{3}, 100 \mathrm{MHz}\right) \delta 142.2,135.6,133.9,130.8$, $127.6,125.6,44.4$.<smiles>CS(=O)(=O)c1ccc(Cl)cc1</smiles>

1-Chloro-4-(methylsulfonyl)benzene $\quad(\mathbf{2 k})^{4}$ : Known compound. Flash chromatography (200-300 mesh silica gel, eluent: petroleum ether $\left(60-90{ }^{\circ} \mathrm{C}\right) / \mathrm{EtOAc}$ $=5: 1, \mathrm{v} / \mathrm{v}) .91 \mathrm{mg}, 95 \%$ yield. White solid. m.p.: 94.0-95.0 ${ }^{\circ} \mathrm{C} .{ }^{\mathbf{1}} \mathbf{H} \mathbf{~ N M R}\left(\mathrm{CDCl}_{3}, 400\right.$ MHz) $\delta 7.86(\mathrm{~d}, J=8.5 \mathrm{~Hz}, 2 \mathrm{H}), 7.53(\mathrm{~d}, J=8.4 \mathrm{~Hz}, 2 \mathrm{H}), 3.03(\mathrm{~s}, 3 \mathrm{H}) ;{ }^{13} \mathbf{C}$ NMR $\left(\mathrm{CDCl}_{3}, 100 \mathrm{MHz}\right) \delta 140.5,139.1,129.8,129.0,44.6$.<smiles>CS(=O)(=O)c1ccccc1Br</smiles>

1-Bromo-2-(methylsulfonyl)benzene $\quad \mathbf{( 2 l}^{8}$ : Known compound. Flash chromatography (200-300 mesh silica gel, eluent: petroleum ether $\left(60-90{ }^{\circ} \mathrm{C}\right) / \mathrm{EtOAc}$ $=1: 1, \mathrm{v} / \mathrm{v}) .88 \mathrm{mg}, 75 \%$ yield. White solid. m.p.: 105.0-106.0 ${ }^{\circ} \mathrm{C} .{ }^{1} \mathbf{H}$ NMR $\left(\mathrm{CDCl}_{3}\right.$, $400 \mathrm{MHz}) \delta 8.16(\mathrm{~d}, J=7.4 \mathrm{~Hz}, 1 \mathrm{H}), 7.75(\mathrm{~d}, \mathrm{~J}=7.4 \mathrm{~Hz}, 1 \mathrm{H}), 7.53-7.44(\mathrm{~m}, 2 \mathrm{H})$, $3.26(\mathrm{~s}, 3 \mathrm{H}) ;{ }^{13} \mathrm{C}$ NMR $\left(\mathrm{CDCl}_{3}, 100 \mathrm{MHz}\right) \delta 139.6,135.4,134.8,131.1,128.2,120.6$, 42.5 .<smiles>CS(=O)(=O)c1ccc(Br)cc1</smiles>

1-Bromo-4-(methylsulfonyl)benzene $\quad(\mathbf{2 m})^{4}$ : Known compound. Flash chromatography (200-300 mesh silica gel, eluent: petroleum ether $\left(60-90{ }^{\circ} \mathrm{C}\right) / \mathrm{EtOAc}$ $=1: 2, \mathrm{v} / \mathrm{v}) .115 \mathrm{mg}, 98 \%$ yield. White solid. m.p.: $100.0-102.0^{\circ} \mathrm{C} .{ }^{1} \mathbf{H}$ NMR $\left(\mathrm{CDCl}_{3}\right.$, $400 \mathrm{MHz}) \delta$ 7.78-7.76 (m, 2H), 7.69-7.67 (m, 2H), $3.02(\mathrm{~s}, 3 \mathrm{H}) ;{ }^{13} \mathbf{C}$ NMR $\left(\mathrm{CDCl}_{3}\right.$, $100 \mathrm{MHz}) \delta 139.6,132.7,129.0,128.9,44.5$. 
<smiles>CS(=O)(=O)c1ccc(C(=O)O)cc1</smiles>

2-(Methylsulfonyl)benzoic acid (2n) ${ }^{9}$ : Known compound. Flash chromatography (200-300 mesh silica gel, eluent: EtOAc/MeOH = 3:1, v/v). $87 \mathrm{mg}$, $87 \%$ yield. Yellow solid. m.p.: 245.0-250.0 ${ }^{\circ} \mathrm{C} .{ }^{1} \mathbf{H}$ NMR (DMSO- $d_{6}, 400$ MHz) $\delta 13.48(\mathrm{~s}, 1 \mathrm{H}), 8.18-8.16(\mathrm{~m}, 2 \mathrm{H}), 8.06-8.04(\mathrm{~m}, 2 \mathrm{H}), 3.28(\mathrm{~s}, 3 \mathrm{H}) ;{ }^{13} \mathbf{C}$ NMR $\left(\mathrm{DMSO}-d_{6}, 100 \mathrm{MHz}\right) \delta 166.2,144.3,135.2,130.2,127.3,43.2$.<smiles>CC(=O)c1ccc(S(C)(=O)=O)cc1</smiles>

1-(4-(Methylsulfonyl)phenyl)ethan-1-one $(\mathbf{2 o})^{6}$ : Known compound. Flash chromatography (200-300 mesh silica gel, eluent: petroleum ether $\left(60-90^{\circ} \mathrm{C}\right) / \mathrm{EtOAc}$ $=1: 1, \mathrm{v} / \mathrm{v}) .71 \mathrm{mg}, 72 \%$ yield. White solid. m.p.: 120.0-122.0 ${ }^{\circ} \mathrm{C} .{ }^{\mathbf{1}} \mathbf{H} \mathbf{~ N M R}\left(\mathrm{CDCl}_{3}\right.$, $400 \mathrm{MHz}) \delta 8.08(\mathrm{~d}, J=8.2 \mathrm{~Hz}, 2 \mathrm{H}), 7.99$ (d, $J=8.2 \mathrm{~Hz}, 2 \mathrm{H}), 3.05$ (s, 3H), 2.62 (s, 3H); ${ }^{13} \mathrm{C}$ NMR $\left(\mathrm{CDCl}_{3}, 100 \mathrm{MHz}\right) \delta 196.7,144.1,140.8,129.1,127.7,44.2,26.9$.<smiles>O=S(=O)(c1ccccc1)C1CC1</smiles>

(Cyclopropylsulfonyl)benzene (2p) ${ }^{4}$ : Known compound. Flash chromatography (200-300 mesh silica gel, eluent: petroleum ether $\left.\left(60-90{ }^{\circ} \mathrm{C}\right) / \mathrm{EtOAc}=2: 1, \mathrm{v} / \mathrm{v}\right) .88$ mg, 96\% yield. colorless oil. ${ }^{1} \mathbf{H}$ NMR $\left(\mathrm{CDCl}_{3}, 400 \mathrm{MHz}\right) \delta 7.87-7.84(\mathrm{~m}, 2 \mathrm{H})$, 7.62-7.58 (m, 1H), 7.54-7.50 (m, 2H), 2.47-2.40 (m, 1H), 1.31-1.27 (m, 2H), 1.02-0.96 (m, 2H); ${ }^{13} \mathrm{C}$ NMR $\left(\mathrm{CDCl}_{3}, 100 \mathrm{MHz}\right) \delta 140.7,133.4,129.2,127.5,32.9$, 6.0 .<smiles>O=S(=O)(Cc1ccccc1)c1ccccc1</smiles>

(Benzylsulfonyl)benzene (2q) $)^{4}$ Known compound. Flash chromatography (200-300 mesh silica gel, eluent: petroleum ether $\left.\left(60-90{ }^{\circ} \mathrm{C}\right) / \mathrm{EtOAc}=2: 1, \mathrm{v} / \mathrm{v}\right) .91$ 
mg, 78\% yield. White solid. m.p.: 148.0-149.0 ${ }^{\circ} \mathrm{C} .{ }^{1} \mathbf{H} \mathbf{N M R}\left(\mathrm{CDCl}_{3}, 400\right.$ MHz) $\delta$ 7.64-7.58 (m, 3H), $7.45(\mathrm{t}, J=7.6 \mathrm{~Hz}, 2 \mathrm{H}), 7.34-7.24(\mathrm{~m}, 3 \mathrm{H}), 7.08$ (d, $J=$ $7.3 \mathrm{~Hz}, 2 \mathrm{H}), 4.31(\mathrm{~s}, 2 \mathrm{H}) ;{ }^{13} \mathrm{C}$ NMR $\left(\mathrm{CDCl}_{3}, 100 \mathrm{MHz}\right) \delta 137.9,133.8,130.9,129.0$, $128.9,128.8,128.7,128.2,63.0$.<smiles>CS(=O)(=O)c1ccccn1</smiles>

2-(Methylsulfonyl)pyridine (2r) ${ }^{5}$ : Known compound. Flash chromatography (200-300 mesh silica gel, eluent: petroleum ether $\left.\left(60-90{ }^{\circ} \mathrm{C}\right) / \mathrm{EtOAc}=1: 1, \mathrm{v} / \mathrm{v}\right) .34$ mg, $43 \%$ yield. Yellow oil. ${ }^{1} \mathbf{H}$ NMR $\left(\mathrm{CDCl}_{3}, 400 \mathrm{MHz}\right) \delta 8.73(\mathrm{~d}, J=4.6 \mathrm{~Hz}, 1 \mathrm{H})$, 8.09-7.95 (m, 2H), 7.57-7.54 (m, 1H), $3.22(\mathrm{~s}, 3 \mathrm{H}) ;{ }^{13} \mathbf{C}$ NMR $\left(\mathrm{CDCl}_{3}, 100\right.$ MHz) $\delta 158.1,150.2,138.4,127.6,121.2,40.1$.<smiles>CS(=O)(=O)c1nc(Cl)cc(Cl)n1</smiles>

4,6-Dichloro-2-(methylsulfonyl)pyrimidine $(2 s)^{10}$ : Known compound. Flash chromatography (200-300 mesh silica gel, eluent: petroleum ether $\left(60-90{ }^{\circ} \mathrm{C}\right) / \mathrm{EtOAc}$ $=1: 1, \mathrm{v} / \mathrm{v}) .27 \mathrm{mg}, 24 \%$ yield. White solid. m.p.: 112.0-114.0 ${ }^{\circ} \mathrm{C} .{ }^{\mathbf{1}} \mathbf{H}$ NMR $\left(\mathrm{CDCl}_{3}\right.$, $400 \mathrm{MHz}) \delta 7.62(\mathrm{~s}, 1 \mathrm{H}), 3.38(\mathrm{~s}, 3 \mathrm{H}) ;{ }^{13} \mathrm{C}$ NMR $\left(\mathrm{CDCl}_{3}, 100 \mathrm{MHz}\right) \delta$ 166.1, 164.0, 124.6, 39.3.<smiles>O=S(c1ccccc1)c1ccccc1</smiles>

Sulfinyldibenzene (3a) ${ }^{11}$ : Known compound. Flash chromatography (200-300 mesh silica gel, eluent: petroleum ether $\left.\left(60-90{ }^{\circ} \mathrm{C}\right) / \mathrm{EtOAc}=2: 1, \mathrm{v} / \mathrm{v}\right) .74 \mathrm{mg}, 72 \%$ yield. White solid. m.p.: $60.0-61.0{ }^{\circ} \mathrm{C} .{ }^{1} \mathbf{H}$ NMR $\left(\mathrm{CDCl}_{3}, 400 \mathrm{MHz}\right) \delta 7.65-7.63(\mathrm{~m}$, 4H), 7.47-7.42 (m, 6H); ${ }^{13} \mathbf{C}$ NMR $\left(\mathrm{CDCl}_{3}, 100 \mathrm{MHz}\right) \delta 145.7,131.2,129.4,124.9$.<smiles>O=S(c1ccccc1)c1ccc(Br)cc1</smiles> 
1-Bromo-4-(phenylsulfinyl)benzene $\quad(\mathbf{3 b})^{11}$ : Known compound. Flash chromatography (200-300 mesh silica gel, eluent: petroleum ether $\left(60-90{ }^{\circ} \mathrm{C}\right) / \mathrm{EtOAc}$ $=5: 1, \mathrm{v} / \mathrm{v}) .58 \mathrm{mg}, 41 \%$ yield. White solid. m.p.: 124.0-126.0 ${ }^{\circ} \mathrm{C} .{ }^{1} \mathbf{H}$ NMR $\left(\mathrm{CDCl}_{3}\right.$, $400 \mathrm{MHz}) \delta 7.64-7.62(\mathrm{~m}, 2 \mathrm{H}), 7.59(\mathrm{~d}, J=8.5 \mathrm{~Hz}, 2 \mathrm{H}), 7.51(\mathrm{~d}, J=8.5 \mathrm{~Hz}, 2 \mathrm{H})$,

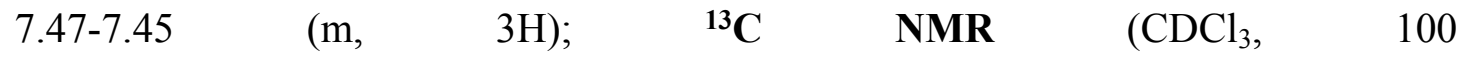
MHz) $\delta 145.3,145.0,132.6,131.5,129.6,126.3,125.7,124.8$.<smiles>O=S(c1ccc(Br)cc1)c1ccc(Br)cc1</smiles>

4,4'-Sulfinylbis(bromobenzene) (3c) ${ }^{12}: \quad$ Known $\quad$ compound. Flash chromatography (200-300 mesh silica gel, eluent: petroleum ether $\left(60-90{ }^{\circ} \mathrm{C}\right) / \mathrm{EtOAc}$ $=20: 1, \mathrm{v} / \mathrm{v}) .157 \mathrm{mg}, 87 \%$ yield. White solid. m.p.: 151.0-152.0 ${ }^{\circ} \mathrm{C} .{ }^{1} \mathbf{H}$ NMR $\left(\mathrm{CDCl}_{3}\right.$, $400 \mathrm{MHz}) \delta 7.60-7.56(\mathrm{~m}, 4 \mathrm{H}), 7.50-7.47 \quad(\mathrm{~m}, 4 \mathrm{H}) ;{ }^{13} \mathbf{C} \quad \mathbf{N M R}\left(\mathrm{CDCl}_{3}, 100\right.$ $\mathrm{MHz}) \delta 144.4,132.7,126.2,126.0$.<smiles>CS(=O)c1ccccc1</smiles>

(Methylsulfinyl)benzene (3d) ${ }^{13}$ : Known compound. Flash chromatography (200-300 mesh silica gel, eluent: petroleum ether $\left.\left(60-90{ }^{\circ} \mathrm{C}\right) / \mathrm{EtOAc}=1: 2, \mathrm{v} / \mathrm{v}\right) .12$ mg, $17 \%$ yield. Colorless oil. ${ }^{1} \mathbf{H}$ NMR $\left(\mathrm{CDCl}_{3}, 400 \mathrm{MHz}\right) \delta 7.63-7.61(\mathrm{~m}, 2 \mathrm{H})$, 7.52-7.46 (m, 3H), 2.69 (s, 3H); ${ }^{13} \mathbf{C}$ NMR $\left(\mathrm{CDCl}_{3}, 100 \mathrm{MHz}\right) \delta 145.7,131.1,129.4$, $123.5,44.0$.<smiles>CS(=O)c1ccccc1F</smiles>

1-Fluoro-2-(methylsulfinyl)benzene $\quad(3 e)^{14}$ : Known compound. Flash chromatography (200-300 mesh silica gel, eluent: petroleum ether $\left(60-90{ }^{\circ} \mathrm{C}\right) / \mathrm{EtOAc}$ $=1: 1, \mathrm{v} / \mathrm{v}) .53 \mathrm{mg}, 67 \%$ yield. Colorless oil. ${ }^{1} \mathbf{H} \mathbf{N M R}\left(\mathrm{CDCl}_{3}, 400 \mathrm{MHz}\right) \delta 7.87-7.83$ (m, 1H), 7.50-7.45 (m, 1H), $7.38(\mathrm{t}, J=7.3 \mathrm{~Hz}, 1 \mathrm{H}), 7.11(\mathrm{t}, J=9.3 \mathrm{~Hz}, 1 \mathrm{H}), 2.82(\mathrm{~s}$, $3 \mathrm{H}) ;{ }^{13} \mathrm{C}$ NMR $\left(\mathrm{CDCl}_{3}, 100 \mathrm{MHz}\right) \delta 157.6(\mathrm{~d}, J=246.0 \mathrm{~Hz}), 132.8(\mathrm{~d}, J=8.0 \mathrm{~Hz})$, 
$125.5 \times 3,115.8(\mathrm{~d}, J=20.0 \mathrm{~Hz}), 42.4$.<smiles>CS(=O)c1cccc(F)c1</smiles>

1-Fluoro-3-(methylsulfinyl)benzene (3f) ${ }^{13}$ : Known compound. Flash chromatography (200-300 mesh silica gel, eluent: petroleum ether $\left(60-90{ }^{\circ} \mathrm{C}\right) / \mathrm{EtOAc}$ $=1: 1, \mathrm{v} / \mathrm{v}) .60 \mathrm{mg}, 76 \%$ yield. Colorless oil. ${ }^{1} \mathbf{H}$ NMR $\left(\mathrm{CDCl}_{3}, 400 \mathrm{MHz}\right) \delta 7.53-7.48$ (m, 1H), 7.42-7.37 (m, 2H), 7.20-7.16 (m, 1H), $2.73(\mathrm{~s}, 3 \mathrm{H}) ;{ }^{13} \mathbf{C} \mathbf{~ N M R}\left(\mathrm{CDCl}_{3}, 100\right.$ MHz) $\delta 163.2(\mathrm{~d}, J=251.0 \mathrm{~Hz}), 148.6(\mathrm{~d}, J=4.0 \mathrm{~Hz}), 131.2(\mathrm{~d}, J=8.0 \mathrm{~Hz}), 119.3(\mathrm{~d}$, $J=3.0 \mathrm{~Hz}), 118.3(\mathrm{~d}, J=21.0 \mathrm{~Hz}), 111.0(\mathrm{~d}, J=24.0 \mathrm{~Hz}), 44.3$.<smiles>CS(=O)c1ccc(F)cc1</smiles>

1-Fluoro-4-(methylsulfinyl)benzene $\quad(\mathbf{3 g})^{13}$ : Known compound. Flash chromatography (200-300 mesh silica gel, eluent: petroleum ether $\left(60-90^{\circ} \mathrm{C}\right) / \mathrm{EtOAc}$ $=1: 2, \mathrm{v} / \mathrm{v}) .43 \mathrm{mg}, 54 \%$ yield. Colorless oil. ${ }^{\mathbf{1}} \mathbf{H} \mathbf{N M R}\left(\mathrm{CDCl}_{3}, 400 \mathrm{MHz}\right) \delta$ 7.63-7.60 (m, 2H), 7.21-7.16 (m, 2H), $2.68(\mathrm{~s}, 3 \mathrm{H}) ;{ }^{13} \mathrm{C}$ NMR $\left(\mathrm{CDCl}_{3}, 100 \mathrm{MHz}\right) \delta 164.3(\mathrm{~d}, J$ $=250.0 \mathrm{~Hz}), 141.2,125.8(\mathrm{~d}, J=9.0 \mathrm{~Hz}), 116.7(\mathrm{~d}, J=23.0 \mathrm{~Hz}), 44.2$.<smiles>CS(=O)c1ccccc1Cl</smiles>

1-Chloro-2-(methylsulfinyl)benzene $\quad \mathbf{( 3 h}^{14}$ : Known compound. Flash chromatography (200-300 mesh silica gel, eluent: petroleum ether $\left(60-90{ }^{\circ} \mathrm{C}\right) / \mathrm{EtOAc}$ $=1: 1, \mathrm{v} / \mathrm{v}) .51 \mathrm{mg}, 58 \%$ yield. Yellow oil. ${ }^{1} \mathbf{H}$ NMR $\left(\mathrm{CDCl}_{3}, 400 \mathrm{MHz}\right) \delta 7.87(\mathrm{~d}, J=$ $7.6 \mathrm{~Hz}, 1 \mathrm{H}), 7.46(\mathrm{t}, J=7.4 \mathrm{~Hz}, 1 \mathrm{H}), 7.39-7.30(\mathrm{~m}, 2 \mathrm{H}), 2.74(\mathrm{~s}, 3 \mathrm{H}) ;{ }^{13} \mathbf{C}$ NMR $\left(\mathrm{CDCl}_{3}, 100 \mathrm{MHz}\right) \delta 143.5,131.9,129.6 \times 2,128.0,125.1,41.6$.<smiles>CS(=O)c1cccc(Cl)c1</smiles>

1-Chloro-3-(methylsulfinyl)benzene $\quad$ (3i) $^{14}$ : Known compound. Flash chromatography (200-300 mesh silica gel, eluent: petroleum ether $\left(60-90{ }^{\circ} \mathrm{C}\right) / \mathrm{EtOAc}$ 
$=1: 2, \mathrm{v} / \mathrm{v}) .47 \mathrm{mg}, 54 \%$ yield. Colorless oil. ${ }^{1} \mathbf{H}$ NMR $\left(\mathrm{CDCl}_{3}, 400 \mathrm{MHz}\right) \delta 7.63-7.62$ (m, 1H), 7.47-7.42 (m, 3H), $2.70(\mathrm{~s}, 3 \mathrm{H}) ;{ }^{13} \mathbf{C} \mathbf{N M R}\left(\mathrm{CDCl}_{3}, 100 \mathrm{MHz}\right) \delta$ 147.9, $135.7,131.2,130.6,123.7,121.7,44.1$.<smiles>CS(=O)c1ccc(Cl)cc1</smiles>

1-Chloro-4-(methylsulfinyl)benzene $\quad(\mathbf{3 j})^{13}$ : Known compound. Flash chromatography (200-300 mesh silica gel, eluent: petroleum ether $\left(60-90{ }^{\circ} \mathrm{C}\right) / \mathrm{EtOAc}$ $=1: 2, \mathrm{v} / \mathrm{v}) .70 \mathrm{mg}, 80 \%$ yield. Colorless oil. ${ }^{1} \mathbf{H}$ NMR $\left(\mathrm{CDCl}_{3}, 400 \mathrm{MHz}\right) \delta$ 7.54-7.51 $(\mathrm{m}, 2 \mathrm{H}), 7.45-7.42(\mathrm{~m}, 2 \mathrm{H}), 2.65(\mathrm{~s}, 3 \mathrm{H}) ;{ }^{13} \mathbf{C} \mathbf{N M R}\left(\mathrm{CDCl}_{3}, 100 \mathrm{MHz}\right) \delta$ 144.2, $137.1,129.6,124.9,44.0$.<smiles>CS(=O)c1cc(Cl)cc(Cl)c1</smiles>

1,3-Dichloro-5-(methylsulfinyl)benzene (3k) ${ }^{13}$ : Known compound. Flash chromatography (200-300 mesh silica gel, eluent: petroleum ether $\left(60-90{ }^{\circ} \mathrm{C}\right) / \mathrm{EtOAc}$ $=1: 1, \mathrm{v} / \mathrm{v}) .73 \mathrm{mg}, 70 \%$ yield. White solid. m.p.: 70.0-71.0 ${ }^{\circ} \mathrm{C} .{ }^{\mathbf{1}} \mathbf{H} \mathbf{~ N M R}\left(\mathrm{CDCl}_{3}, 400\right.$ MHz) $\delta 7.49(\mathrm{~d}, J=1.8 \mathrm{~Hz}, 2 \mathrm{H}), 7.45(\mathrm{t}, J=1.8 \mathrm{~Hz}, 1 \mathrm{H}), 2.74(\mathrm{~s}, 3 \mathrm{H}) ;{ }^{13} \mathbf{C}$ NMR $\left(\mathrm{CDCl}_{3}, 100 \mathrm{MHz}\right) \delta 149.5,136.4,131.2,122.0,44.2$.<smiles>CS(=O)c1ccccc1Br</smiles>

1-Bromo-2-(methylsulfinyl)benzene (3I) ${ }^{8}$ : Known compound. Flash chromatography (200-300 mesh silica gel, eluent: petroleum ether $\left(60-90{ }^{\circ} \mathrm{C}\right) / \mathrm{EtOAc}$ $=1: 1, \mathrm{v} / \mathrm{v}) .76 \mathrm{mg}, 69 \%$ yield. Colorless oil. ${ }^{1} \mathbf{H}$ NMR $\left(\mathrm{CDCl}_{3}, 400 \mathrm{MHz}\right) \delta 7.93-7.91$ (m, 1H), 7.58-7.53 (m, 2H), 7.37-7.33 (m, 1H), $2.80(\mathrm{~s}, 3 \mathrm{H}) ;{ }^{13} \mathbf{C} \mathbf{N M R}\left(\mathrm{CDCl}_{3}, 100\right.$ MHz) $\delta 145.5,133.0,132.3,128.8,125.7,118.4,42.0$.<smiles>CS(=O)c1ccc(Br)cc1</smiles> 
1-Bromo-4-(methylsulfinyl)benzene $\quad(\mathbf{3 m})^{13}$ : Known compound. Flash chromatography (200-300 mesh silica gel, eluent: petroleum ether $\left(60-90{ }^{\circ} \mathrm{C}\right) / \mathrm{EtOAc}$ $=1: 2, \mathrm{v} / \mathrm{v}) .73 \mathrm{mg}, 67 \%$ yield. White solid. m.p.: 84.0-85.0 ${ }^{\circ} \mathrm{C} .{ }^{1} \mathbf{H} \mathbf{~ N M R}\left(\mathrm{CDCl}_{3}, 400\right.$ MHz) $\delta 7.60(\mathrm{~d}, J=8.4 \mathrm{~Hz}, 2 \mathrm{H}), 7.46(\mathrm{~d}, J=8.4 \mathrm{~Hz}, 2 \mathrm{H}), 2.65(\mathrm{~s}, 3 \mathrm{H}) ;{ }^{13} \mathbf{C}$ NMR $\left(\mathrm{CDCl}_{3}, 100 \mathrm{MHz}\right) \delta 144.9,132.5,125.3,125.1,44.0$.<smiles>CS(=O)c1ccc([N+](=O)[O-])cc1</smiles>

1-(Methylsulfinyl)-4-nitrobenzene $\quad(\mathbf{3 n})^{13}$ : Known compound. Flash chromatography (200-300 mesh silica gel, eluent: petroleum ether $\left(60-90{ }^{\circ} \mathrm{C}\right) / \mathrm{EtOAc}$ $=1: 5, \mathrm{v} / \mathrm{v}) .25 \mathrm{mg}, 27 \%$ yield. White solid. m.p.: 152.0-153.0 ${ }^{\circ} \mathrm{C} .{ }^{\mathbf{1}} \mathbf{H} \mathbf{N M R}\left(\mathrm{CDCl}_{3}\right.$, $400 \mathrm{MHz}) \delta 8.38(\mathrm{~d}, J=9.0 \mathrm{~Hz}, 1 \mathrm{H}), 7.83(\mathrm{~d}, J=9.0 \mathrm{~Hz}, 2 \mathrm{H}), 2.78(\mathrm{~s}, 3 \mathrm{H}) ;{ }^{13} \mathbf{C} \mathbf{N M R}$ $\left(\mathrm{CDCl}_{3}, 100 \mathrm{MHz}\right) \delta 153.4,149.6,124.8,124.6,44.0$.<smiles>CS(=O)c1ccc(C(=O)O)cc1</smiles>

4-(Methylsulfinyl)benzoic acid (3o ${ }^{15}$ : Known compound. Flash chromatography (200-300 mesh silica gel, eluent: EtOAc/MeOH = 3:1, v/v). 43 mg, $47 \%$ yield. White solid. m.p.: 227.0-233.0 ${ }^{\circ} \mathrm{C} .{ }^{1} \mathbf{H}$ NMR (DMSO- $d_{6}, 400$ MHz) $\delta 13.27(\mathrm{~s}, 1 \mathrm{H}), 8.10(\mathrm{~d}, J=8.04 \mathrm{~Hz}, 2 \mathrm{H}), 7.80(\mathrm{~d}, J=8.04 \mathrm{~Hz}, 2 \mathrm{H}), 2.78(\mathrm{~s}$, $3 \mathrm{H}) ;{ }^{13} \mathrm{C}$ NMR $\left(\mathrm{DMSO}-d_{6}, 100 \mathrm{MHz}\right) \delta 166.6,151.3,132.8,130.0,123.9,43.1$.<smiles>CC(=O)c1ccc(S(C)=O)cc1</smiles>

1-(4-(Methylsulfinyl)phenyl)ethan-1-one (3p) $)^{13}$ : Known compound. Flash chromatography (200-300 mesh silica gel, eluent: petroleum ether $\left(60-90{ }^{\circ} \mathrm{C}\right) / \mathrm{EtOAc}$ $=1: 4, \mathrm{v} / \mathrm{v}) .61 \mathrm{mg}, 67 \%$ yield. White solid. m.p.: 108.0-109.0 ${ }^{\circ} \mathrm{C} .{ }^{\mathbf{1}} \mathbf{H}$ NMR $\left(\mathrm{CDCl}_{3}\right.$, $400 \mathrm{MHz}) \delta 8.02(\mathrm{~d}, J=8.2 \mathrm{~Hz}, 2 \mathrm{H}), 7.67(\mathrm{~d}, J=8.2 \mathrm{~Hz}, 2 \mathrm{H}), 2.69(\mathrm{~s}, 3 \mathrm{H}), 2.57$ (s, 3H); ${ }^{13} \mathrm{C}$ NMR $\left(\mathrm{CDCl}_{3}, 100 \mathrm{MHz}\right) \delta 196.9,150.9,138.9,129.0,123.6,43.7,26.7$. 
<smiles>O=S(c1ccccc1)C1CC1</smiles>

(Cyclopropylsulfinyl)benzene $\quad(\mathbf{3 q})^{13}$ : Known compound. Flash chromatography (200-300 mesh silica gel, eluent: petroleum ether $\left(60-90{ }^{\circ} \mathrm{C}\right) / \mathrm{EtOAc}$ $=1: 1, \mathrm{v} / \mathrm{v}) .46 \mathrm{mg}, 55 \%$ yield. Colorless oil. ${ }^{\mathbf{1}} \mathbf{H} \mathbf{N M R}\left(\mathrm{CDCl}_{3}, 400 \mathrm{MHz}\right) \delta$ 7.64-7.61 (m, 2H), 7.50-7.43 (m, 2H), 2.26-2.19 (m, 1H), 1.22-1.16 (m, 1H), 1.02-0.95 (m, 1H), 0.95-0.85 (m, 2H); ${ }^{13} \mathbf{C ~ N M R ~}\left(\mathrm{CDCl}_{3}, 100 \mathrm{MHz}\right) \delta 144.8,131.0,129.2,124.0,33.8$, $3.4,2.8$.<smiles>O=S(Cc1ccccc1)c1ccccc1</smiles>

(Benzylsulfinyl)benzene (3r) ${ }^{13}$ : Known compound. Flash chromatography (200-300 mesh silica gel, eluent: petroleum ether $\left(60-90{ }^{\circ} \mathrm{C}\right) /$ EtOAc $\left.=1: 1, \mathrm{v} / \mathrm{v}\right) .52$ mg, 48\% yield. White solid. m.p.: 122.0-123.0 ${ }^{\circ} \mathrm{C} .{ }^{1} \mathbf{H} \mathbf{N M R}\left(\mathrm{CDCl}_{3}, 400\right.$ MHz) $\delta$ 7.48-7.36 (m, 5H), 7.30-7.22 (m, 3H), $6.98(\mathrm{~d}, J=7.0 \mathrm{~Hz}, 2 \mathrm{H}), 4.09$ (d, $J=$ $12.6 \mathrm{~Hz}, 1 \mathrm{H}), 3.99(\mathrm{~d}, J=12.6 \mathrm{~Hz}, 1 \mathrm{H}) ;{ }^{13} \mathbf{C} \mathbf{N M R}\left(\mathrm{CDCl}_{3}, 100 \mathrm{MHz}\right)$ $\delta 142.8,131.3,130.4,129.2,128.9,128.5,128.3,124.5,63.7$.<smiles>CS(=O)c1ccccn1</smiles>

2-(Methylsulfinyl)pyridine (3s) ${ }^{13}$ : Known compound. Flash chromatography (200-300 mesh silica gel, eluent: petroleum ether $\left.\left(60-90{ }^{\circ} \mathrm{C}\right) / \mathrm{EtOAc}=1: 2, \mathrm{v} / \mathrm{v}\right) .9 \mathrm{mg}$, 13\% yield. Yellow oil. ${ }^{1} \mathbf{H}$ NMR $\left(\mathrm{CDCl}_{3}, 400 \mathrm{MHz}\right) \delta 8.53(\mathrm{~s}, 1 \mathrm{H}), 7.93-7.83(\mathrm{~m}, 2 \mathrm{H})$,

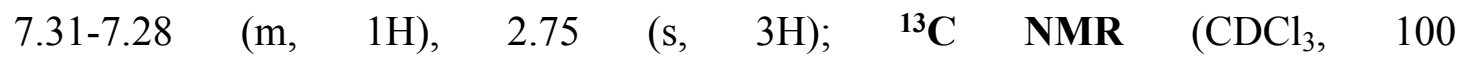
$\mathrm{MHz}) \delta 165.7,149.4,138.1,124.6,119.1,41.2$.<smiles>CS(=O)c1nc(Cl)cc(Cl)n1</smiles>

4,6-Dichloro-2-(methylsulfinyl)pyrimidine (3t) ${ }^{16}$ : Known compound. Flash 
chromatography (200-300 mesh silica gel, eluent: petroleum ether $\left(60-90{ }^{\circ} \mathrm{C}\right) / \mathrm{EtOAc}$ $=1: 2, \mathrm{v} / \mathrm{v}) .17 \mathrm{mg}, 16 \%$ yield. White solid. m.p.: 81.0-83.0 ${ }^{\circ} \mathrm{C} .{ }^{1} \mathbf{H} \mathbf{~ N M R}\left(\mathrm{CDCl}_{3}, 400\right.$ MHz) $\delta 7.47(\mathrm{~s}, 1 \mathrm{H}), 2.97(\mathrm{~s}, 3 \mathrm{H}) ;{ }^{13} \mathbf{C} \mathbf{N M R}\left(\mathrm{CDCl}_{3}, 100 \mathrm{MHz}\right) \delta$ 175.7, 163.6, $122.2,40.5$<smiles>CS(=O)(=O)c1ccc(C(=O)OC2COCCO2)cc1</smiles>

1,4-Dioxan-2-yl 4-(methylsulfonyl)benzoate (4) ${ }^{2 a}$ : Known compound. Flash chromatography (200-300 mesh silica gel, eluent: EtOAc $/ \mathrm{MeOH}=50: 1, \mathrm{v} / \mathrm{v}) .100$ mg, 70\% yield. Colorless oil. ${ }^{1} \mathbf{H}$ NMR $\left(\mathrm{CDCl}_{3}, 400 \mathrm{MHz}\right) \delta 8.26-8.24(\mathrm{~m}, 2 \mathrm{H})$, 7.73-7.71 (m, 2H), $6.09(\mathrm{~s}, 1 \mathrm{H}), 4.22-4.16(\mathrm{~m}, 1 \mathrm{H}), 3.88(\mathrm{~d}, J=1.8 \mathrm{~Hz}, 2 \mathrm{H})$, 3.83-3.80 (m, 2H), 3.69-3.64 (m, 1H), $2.74(\mathrm{~s}, 3 \mathrm{H})$.<smiles>C(=C/c1ccccc1)\c1ccccc1</smiles>

(E)-1,2-Diphenylethene $(5)^{2 b}$ : Known compound. Flash chromatography (200-300 mesh silica gel, eluent: petroleum ether $\left.\left(60-90{ }^{\circ} \mathrm{C}\right) / \mathrm{EtOAc}=10: 1, \mathrm{v} / \mathrm{v}\right) .73$ mg, 81\% yield. White solid. m.p.: 124.7-126.1 ${ }^{\circ} \mathrm{C} .{ }^{1} \mathbf{H}$ NMR $\left(\mathrm{CDCl}_{3}, 400\right.$ MHz) $\delta$ 7.57-7.54 (m, 4H), 7.42-7.38 (m, 4H), 7.32-7.28 (m, 2H), $7.16(\mathrm{~s}, 2 \mathrm{H})$.<smiles>O=C(Cc1ccccc1Sc1ccccc1)c1ccccc1</smiles>

1-Phenyl-2-(2-(phenylthio)phenyl)ethan-1-one (6) ${ }^{2 c}$ : Known compound. Flash chromatography (200-300 mesh silica gel, eluent: petroleum ether $\left(60-90{ }^{\circ} \mathrm{C}\right) / \mathrm{EtOAc}$ $=25: 1, \mathrm{v} / \mathrm{v}) .74 \mathrm{mg}, 82 \%$ yield. Yellow oil. ${ }^{1} \mathbf{H}$ NMR $\left(\mathrm{CDCl}_{3}, 400 \mathrm{MHz}\right) \delta$ 7.96-7.94 (m, 2H), 7.54-7.50 (m, 1H), 7.45-7.38 (m, 3H), 7.30-7.19 (m, 5H), 7.15-7.11 (m, 3H), $4.47(\mathrm{~s}, 2 \mathrm{H})$. 
<smiles>COC(=O)c1ccccc1</smiles>

Triphenylsulfonium trifluoromethanesulfonate $\left(\mathbf{7}^{2 \mathrm{~d}}\right.$ : Known compound. Flash chromatography (200-300 mesh silica gel, eluent: DCM/MeOH = 5:1, v/v). 134 mg, $65 \%$ yield. White solid. m. p.: 130.0-131.0 ${ }^{\circ} \mathrm{C} .{ }^{1} \mathbf{H}$ NMR $\left(\mathrm{CDCl}_{3}, 400\right.$ $\mathrm{MHz}) \delta$ 7.91-7.72 (m, 15H).

\section{References}

(1) Li, Y. M.; Nie, C. P.; Wang, H. F.; Li, X. Y.; Verpoort, F.; Duan, C. Y. Eur. J. Org. Chem. 2011, 2011, 7331-7338.

(2) (a) Chen, L.; Shi, E.; Liu, Z.; Chen, S.; Wei, W.; Li, H.; Xu, K.; Wan, X. Chem. Eur. J. 2011, 17, 4085-4089. (b) Yao, C.-Z.; Li, Q.-Q.; Wang, M.-M.; Ning, X.-S.; Kang, Y.-B. Chem. Commun. 2015, 51, 7729-7732. (c) Kaiser, D.; Veiros, L. F.; Maulide, N. Chem. Eur. J. 2016, 22, 4727-4732. (d) Ma, W.; Li, S.; Zhou, Z.; Shen, H.; Li, X.; Sun, Q.; He, L.; Xue, Y. Eur. J. Org. Chem. 2012, 2012, $1554-1562$.

(3) Yang, Y. Q.; Chen, Z.; Rao, Y. Chem. Commun. 2014, 50, 15037-15040.

(4) Feng, J.-B.; Gong, J.-L.; Wu, X.-F. RSC Adv. 2014, 4, 29273-29275.

(5) Jereb, M. Green Chem. 2012, 14, 3047-3052.

(6) Yuan, G. Q.; Zheng, J. H.; Gao, X. F.; Li, X. W.; Huang, L. B.; Chen, H. J.; Jiang, H. F. Chem. Commun., 2012, 48, 7513-7515.

(7) Kar, A.; Sayyed, I. A.; Lo, W. F.; Kaiser, H. M.; Beller, M.; Tse, M. K. Org. Lett. 2007, 9, 3405-3408.

(8) Mandal, M.; Chakraborty, D. RSC Adv. 2015, 5, 12111-12122.

(9) Joseph, J. T.; Sajith, A. M.; Ningegowda, R. C.; Shashikanth, S. Adv. Synth. Catal. 2017, 359, 419-425.

(10) Robke, L.; Laraia, L.; Corrales, M. A. C.; Konstantinidis, G.; Muroi, M.; Richters, A.; Winzker, M.; Engbring, T.; Tomassi, S.; Watanabe, N.; Osada, H.; Rauh, D.; Waldmann, H.; Wu, Y.-W.; Engel, J. Angew. Chem. Int. Ed. 2017, 56, 8153-8157. 
(11) Yu, H.; Li, Z.; Bolm, C. Org. Lett. 2018, 20, 7104-7106.

(12) Chun, J.-H.; Morse, C. L.; Chin, F. T.; Pike, V. W. Chem. Commun. 2013, 49, 2151-2153.

(13) Gan, S. Y.; Yin, J. J.; Yao, Y.; Liu, Y.; Chang, D. H.; Zhu, D.; Shi, L. Org. Biomol. Chem. 2017, 15, 2647-2654.

(14) Dai, W.; Li, G. S.; Wang, L.Y.; Chen, B.; Shang, S. S.; Lv, Y.; Gao, S. RSC Adv. 2014, 4, 46545-46554.

(15) Hu, Y.-L.; Fang, D.; Xing, R. RSC Adv. 2014, 4, 51140-51145.

(16) Dai, W.; Shang, S. S.; Lv, Y.; Li, G. S.; Li, C. S. ACS Catal. 2017, 7, 4890-4895. 
6. Copies of NMR spectra

${ }^{1} \mathrm{H}$ NMR of product $2 \mathrm{a}$ in $\mathrm{CDCl}_{3}(400 \mathrm{MHz})$
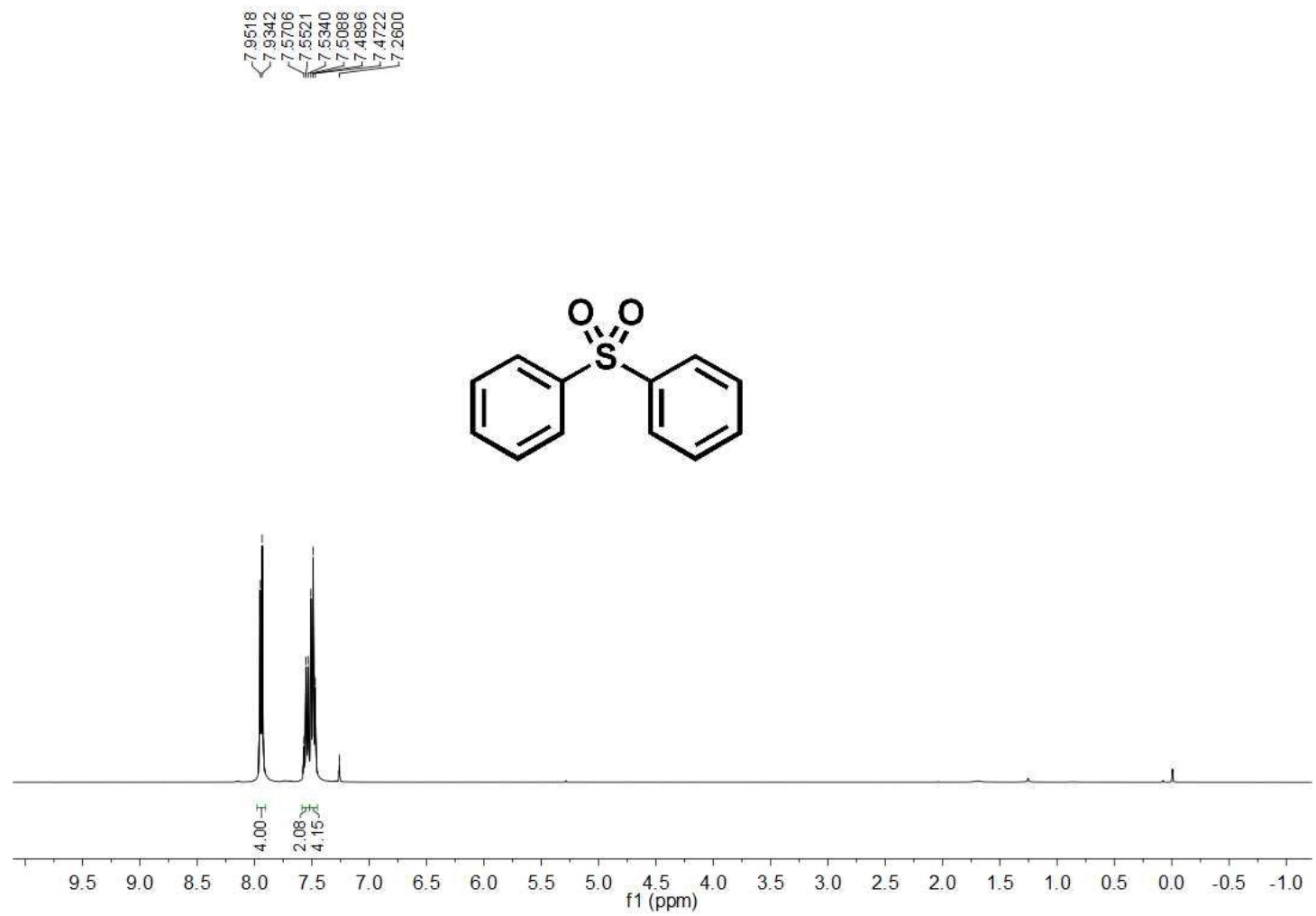

${ }^{13} \mathrm{C}$ NMR of product $2 \mathrm{a}$ in $\mathrm{CDCl}_{3}(100 \mathrm{MHz})$ ए गुलू

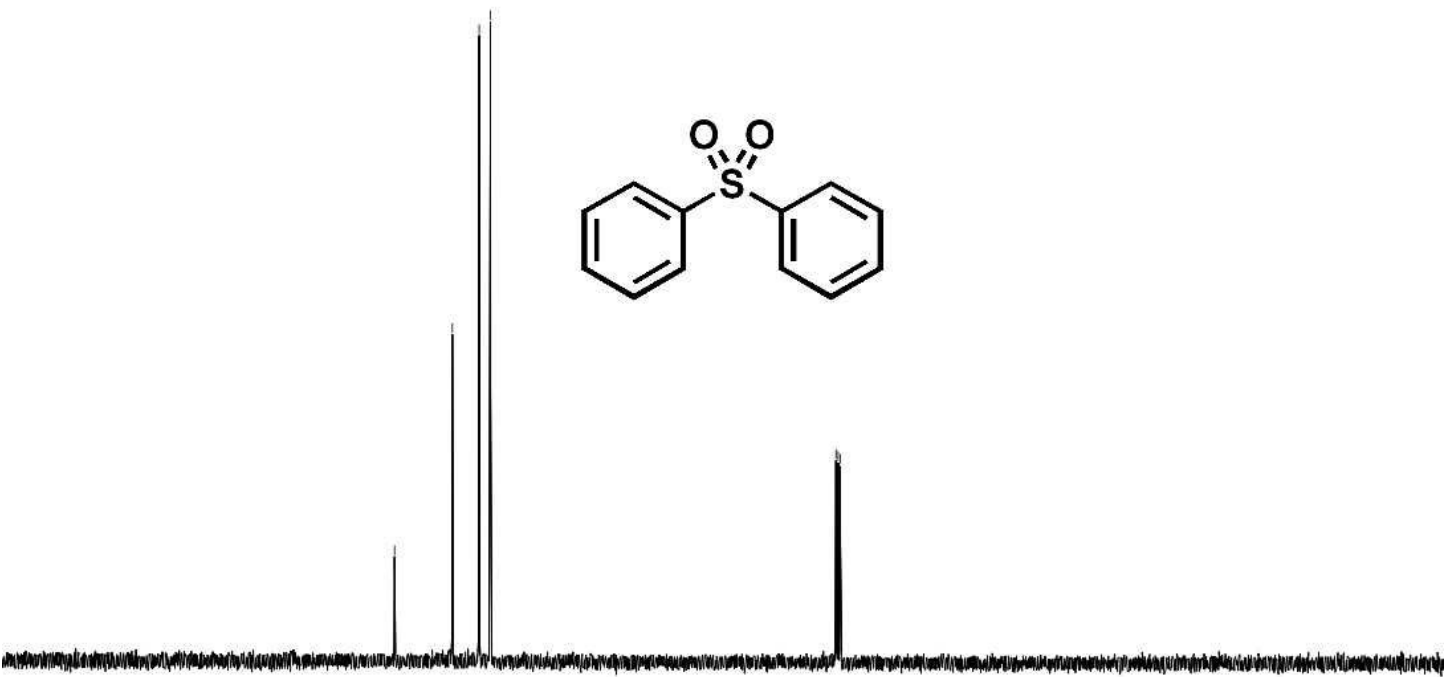

$\begin{array}{llllllllll}190 & 180 & 170 & 160 & 150 & 140 & 130 & 120 & 110 & \begin{array}{r}100 \\ \mathrm{f} 1(\mathrm{ppm})\end{array}\end{array}$ 
${ }^{1} \mathrm{H}$ NMR of product $2 \mathrm{~b}$ in $\mathrm{CDCl}_{3}(400 \mathrm{MHz})$
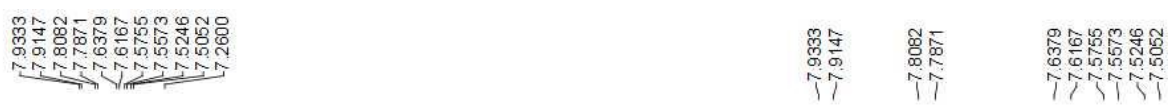<smiles>O=S(=O)(c1ccccc1)c1ccc(Br)cc1</smiles>
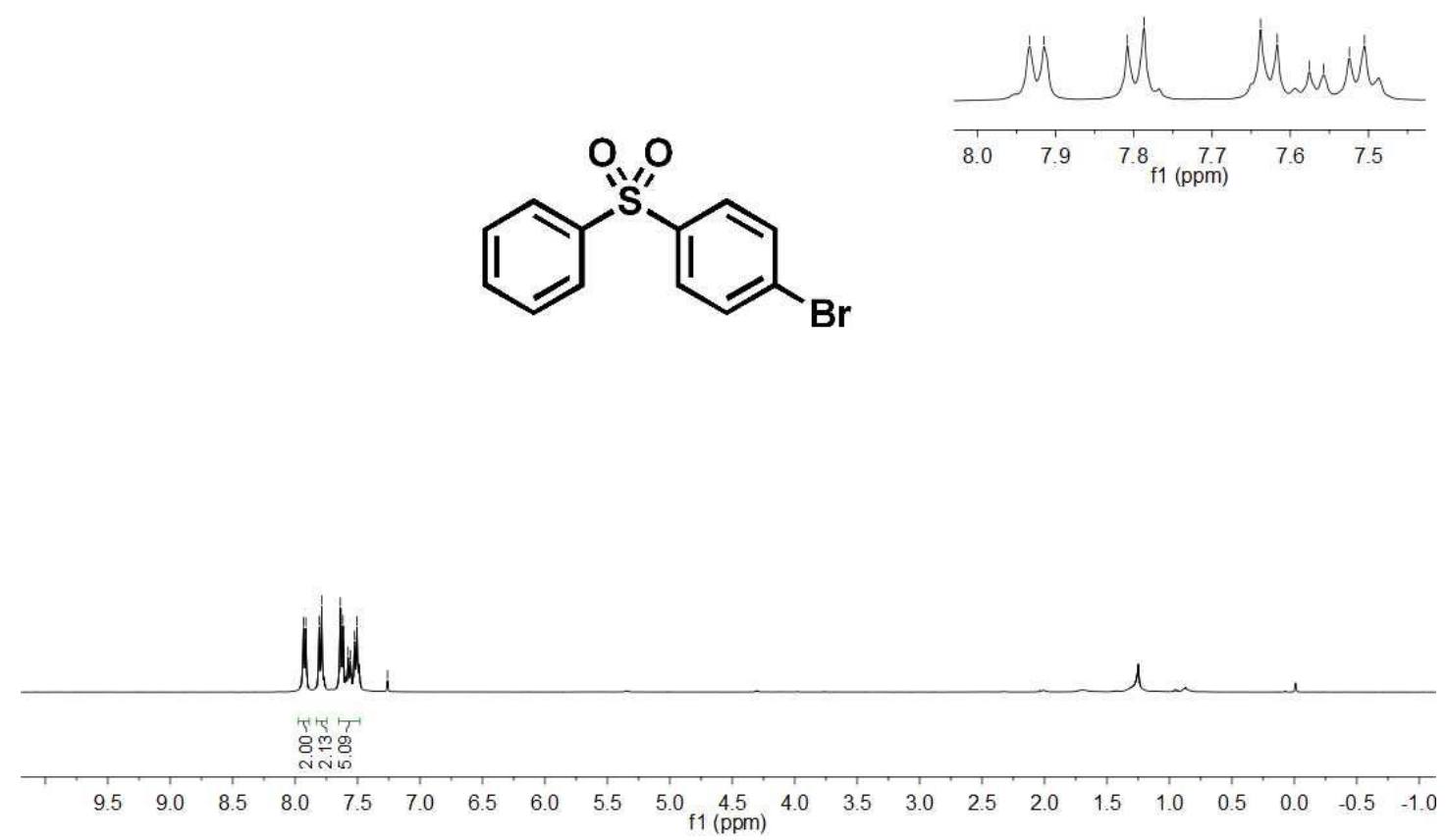

${ }^{13} \mathrm{C}$ NMR of product $2 \mathrm{~b}$ in $\mathrm{CDCl}_{3}(100 \mathrm{MHz})$

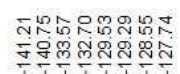

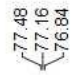<smiles>O=S(=O)(c1ccccc1)c1ccc(Br)cc1</smiles>

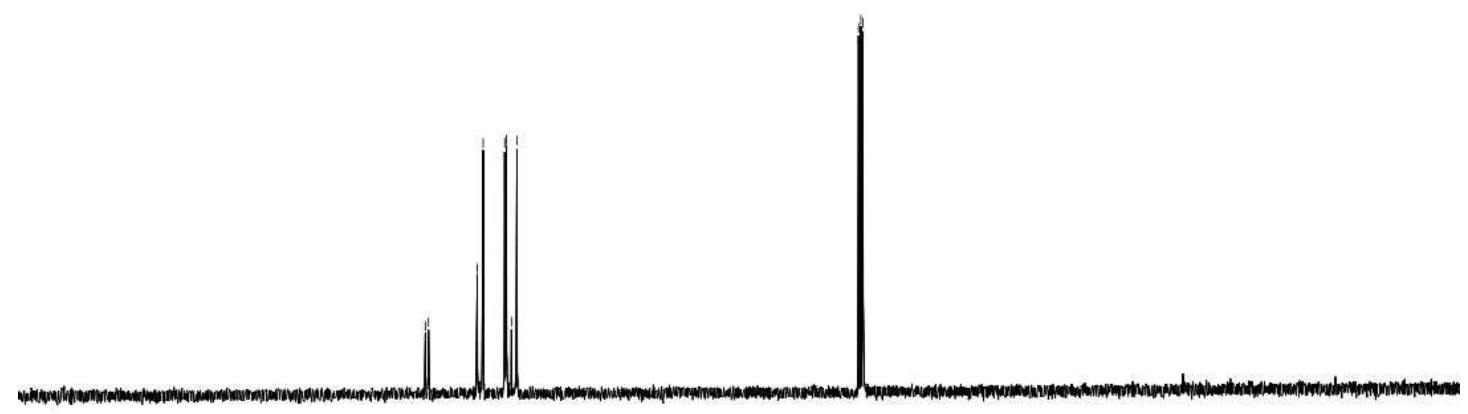

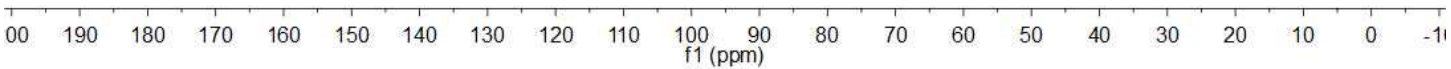


${ }^{1} \mathrm{H}$ NMR of product $2 \mathrm{c}$ in $\mathrm{CDCl}_{3}(400 \mathrm{MHz})$
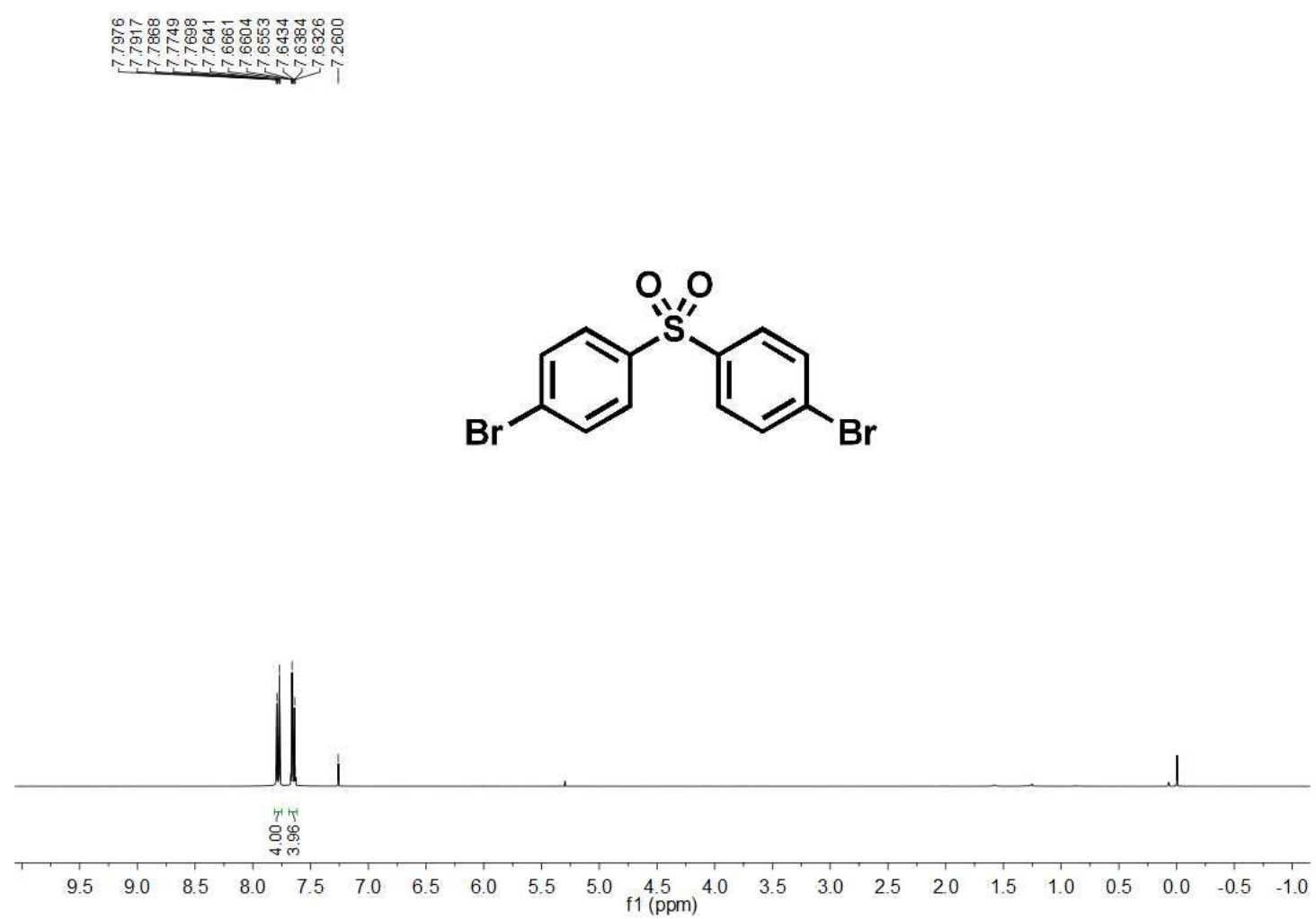

${ }^{13} \mathrm{C}$ NMR of product $2 \mathrm{c}$ in $\mathrm{CDCl}_{3}(100 \mathrm{MHz})$

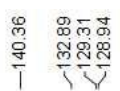

邹

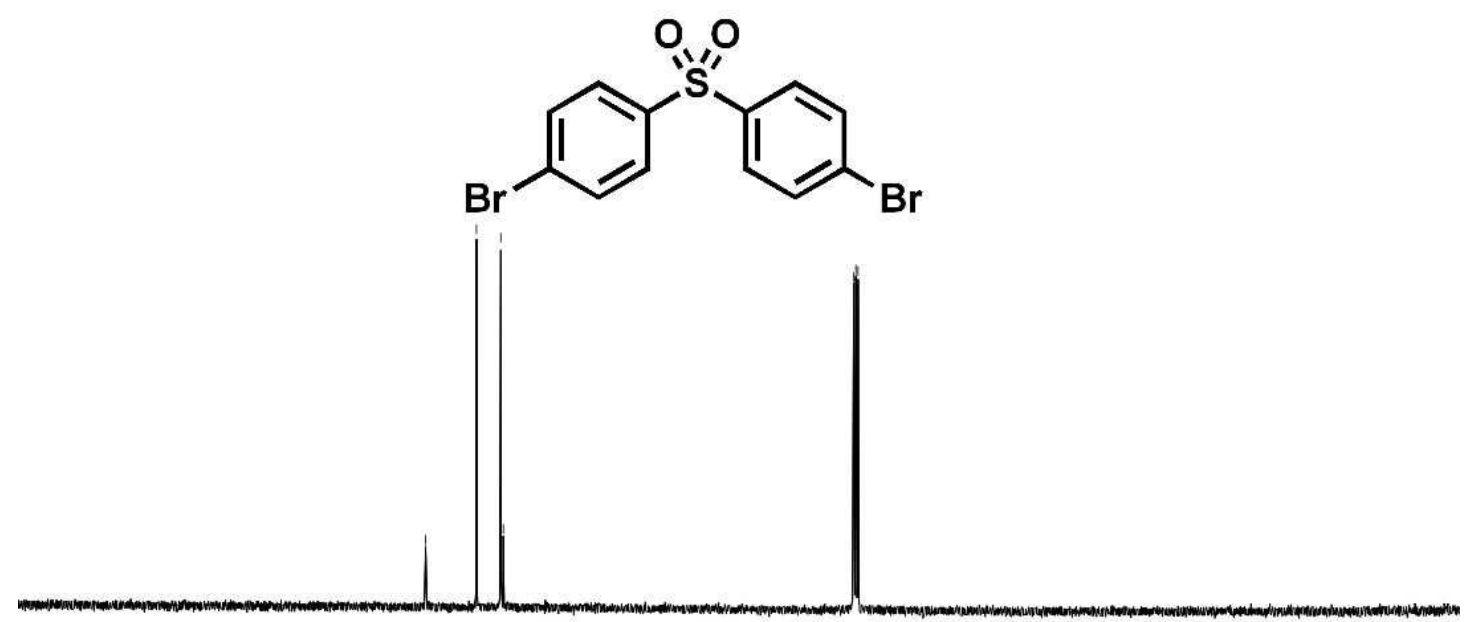

\begin{tabular}{lllllllllllllllllllllllll}
\hline 0 & 190 & 180 & 170 & 160 & 150 & 140 & 130 & 120 & 110 & $\begin{array}{r}100 \\
\mathrm{f} 1(\mathrm{ppm})\end{array}$ & 80 & 70 & 60 & 50 & 40 & 30 & 20 & 10 & 0 & -16
\end{tabular} 
${ }^{1} \mathrm{H}$ NMR of product 2d in $\mathrm{CDCl}_{3}(400 \mathrm{MHz})$
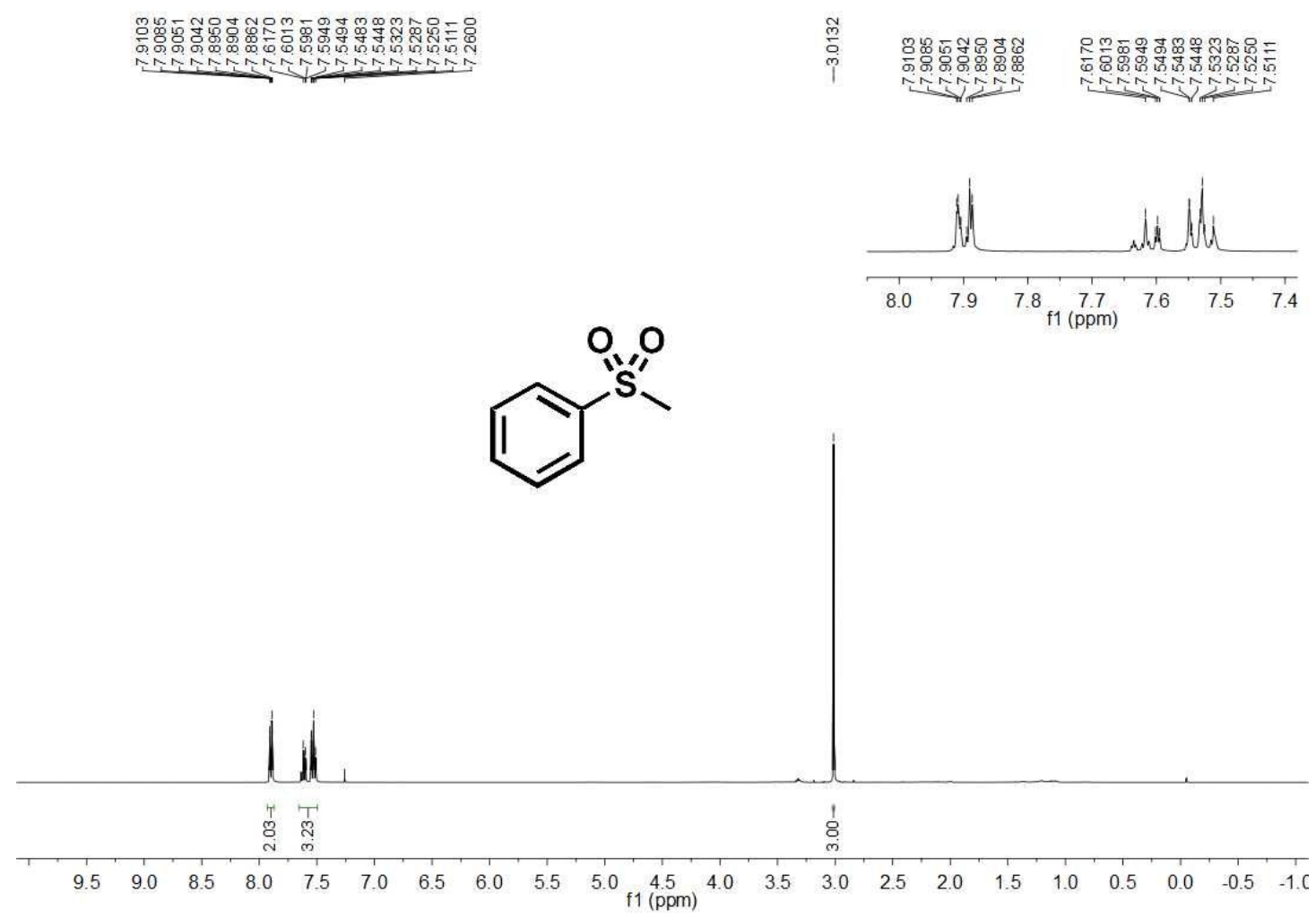

${ }^{13} \mathrm{C}$ NMR of product $2 \mathrm{~d}$ in $\mathrm{CDCl}_{3}(100 \mathrm{MHz})$

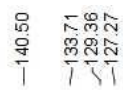

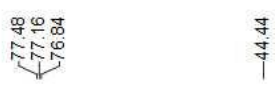

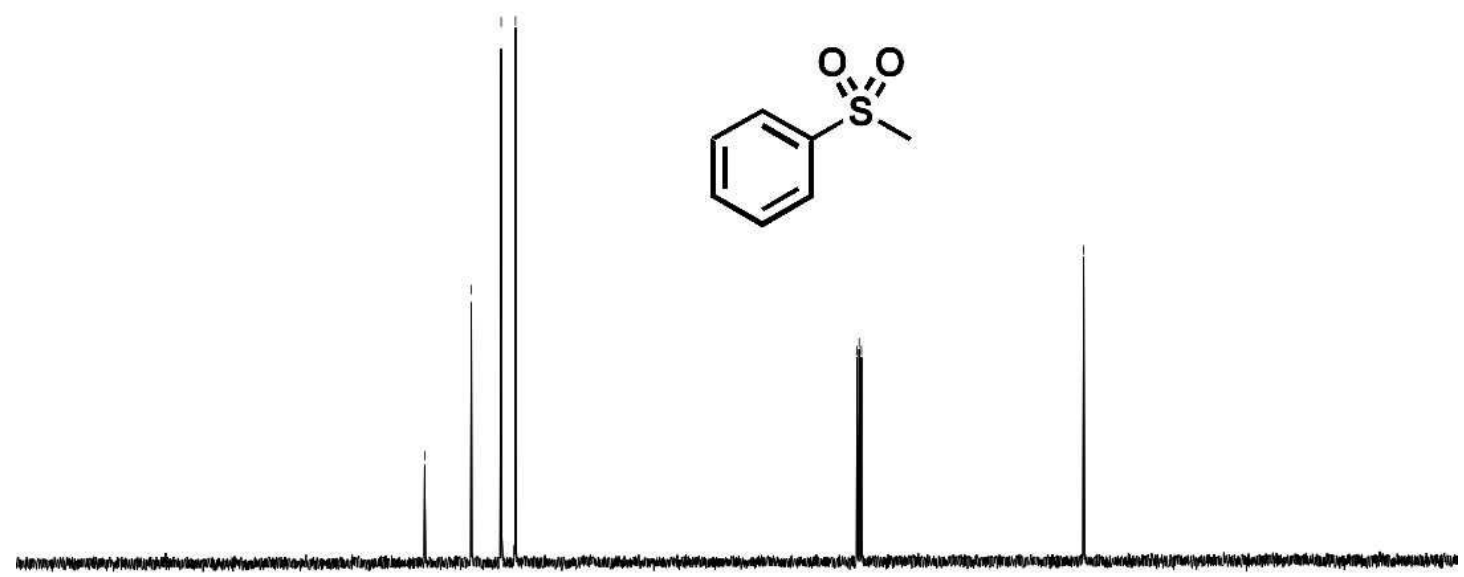

$\begin{array}{lllllllllll}30 & 190 & 180 & 170 & 160 & 150 & 140 & 130 & 120 & 110 & 100 \\ \mathrm{f} 1(\mathrm{ppm}) & 90\end{array}$ 
${ }^{1} \mathrm{H}$ NMR of product 2e in $\mathrm{CDCl}_{3}(400 \mathrm{MHz})$

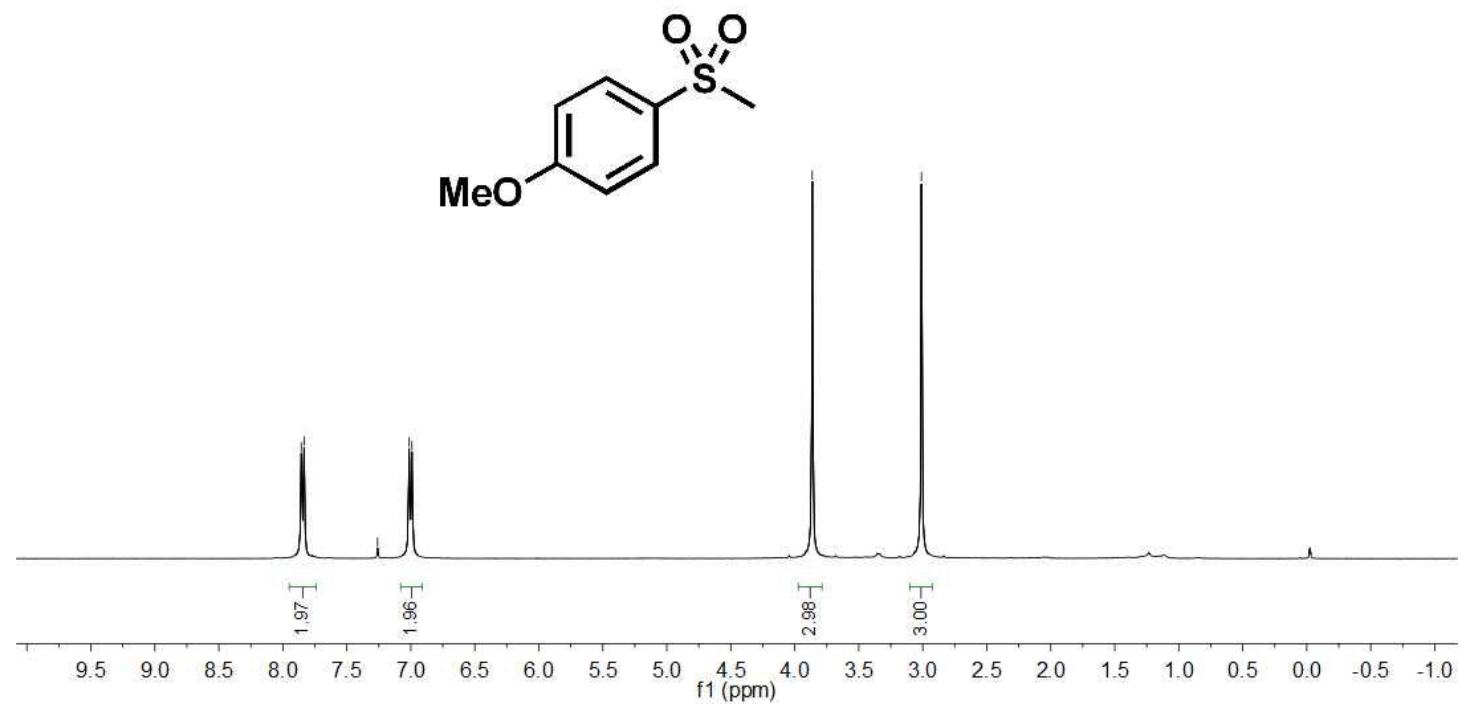

${ }^{13} \mathrm{C}$ NMR of product $2 \mathrm{e}$ in $\mathrm{CDCl}_{3}(100 \mathrm{MHz})$

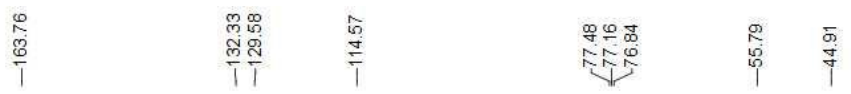

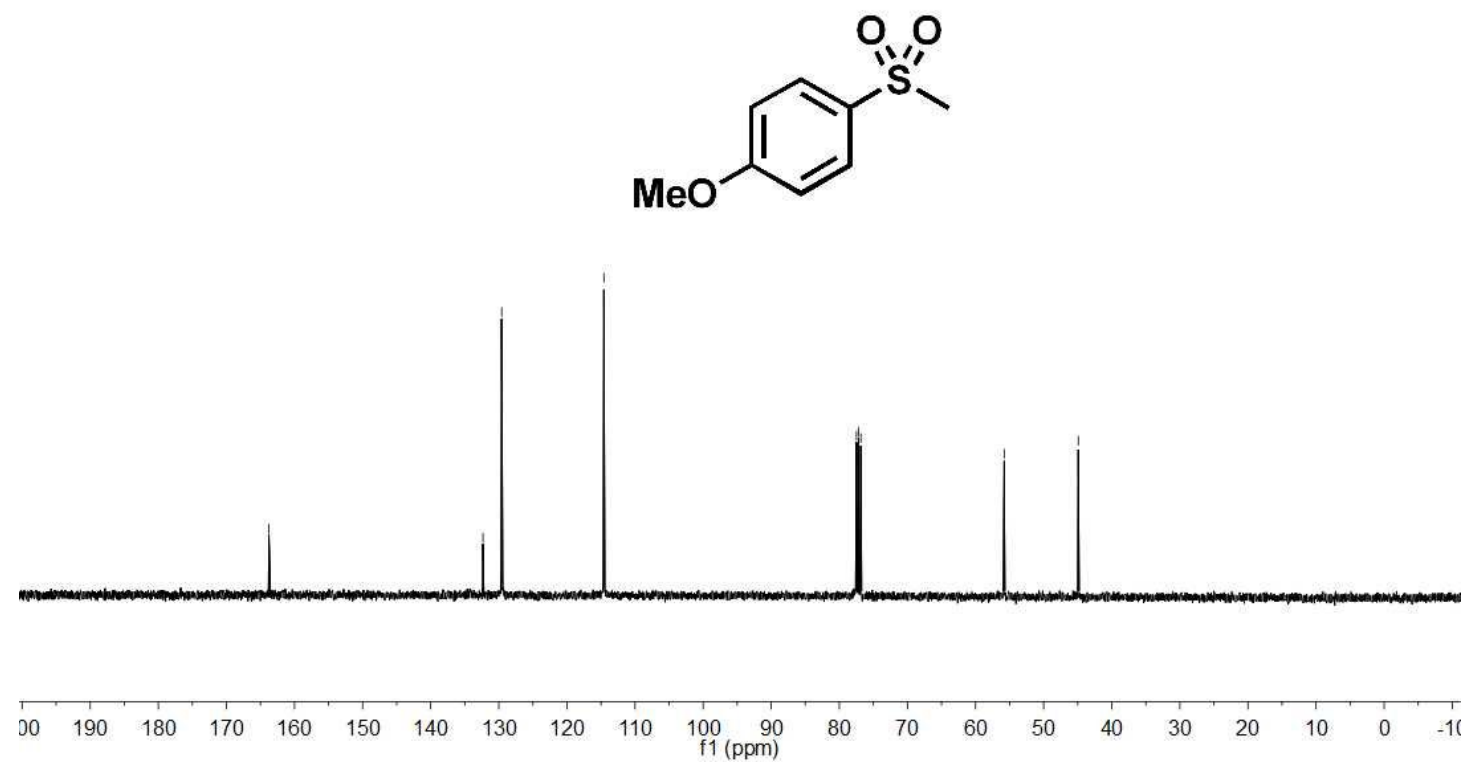


${ }^{1} \mathrm{H}$ NMR of product $2 \mathrm{f}$ in $\mathrm{CDCl}_{3}(400 \mathrm{MHz})$

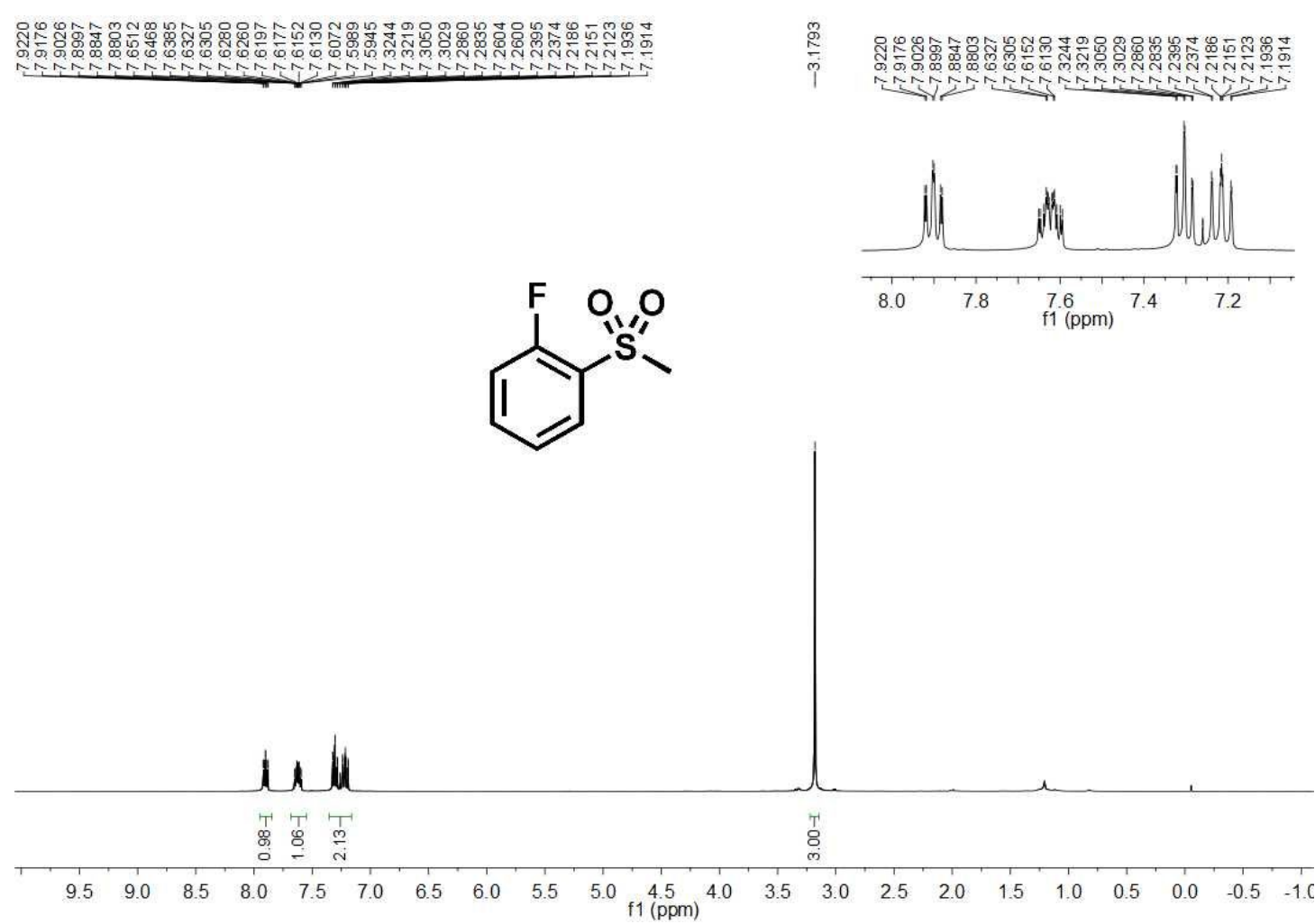

${ }^{13} \mathrm{C}$ NMR of product $2 \mathrm{f}$ in $\mathrm{CDCl}_{3}(100 \mathrm{MHz})$

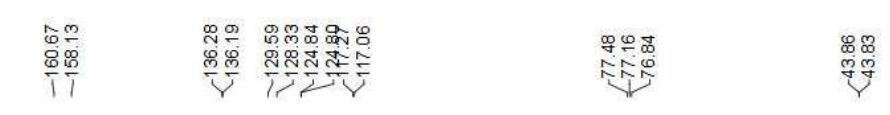

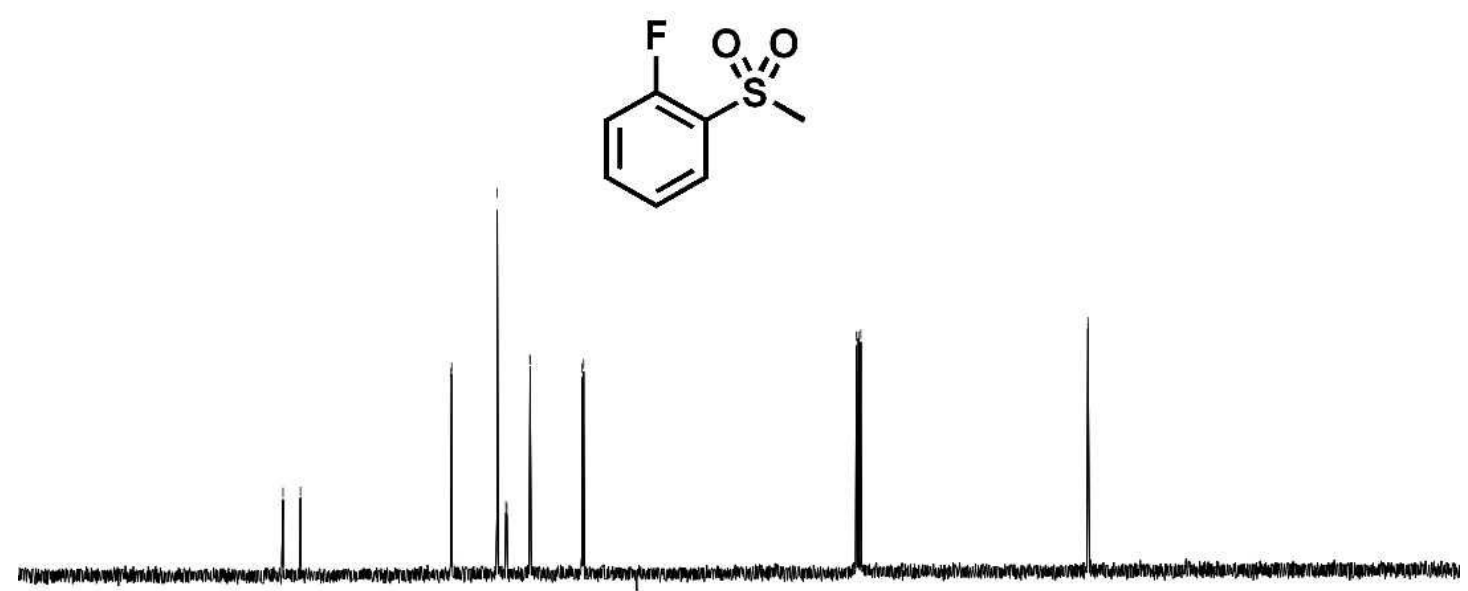

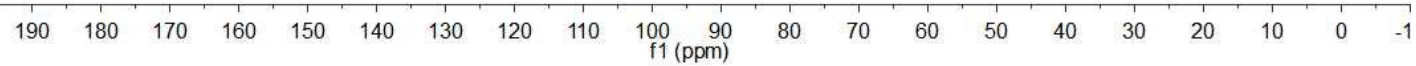


${ }^{1} \mathrm{H}$ NMR of product $2 \mathrm{~g}$ in $\mathrm{CDCl}_{3}(400 \mathrm{MHz})$

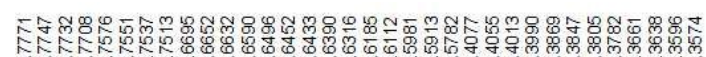

ANAR

$\stackrel{8}{\stackrel{8}{\circ}}$

is

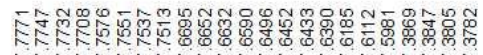

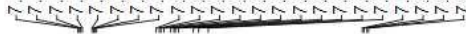

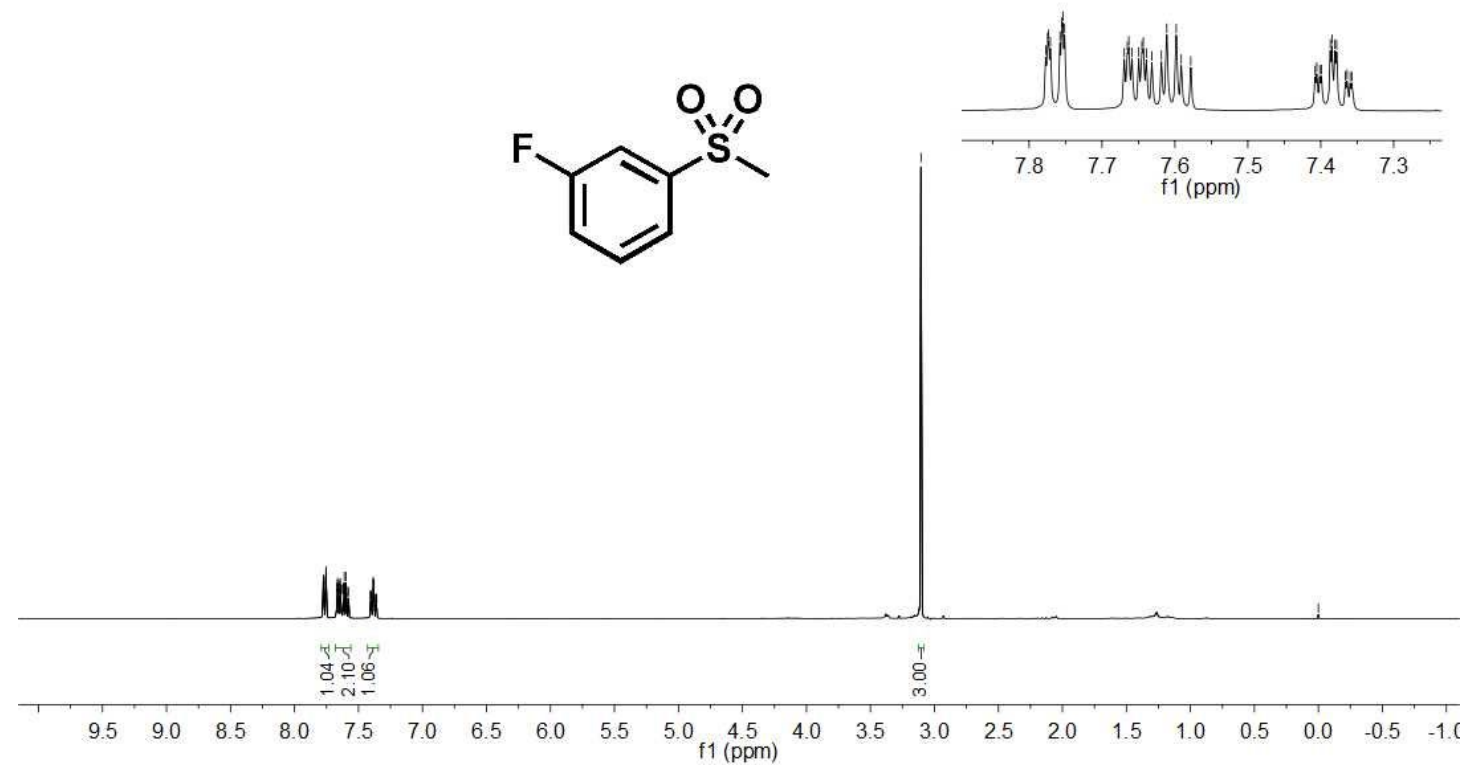

${ }^{13} \mathrm{C}$ NMR of product $2 \mathrm{~g}$ in $\mathrm{CDCl}_{3}(100 \mathrm{MHz})$

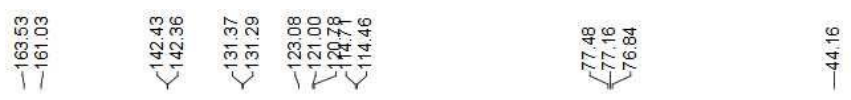

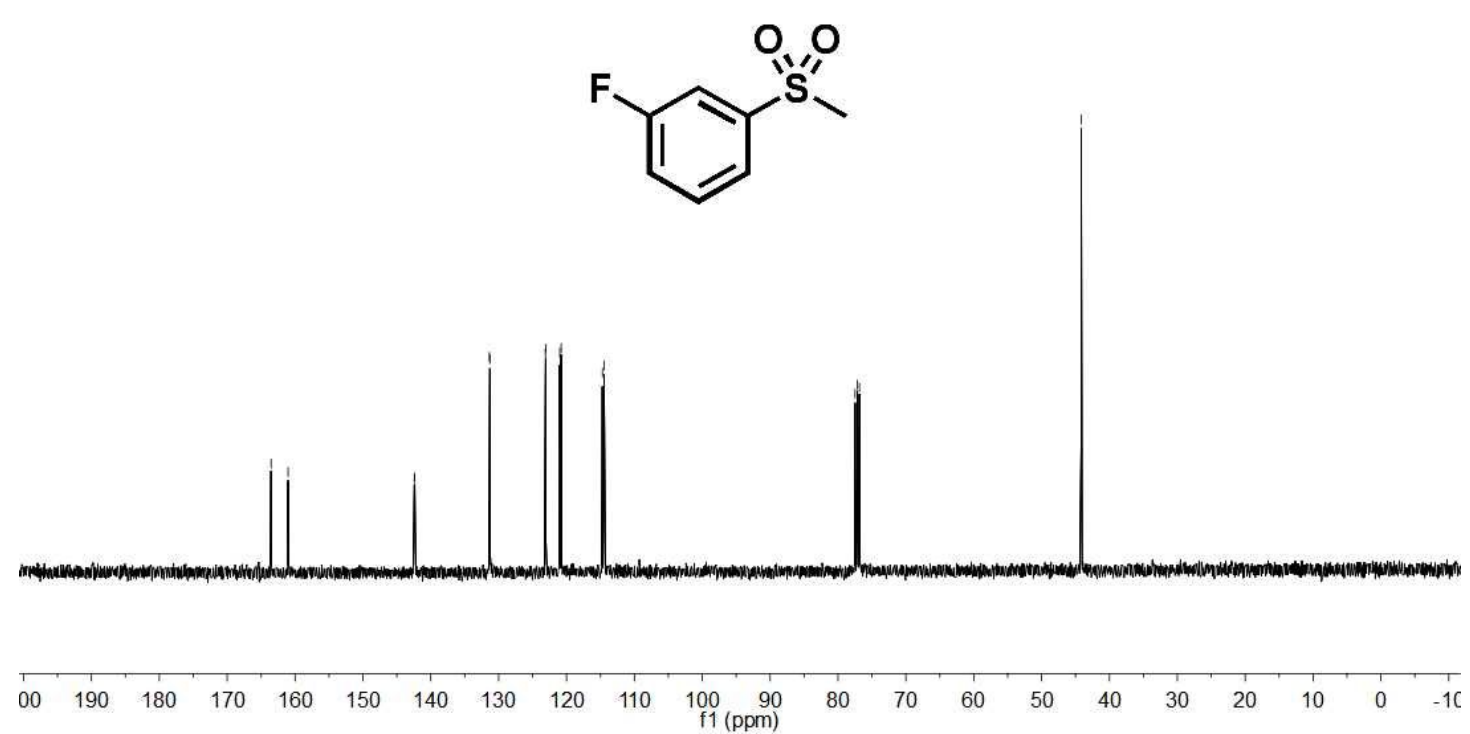


${ }^{1} \mathrm{H}$ NMR of product $2 \mathrm{~h}$ in $\mathrm{CDCl}_{3}(400 \mathrm{MHz})$

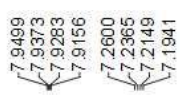<smiles>CS(=O)(=O)c1ccc(F)cc1</smiles>

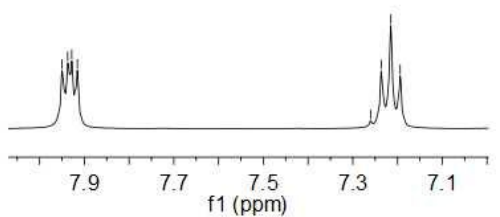

$\mathrm{F}$

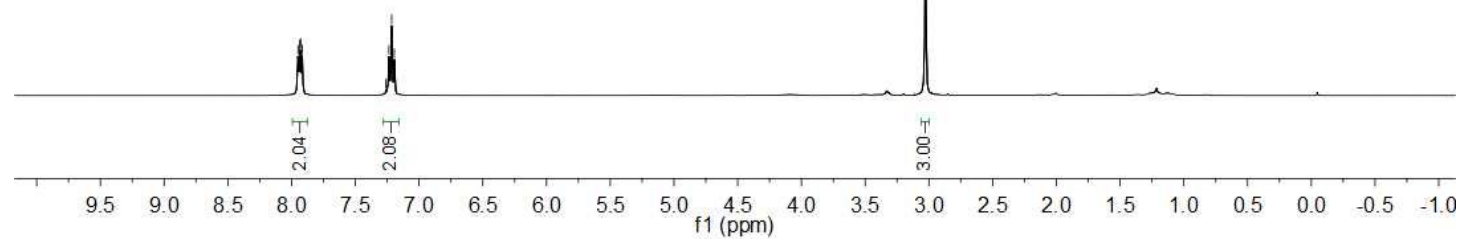

${ }^{13} \mathrm{C}$ NMR of product $2 \mathrm{~h}$ in $\mathrm{CDCl}_{3}(100 \mathrm{MHz})$

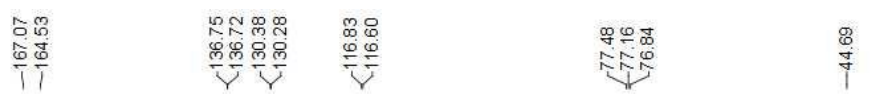<smiles>CS(=O)(=O)c1ccc(F)cc1</smiles>

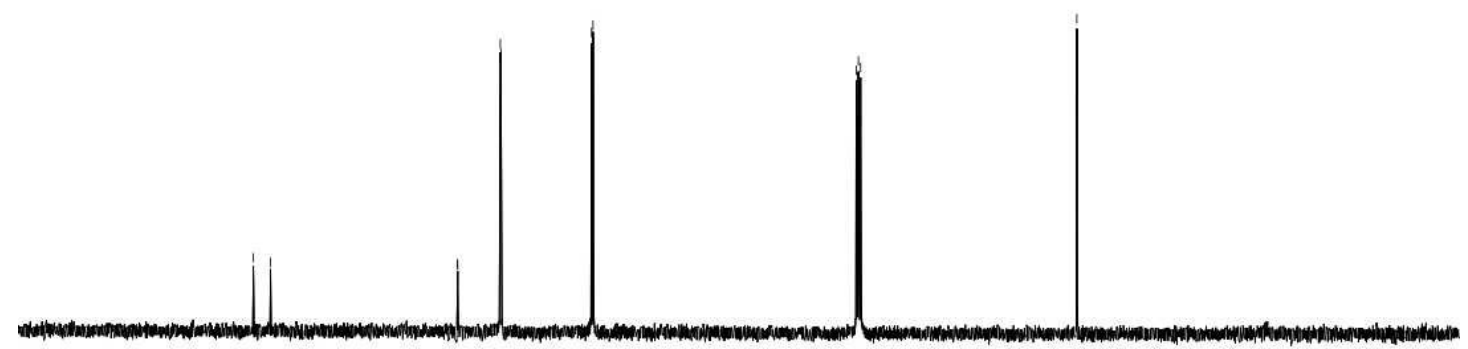

$\begin{array}{lllllllllll}200 & 190 & 180 & 170 & 160 & 150 & 140 & 130 & 120 & 110 & \begin{array}{c}100 \\ \mathrm{f} 1(\mathrm{ppm})\end{array}\end{array}$ 


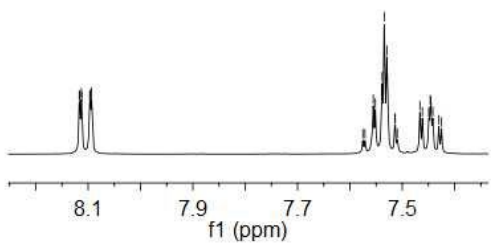<smiles>CS(=O)(=O)c1ccccc1Cl</smiles>
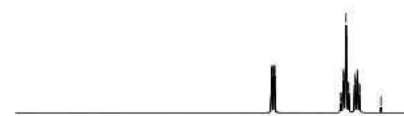

\section{T'}

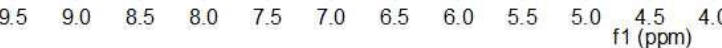

${ }^{13} \mathrm{C}$ NMR of product $2 \mathrm{i}$ in $\mathrm{CDCl}_{3}(100 \mathrm{MHz})$

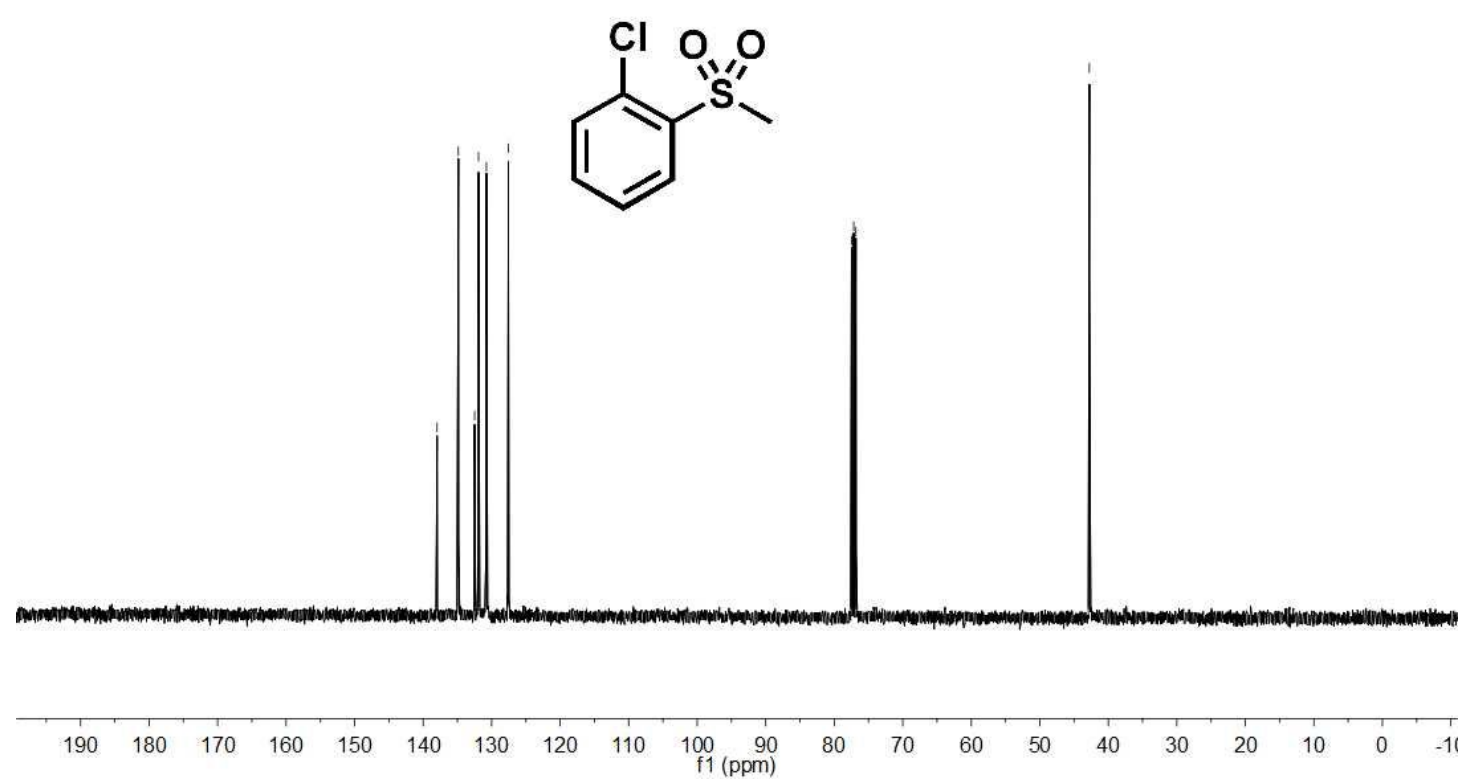


${ }^{1} \mathrm{H}$ NMR of product $2 \mathrm{j}$ in $\mathrm{CDCl}_{3}(400 \mathrm{MHz})$

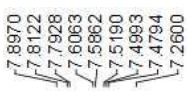<smiles>CS(=O)(=O)c1cccc(Cl)c1</smiles>
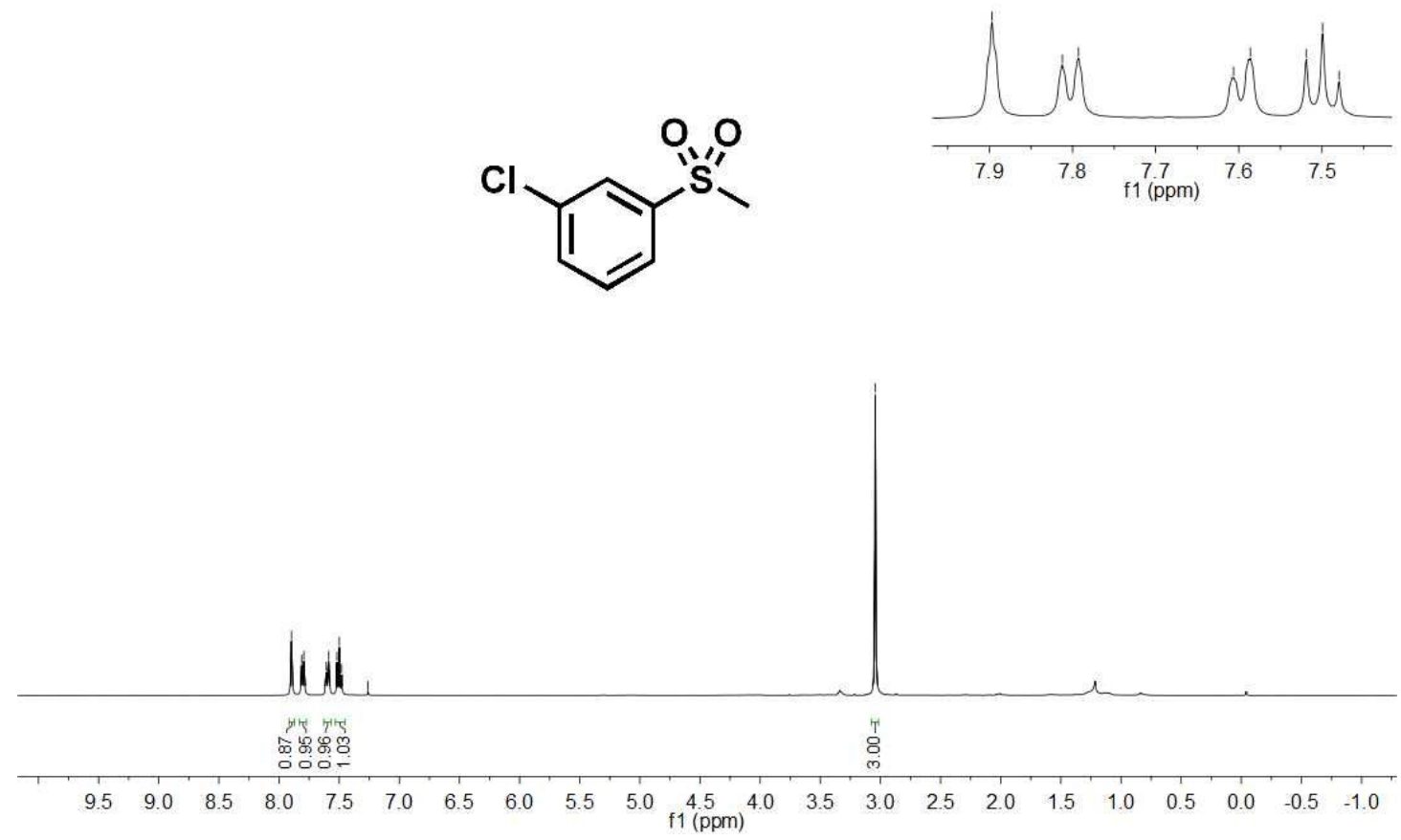

${ }^{13} \mathrm{C}$ NMR of product $2 \mathrm{j}$ in $\mathrm{CDCl}_{3}(100 \mathrm{MHz})$
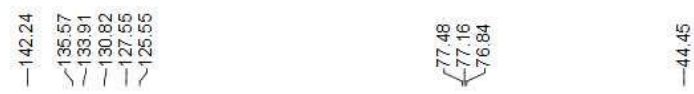<smiles>CS(=O)(=O)c1cccc(Cl)c1</smiles>

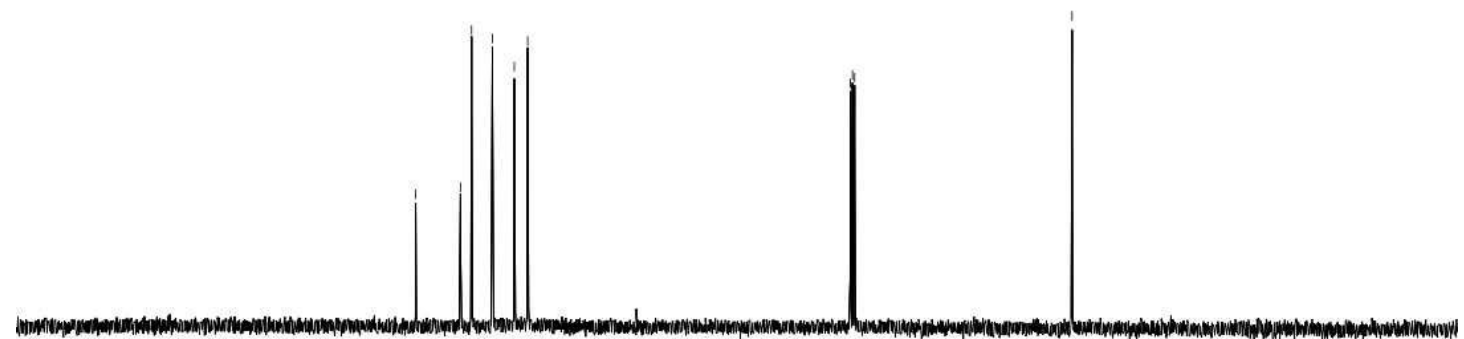

$\begin{array}{lllllllllll}200 & 190 & 180 & 170 & 160 & 150 & 140 & 130 & 120 & 110 & \begin{array}{c}100 \\ f 1(\mathrm{ppm})\end{array}\end{array}$

$\begin{array}{lllllllll}70 & 60 & 50 & 40 & 30 & 20 & 10 & 0 & -10\end{array}$ 
${ }^{1} \mathrm{H}$ NMR of product $2 \mathrm{k}$ in $\mathrm{CDCl}_{3}(400 \mathrm{MHz})$

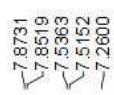

产<smiles>CS(=O)(=O)c1ccc(Cl)cc1</smiles>

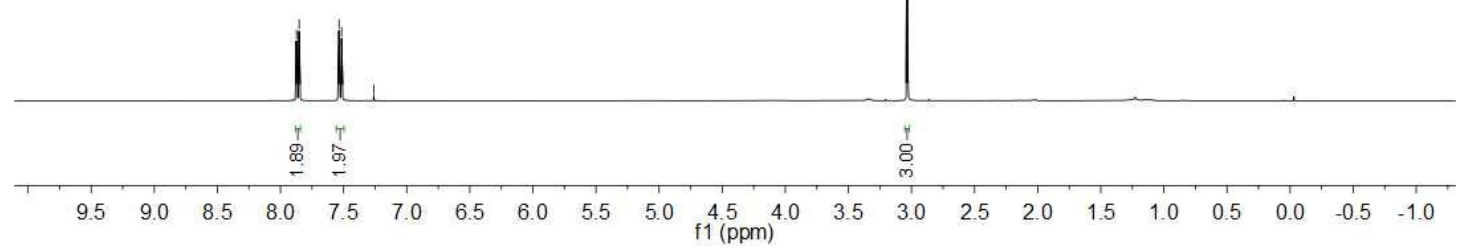

${ }^{13} \mathrm{C}$ NMR of product $2 \mathrm{k}$ in $\mathrm{CDCl}_{3}(100 \mathrm{MHz})$
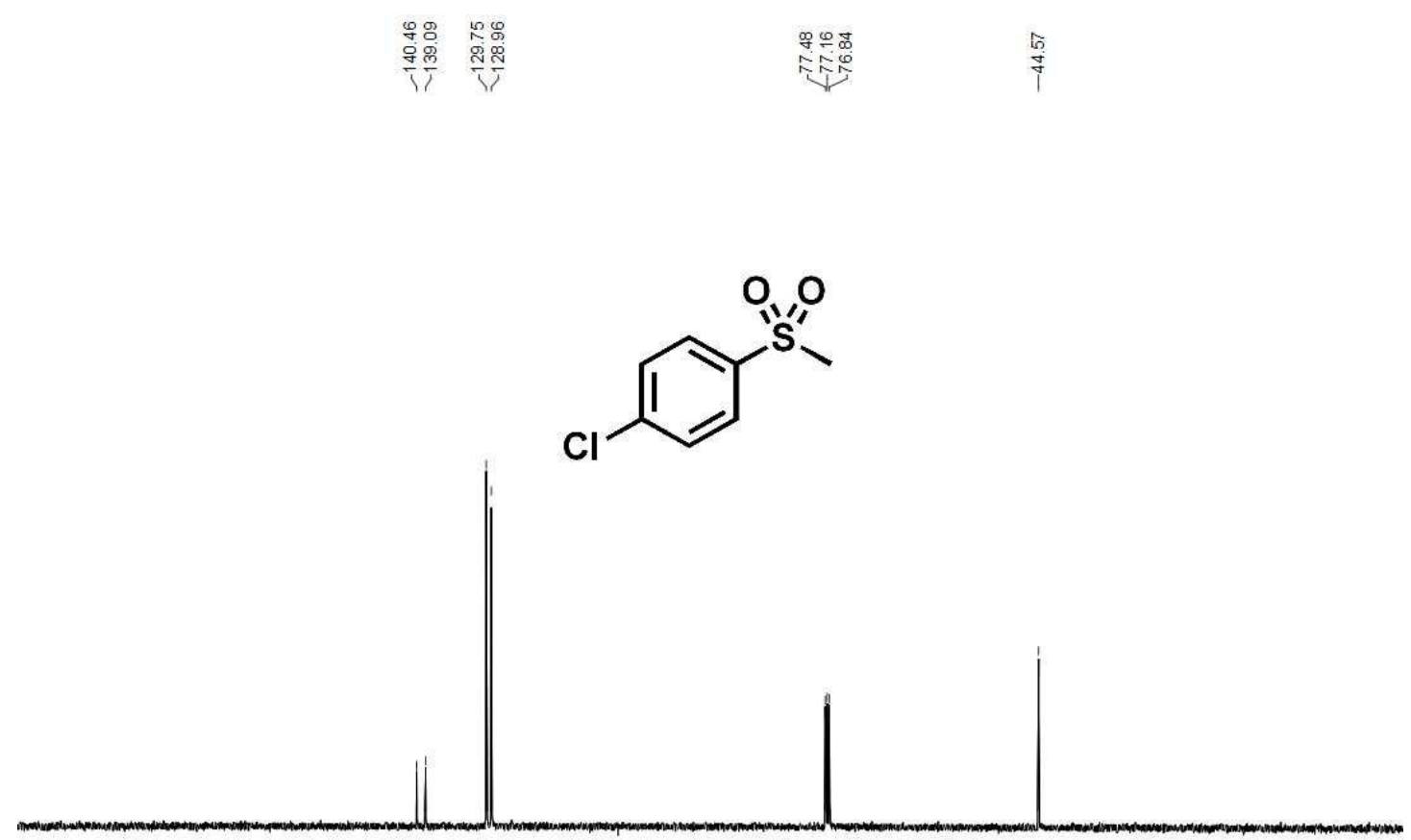

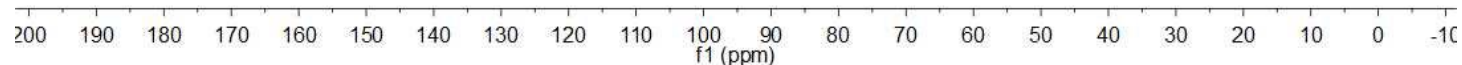


${ }^{1} \mathrm{H}$ NMR of product $2 \mathrm{l}$ in $\mathrm{CDCl}_{3}(400 \mathrm{MHz})$

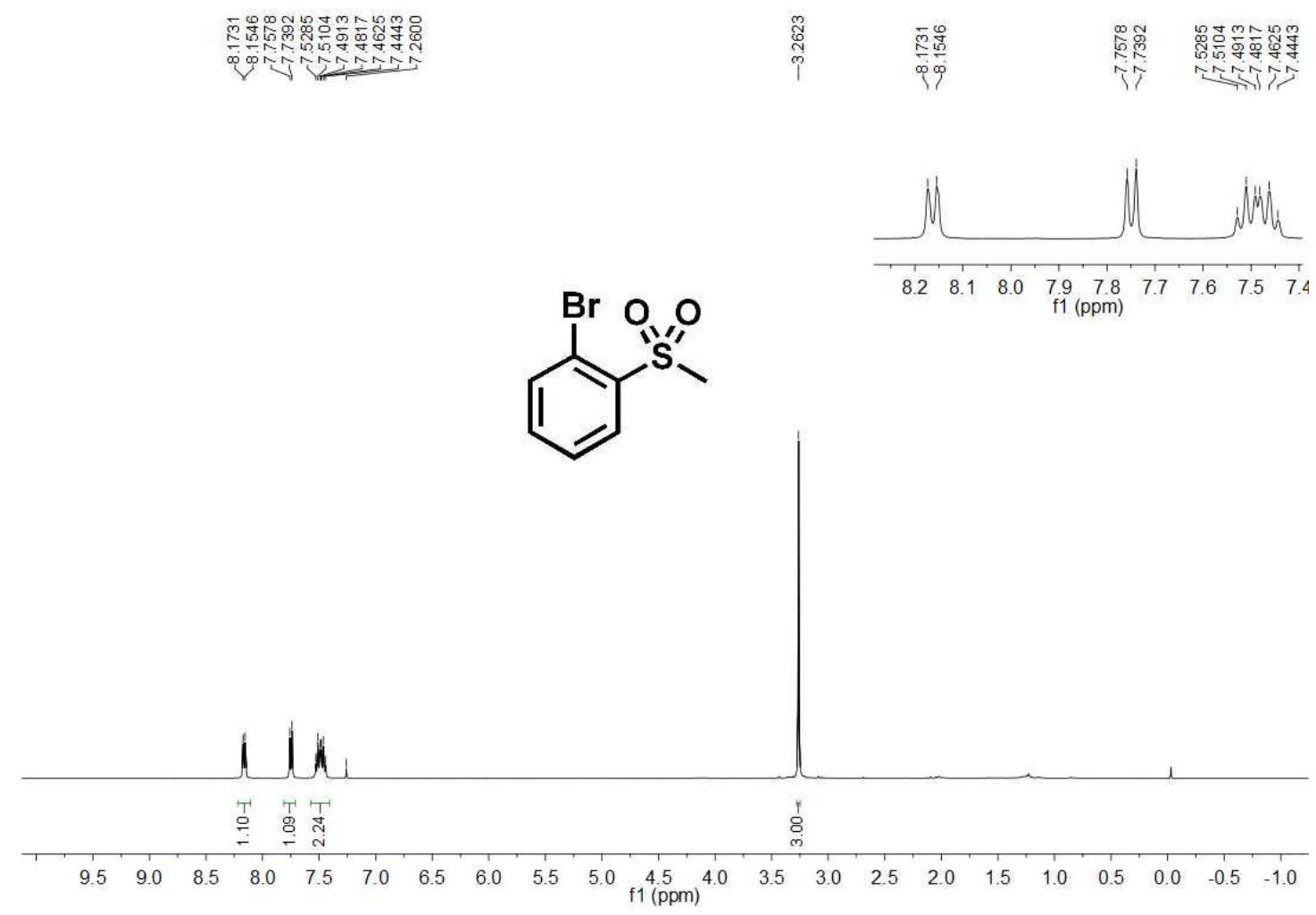

${ }^{13} \mathrm{C}$ NMR of product $2 \mathrm{I}$ in $\mathrm{CDCl}_{3}(100 \mathrm{MHz})$
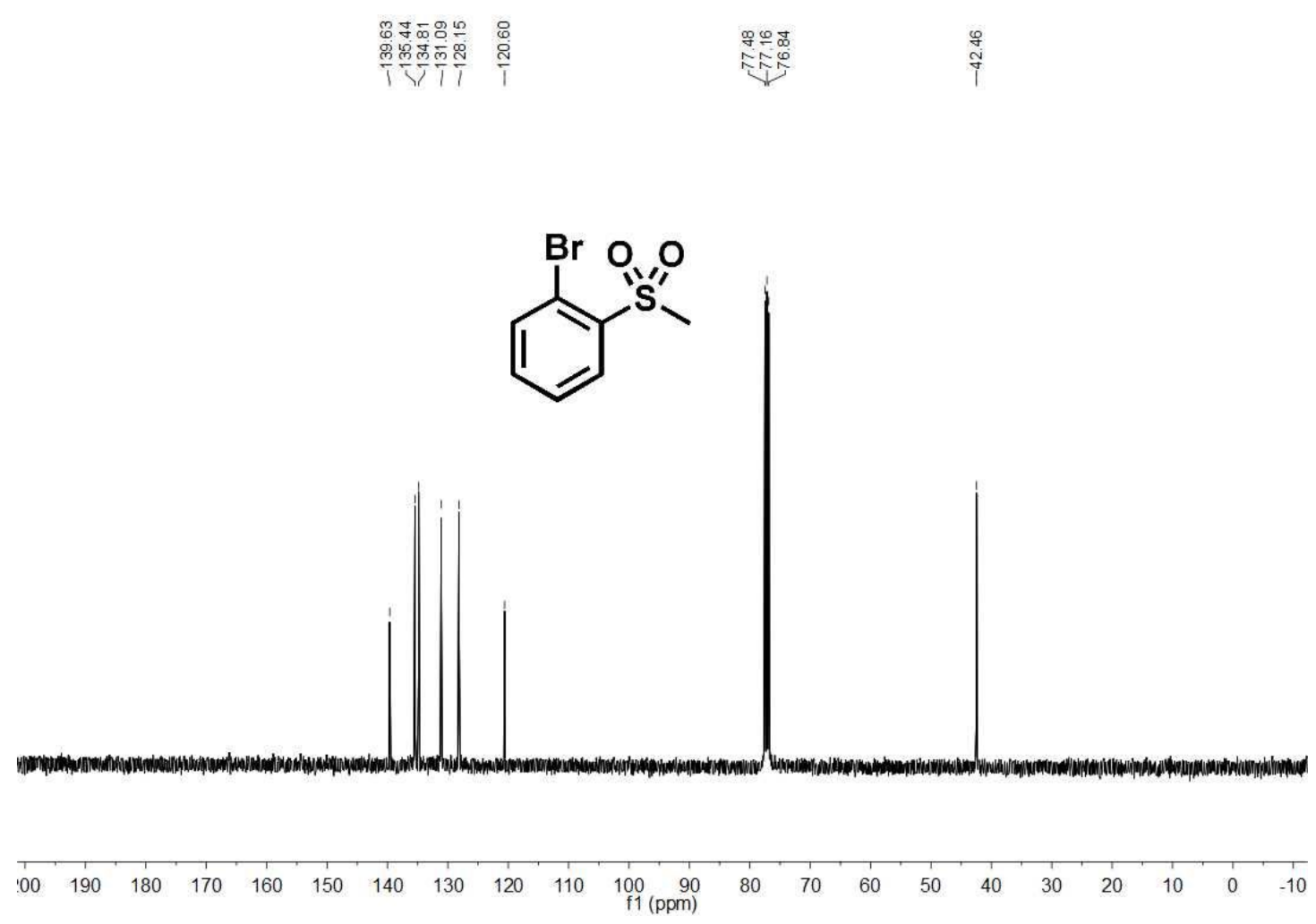
${ }^{1} \mathrm{H}$ NMR of product $2 \mathrm{~m}$ in $\mathrm{CDCl}_{3}(400 \mathrm{MHz})$

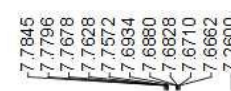<smiles>CS(=O)(=O)c1ccc(Br)cc1</smiles>
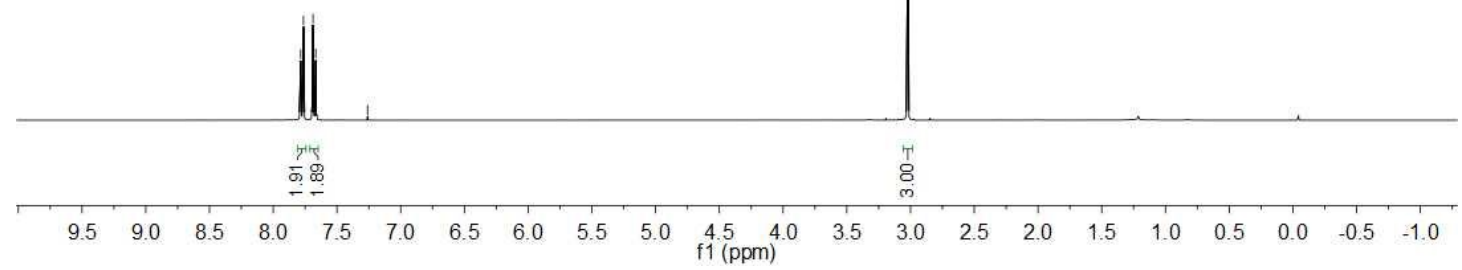

${ }^{13} \mathrm{C}$ NMR of product $2 \mathrm{~m}$ in $\mathrm{CDCl}_{3}(100 \mathrm{MHz})$

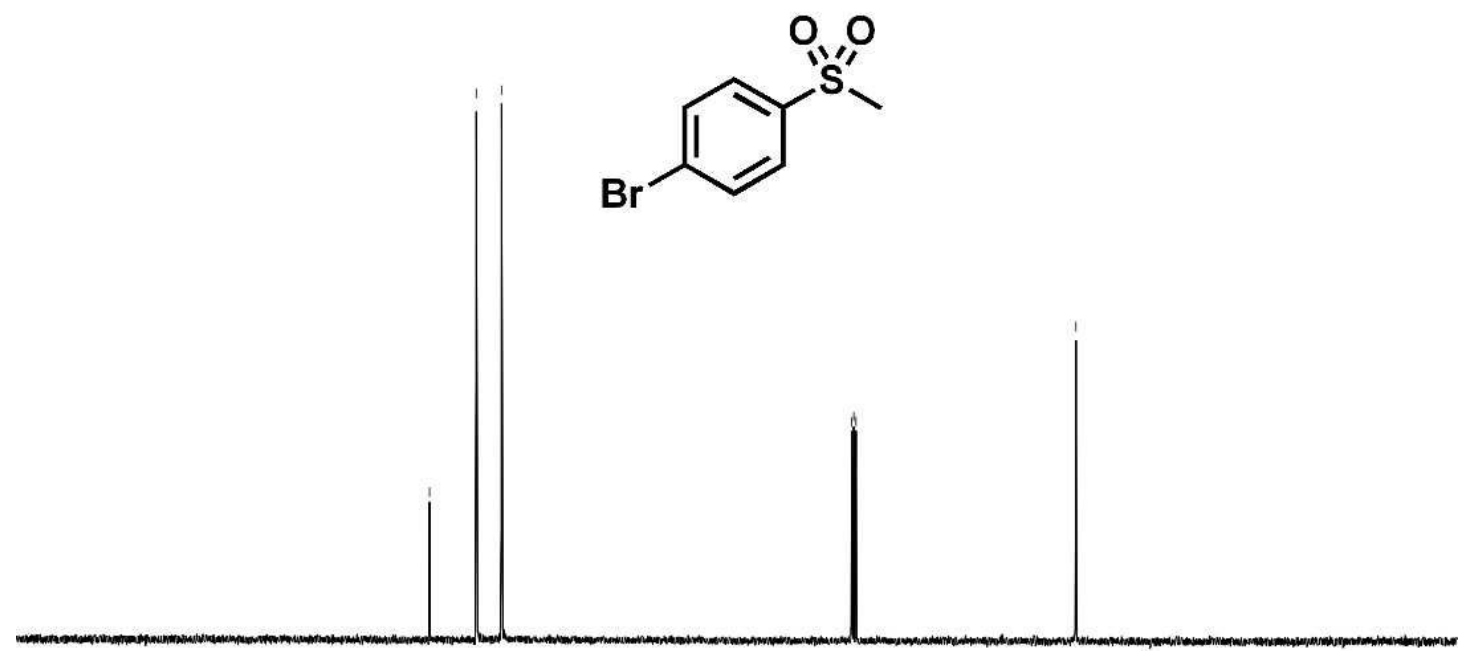

\begin{tabular}{lrrrrrrrrrrr}
\hline 00 & 190 & 180 & 170 & 160 & 150 & 140 & 130 & 120 & 110 & $\begin{array}{c}100 \\
\mathrm{f} 1(\mathrm{ppm})\end{array}$ \\
\hline
\end{tabular}

$\begin{array}{llllllllll}80 & 70 & 60 & 50 & 40 & 30 & 20 & 10 & 0 & -1 C\end{array}$ 
${ }^{1} \mathrm{H}$ NMR of product $2 \mathrm{n}$ in DMSO- $d_{6}(400 \mathrm{MHz})$

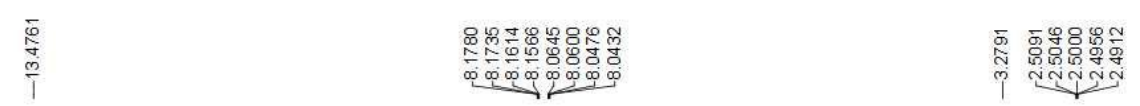<smiles>CS(=O)(=O)c1ccc(C(=O)O)cc1</smiles>

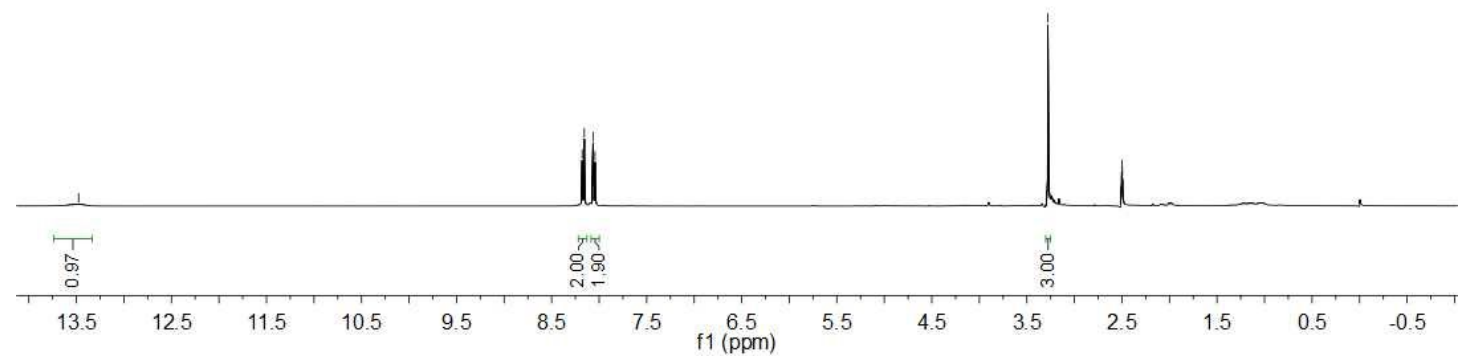

${ }^{13} \mathrm{C}$ NMR of product $2 \mathrm{n}$ in DMSO- $d_{6}(100 \mathrm{MHz})$

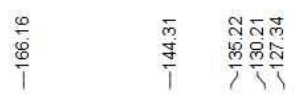<smiles>CS(=O)(=O)c1ccc(C(=O)O)cc1</smiles>

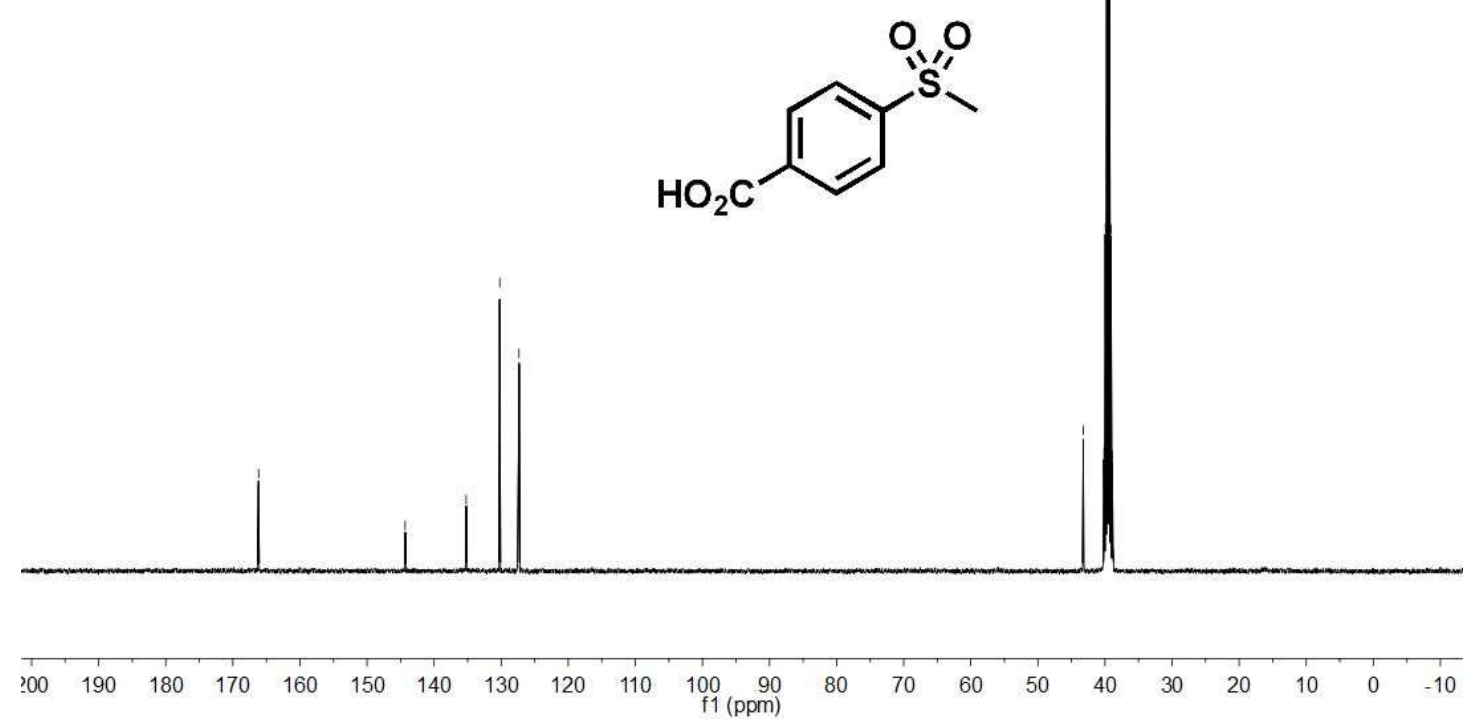


${ }^{1} \mathrm{H}$ NMR of product 20 in $\mathrm{CDCl}_{3}(400 \mathrm{MHz})$

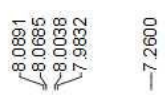

产<smiles>CC(=O)c1ccc(S(C)(=O)=O)cc1</smiles>

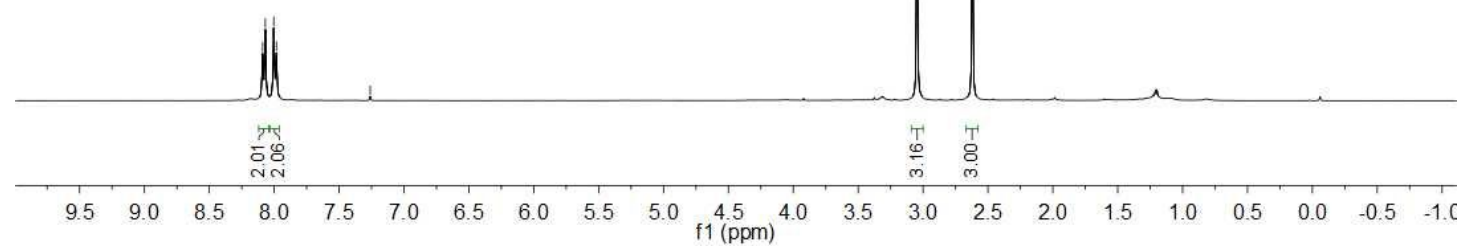

${ }^{13} \mathrm{C}$ NMR of product 20 in $\mathrm{CDCl}_{3}(100 \mathrm{MHz})$

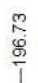

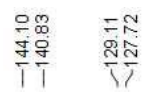

$\sqrt{4}$

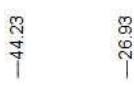

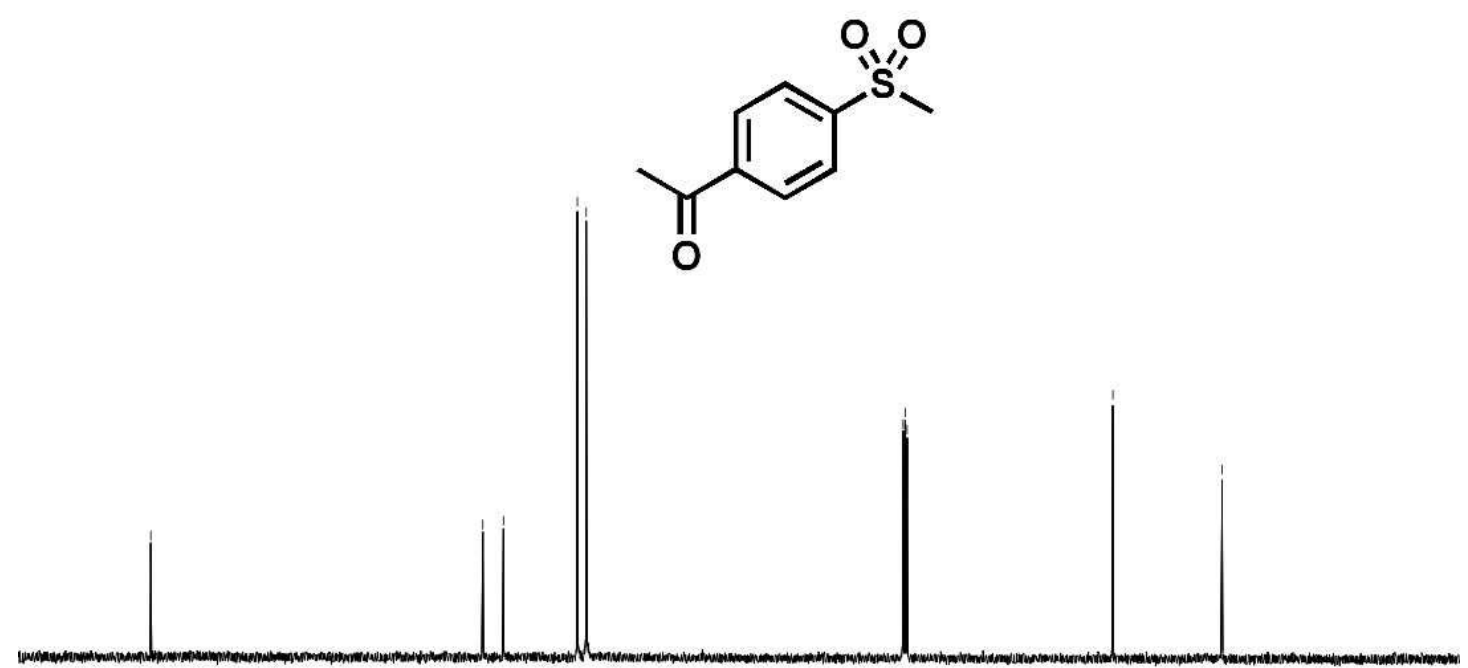

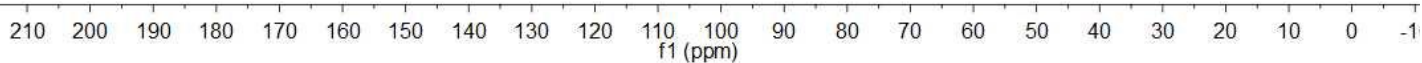


${ }^{1} \mathrm{H}$ NMR of product $2 \mathrm{p}$ in $\mathrm{CDCl}_{3}(400 \mathrm{MHz})$

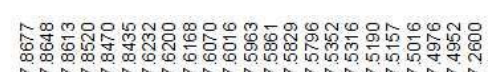

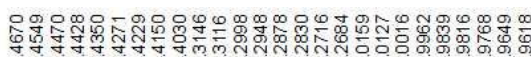

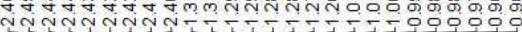

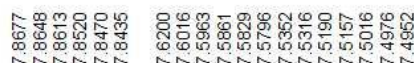

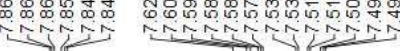
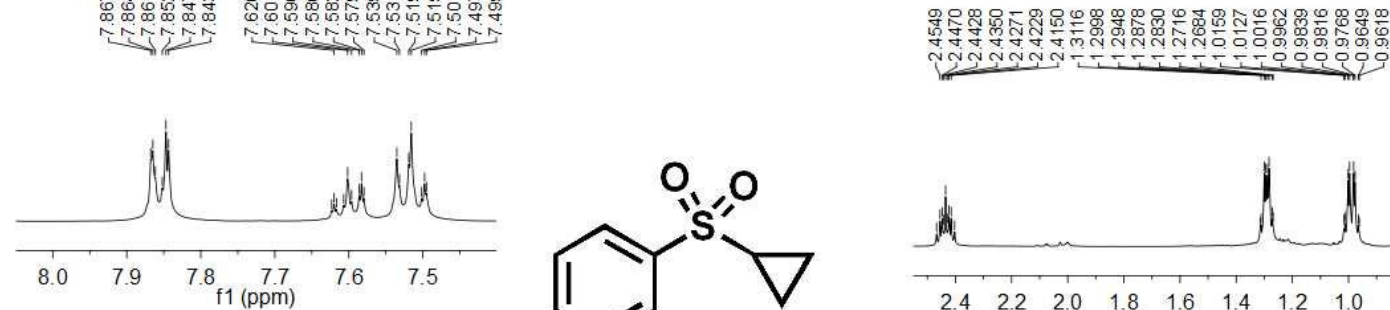<smiles>O=S(=O)(c1ccccc1)C1CC1</smiles>
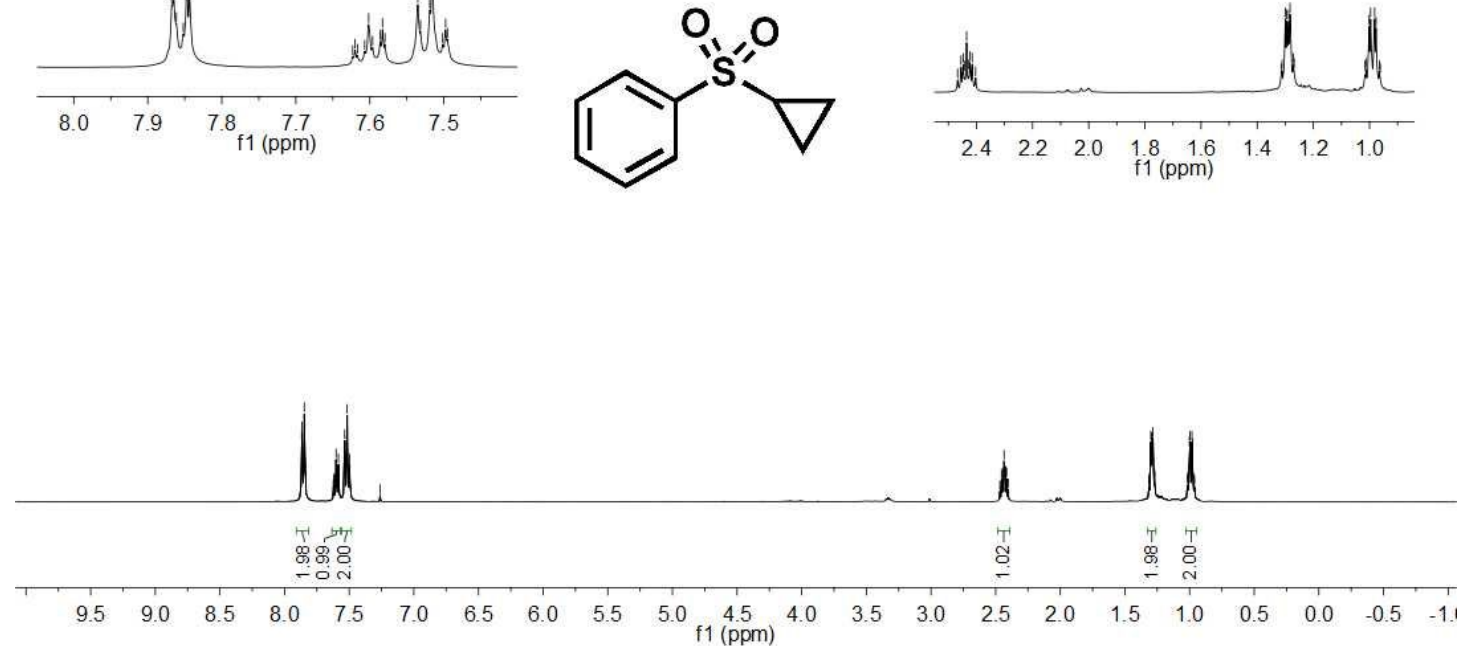

${ }^{13} \mathrm{C}$ NMR of product $2 \mathrm{p}$ in $\mathrm{CDCl}_{3}(100 \mathrm{MHz})$

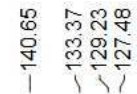

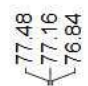

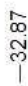

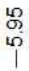

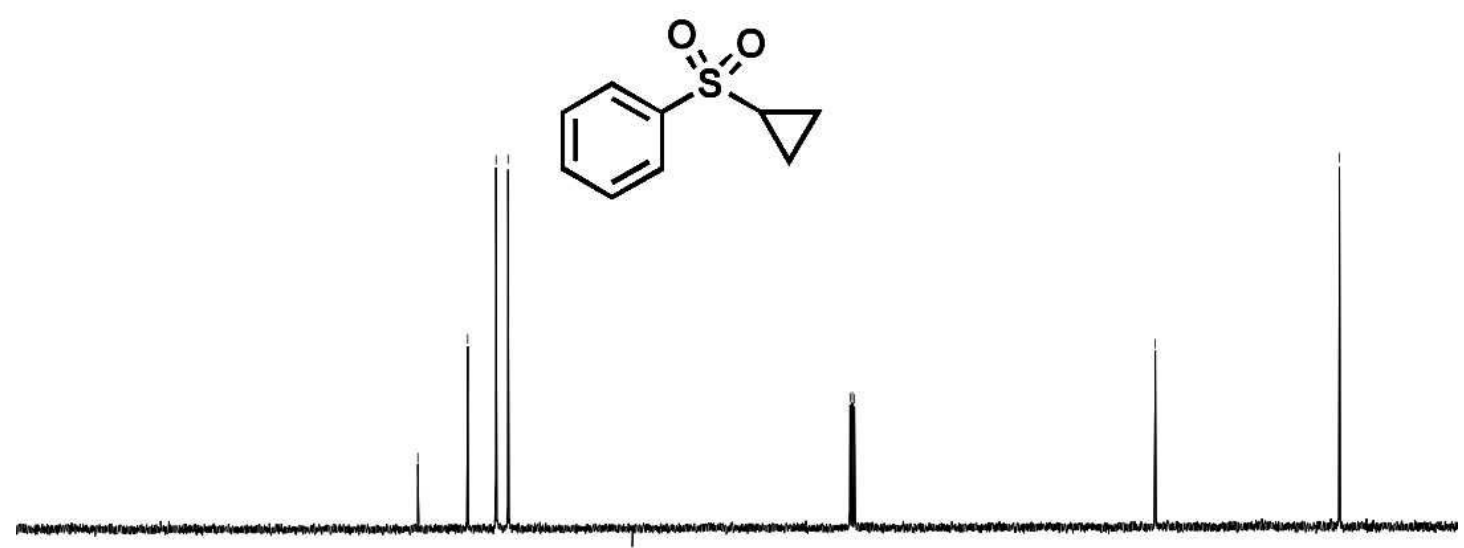

$\begin{array}{llllllllll}190 & 180 & 170 & 160 & 150 & 140 & 130 & 120 & 110 & \begin{array}{l}100 \\ \mathrm{f} 1(\mathrm{ppm})\end{array}\end{array}$

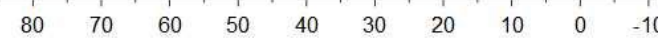


${ }^{1} \mathrm{H}$ NMR of product 2q in $\mathrm{CDCl}_{3}(400 \mathrm{MHz})$

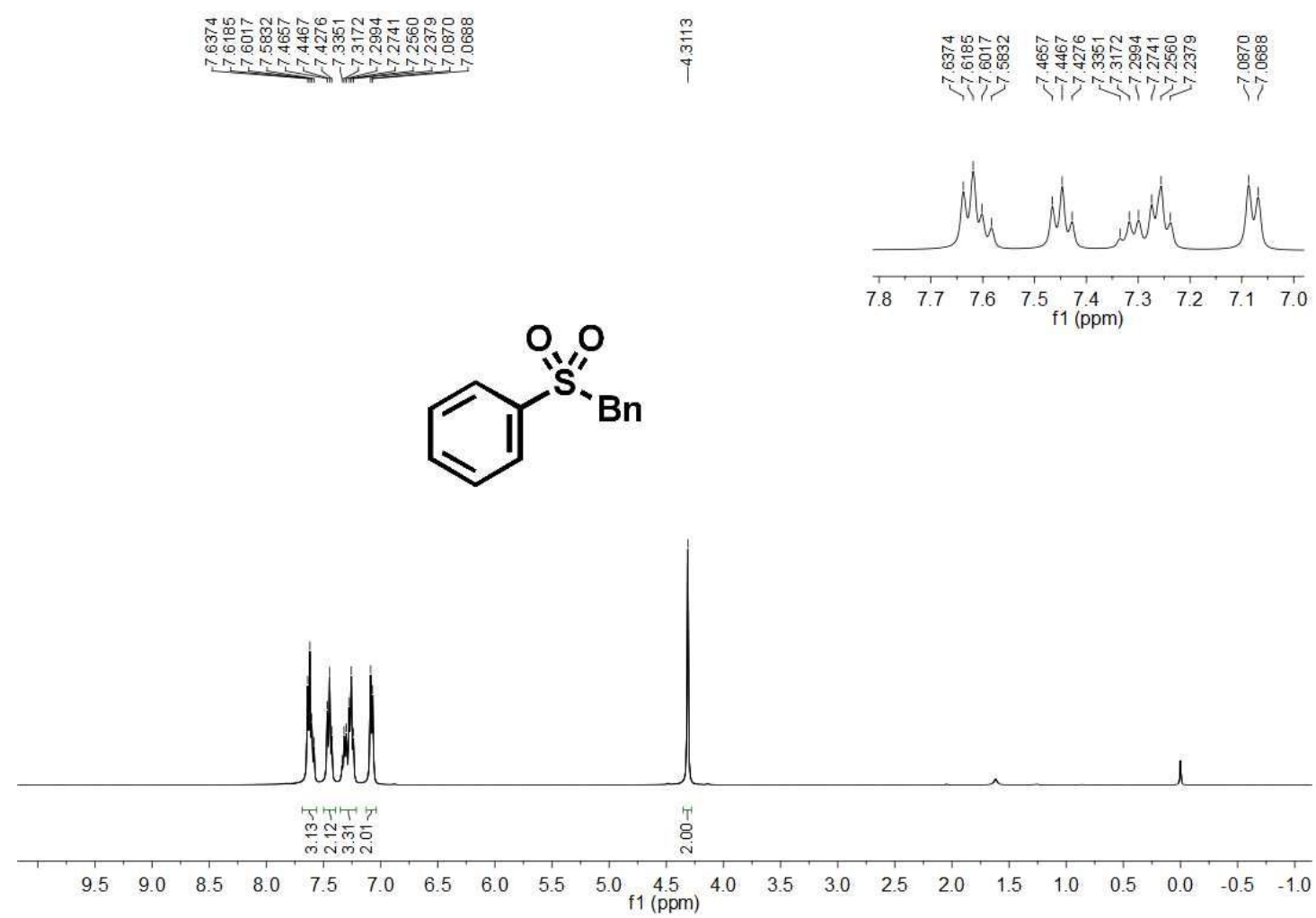

${ }^{13} \mathrm{C}$ NMR of product $2 q$ in $\mathrm{CDCl}_{3}(100 \mathrm{MHz})$

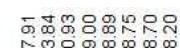

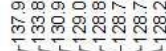

紫采

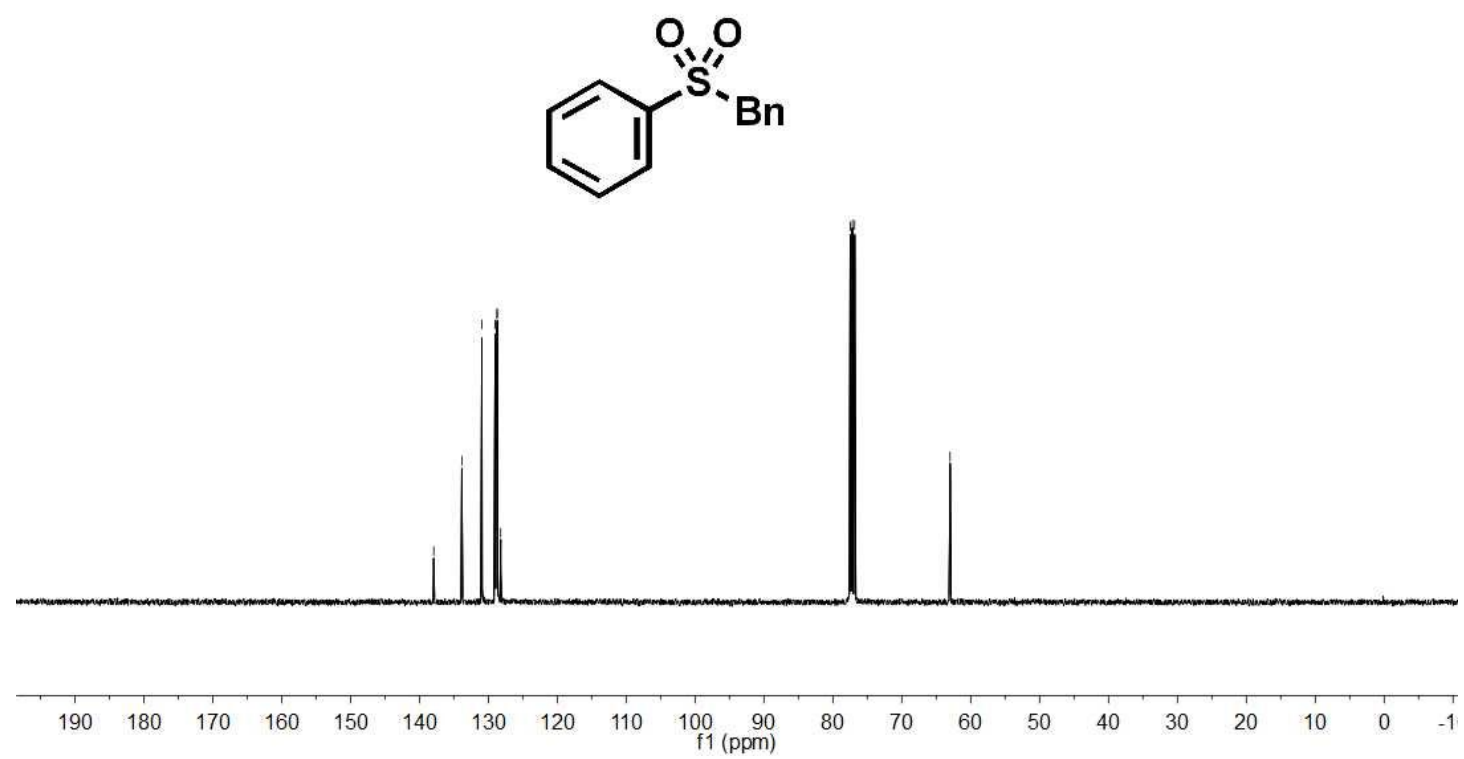


${ }^{1} \mathrm{H}$ NMR of product $2 \mathrm{r}$ in $\mathrm{CDCl}_{3}(400 \mathrm{MHz})$

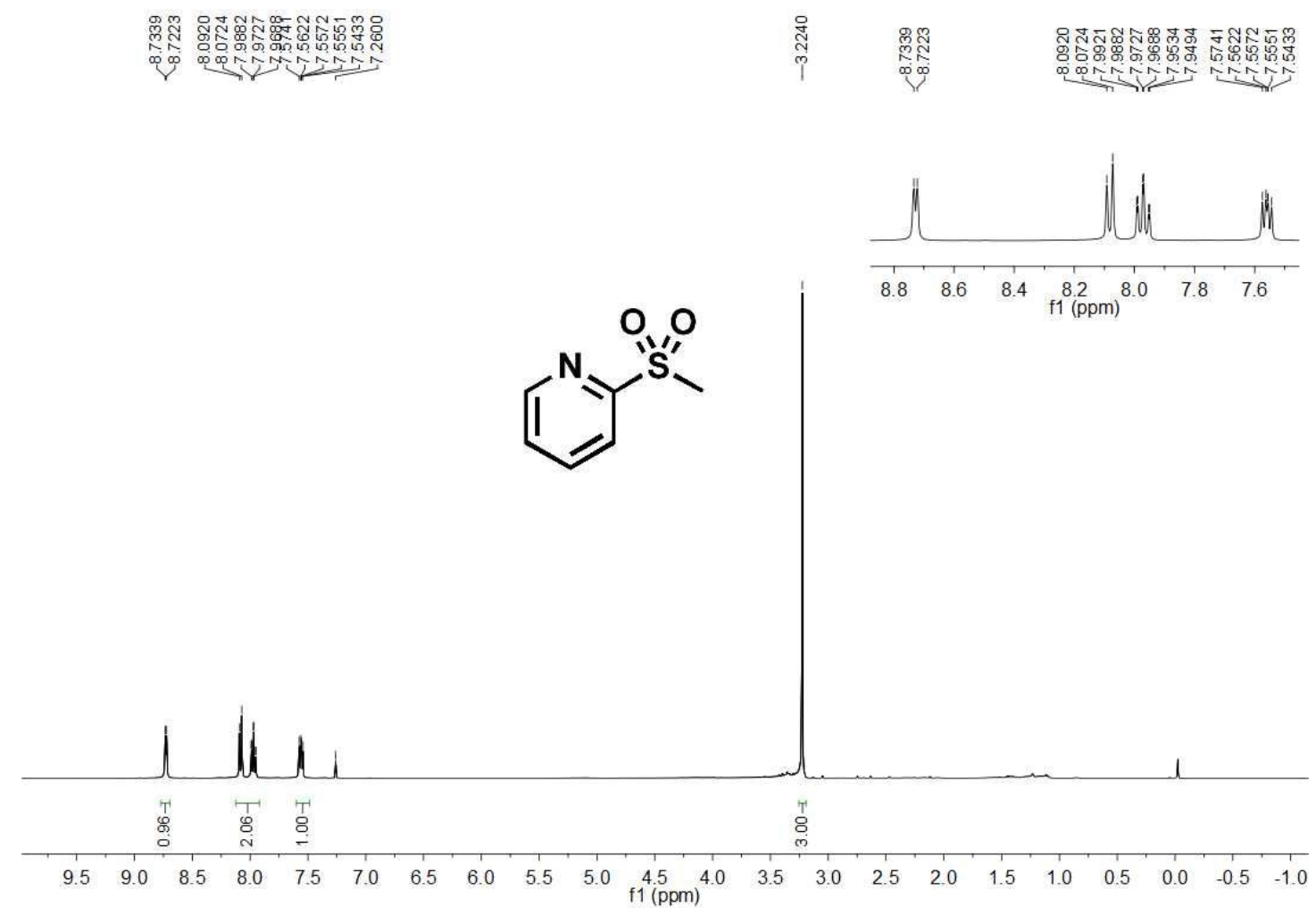

${ }^{13} \mathrm{C}$ NMR of product $2 \mathrm{r}$ in $\mathrm{CDCl}_{3}(100 \mathrm{MHz})$

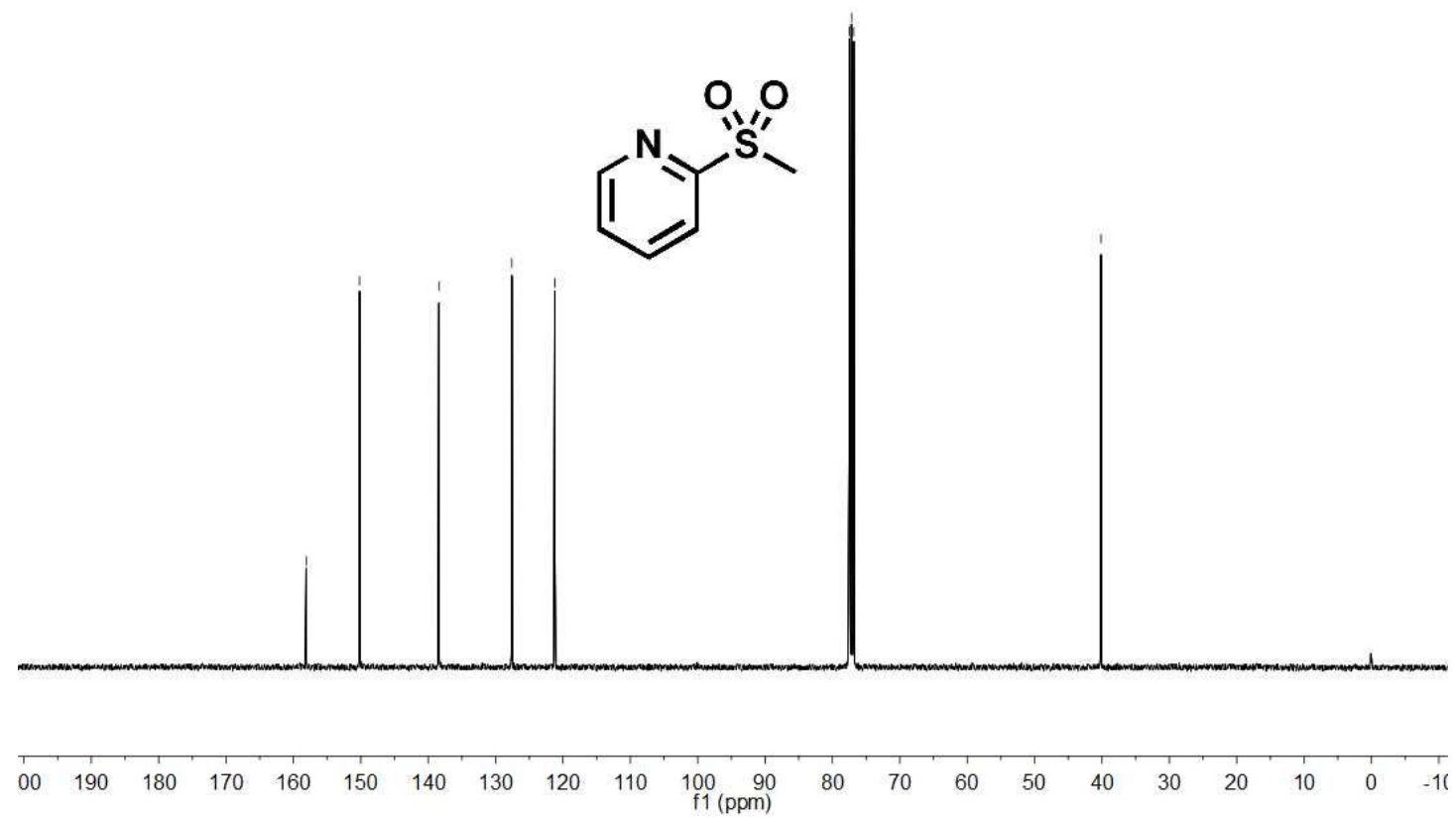


${ }^{1} \mathrm{H}$ NMR of product $2 \mathrm{~s}$ in $\mathrm{CDCl}_{3}(400 \mathrm{MHz})$

용<smiles>CS(=O)(=O)c1nc(Cl)cc(Cl)n1</smiles>

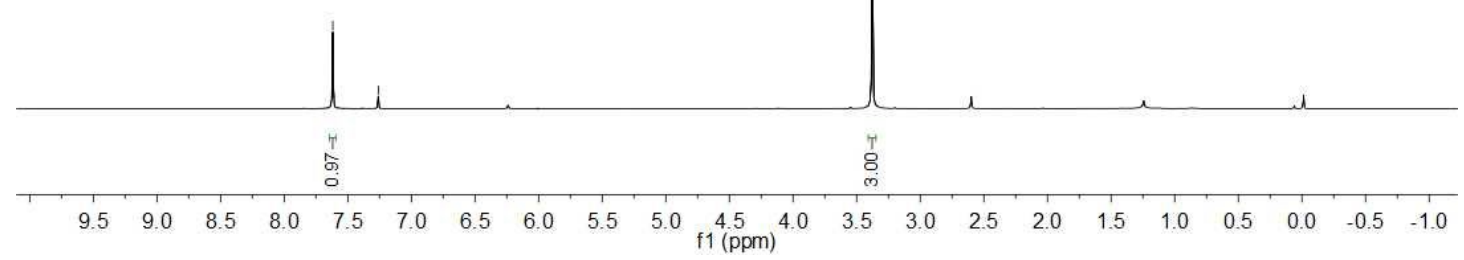

${ }^{13} \mathrm{C}$ NMR of product $2 \mathrm{~s}$ in $\mathrm{CDCl}_{3}(100 \mathrm{MHz})$

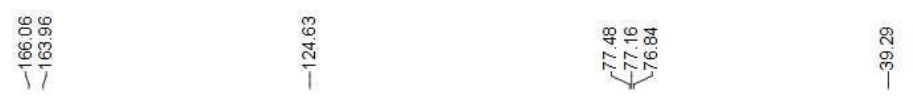

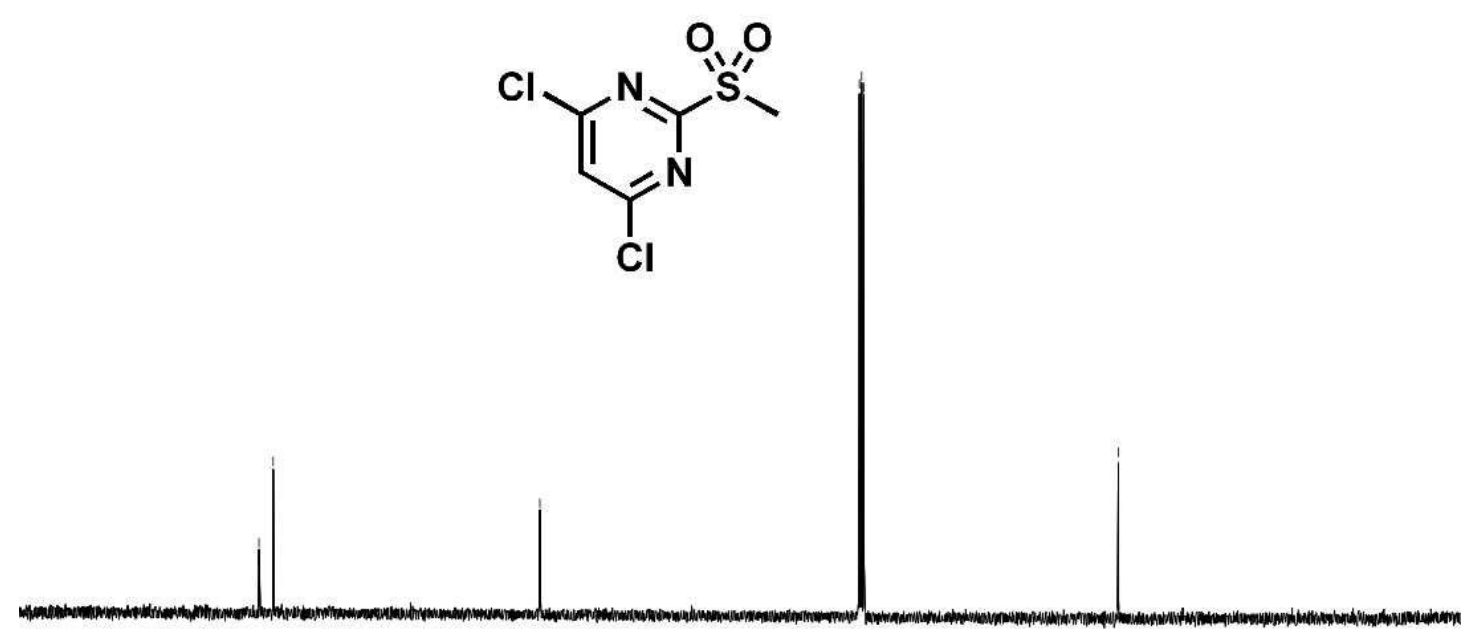

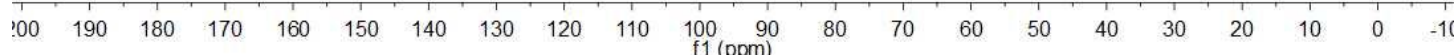


${ }^{1} \mathrm{H}$ NMR of product $3 \mathrm{a}$ in $\mathrm{CDCl}_{3}(400 \mathrm{MHz})$
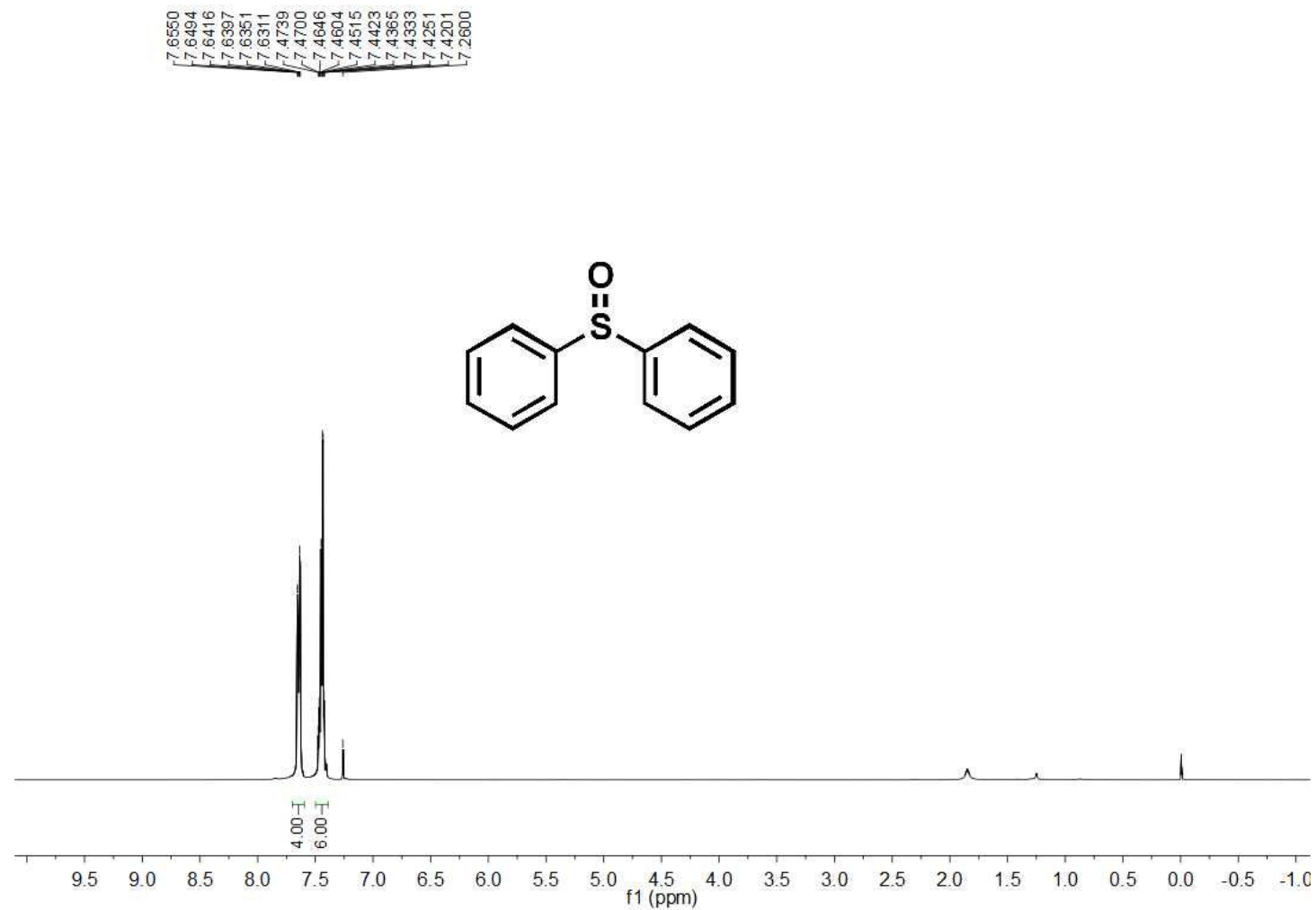

${ }^{13} \mathrm{C}$ NMR of product $3 \mathrm{a}$ in $\mathrm{CDCl}_{3}(100 \mathrm{MHz})$

广

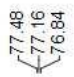

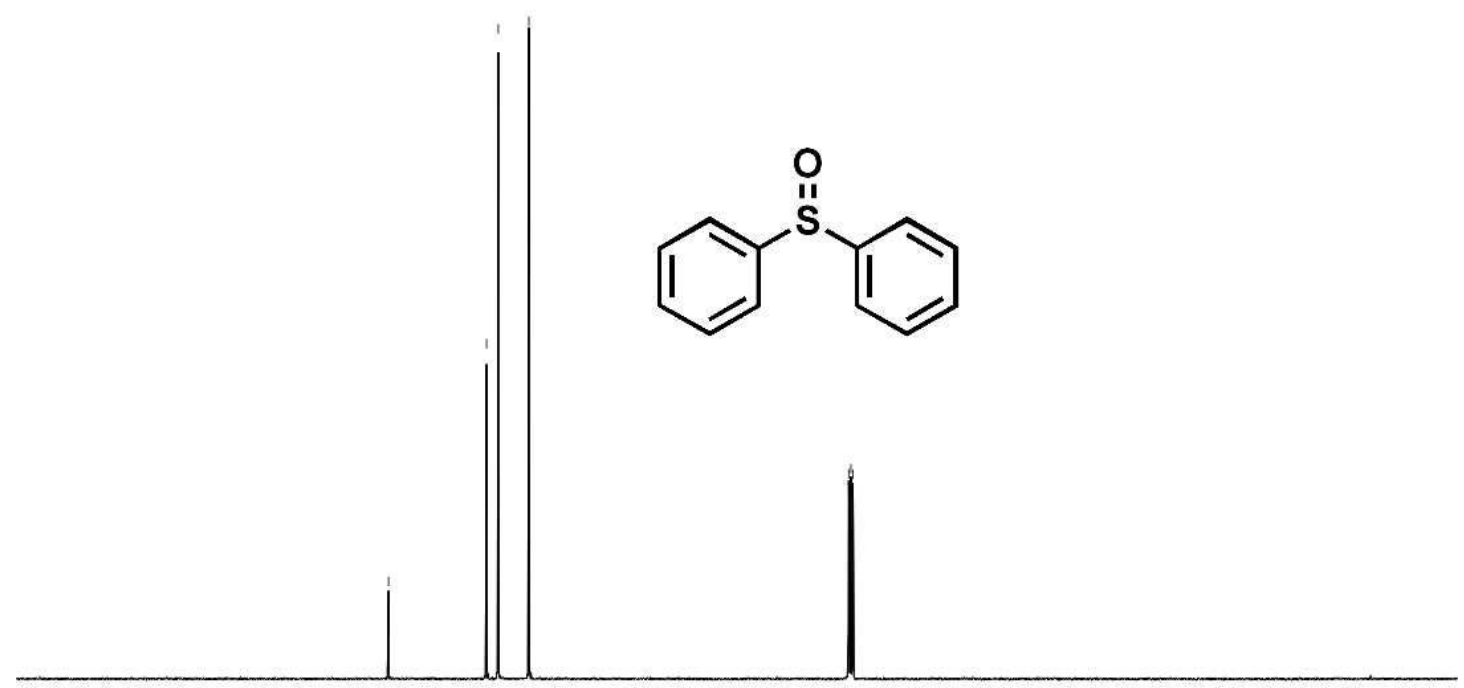

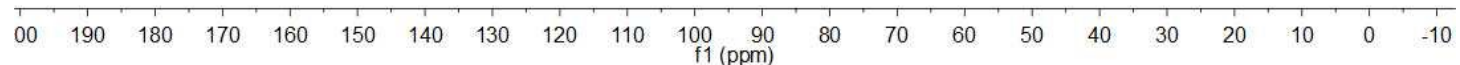


${ }^{1} \mathrm{H}$ NMR of product $3 \mathrm{~b}$ in $\mathrm{CDCl}_{3}(400 \mathrm{MHz})$

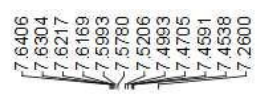<smiles>O=S(c1ccccc1)c1ccc(Br)cc1</smiles>
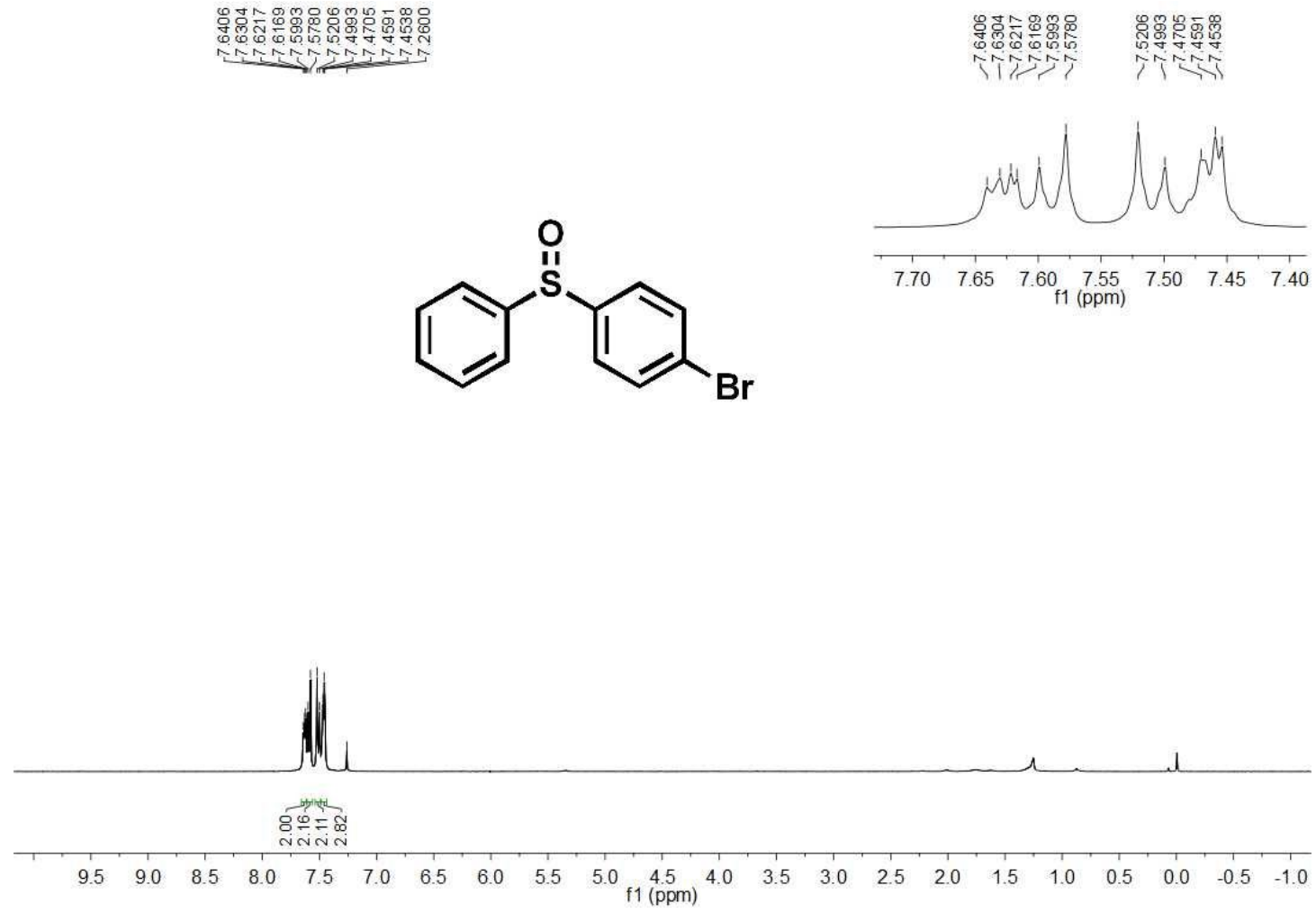

${ }^{13} \mathrm{C}$ NMR of product $3 \mathrm{~b}$ in $\mathrm{CDCl}_{3}(100 \mathrm{MHz})$

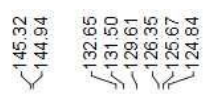<smiles>O=S(c1ccccc1)c1ccc(Br)cc1</smiles>

$\begin{array}{lllllllllll}00 & 190 & 180 & 170 & 160 & 150 & 140 & 130 & 120 & 110 & \begin{array}{r}100 \\ \mathrm{f} 1(\mathrm{ppm})\end{array}\end{array}$ 
${ }^{1} \mathrm{H}$ NMR of product $3 \mathrm{c}$ in $\mathrm{CDCl}_{3}(400 \mathrm{MHz})$

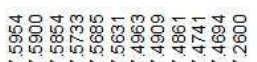

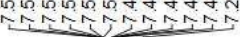<smiles>O=S(c1ccc(Br)cc1)c1ccc(Br)cc1</smiles>

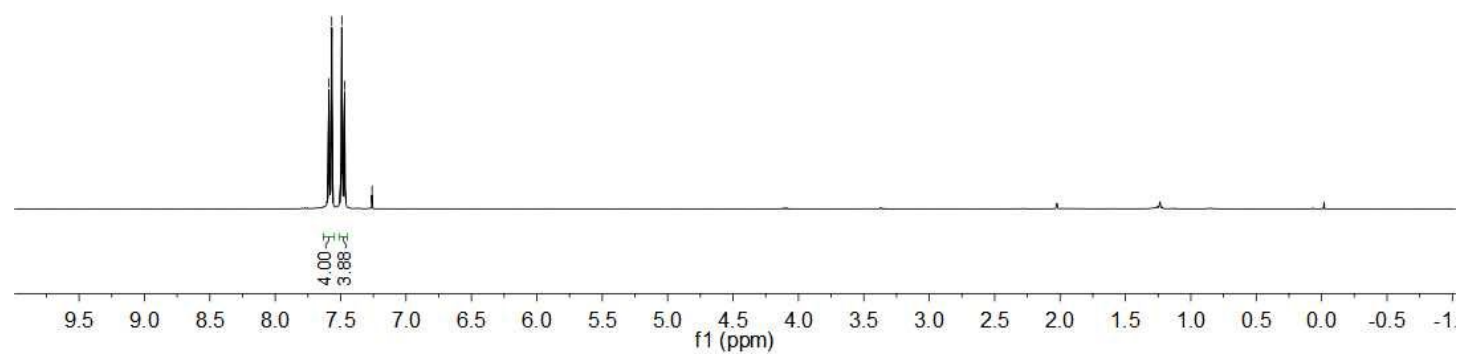

${ }^{13} \mathrm{C}$ NMR of product $3 \mathrm{c}$ in $\mathrm{CDCl}_{3}(100 \mathrm{MHz})$

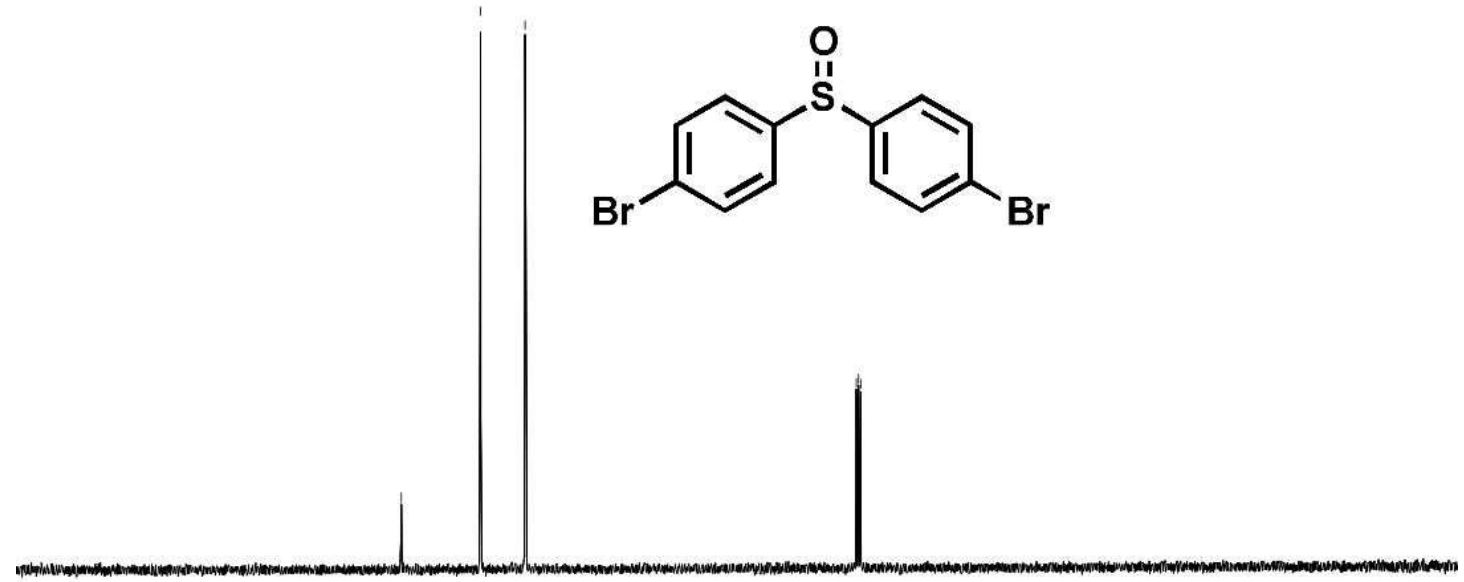

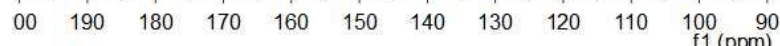

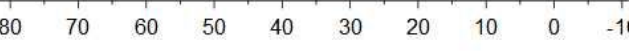


${ }^{1} \mathrm{H}$ NMR of product $3 \mathrm{~d}$ in $\mathrm{CDCl}_{3}(400 \mathrm{MHz})$

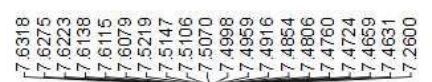

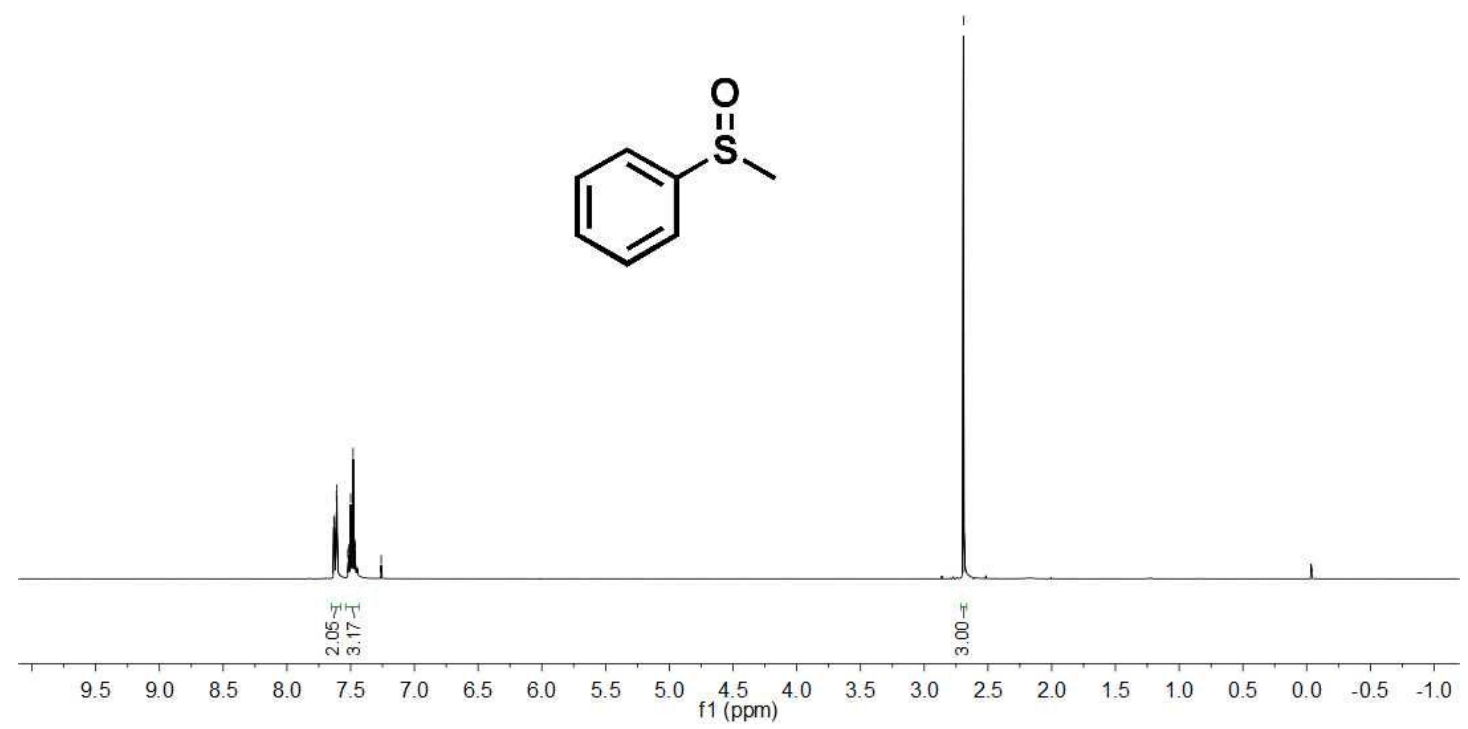

${ }^{13} \mathrm{C}$ NMR of product 3d in $\mathrm{CDCl}_{3}(100 \mathrm{MHz})$

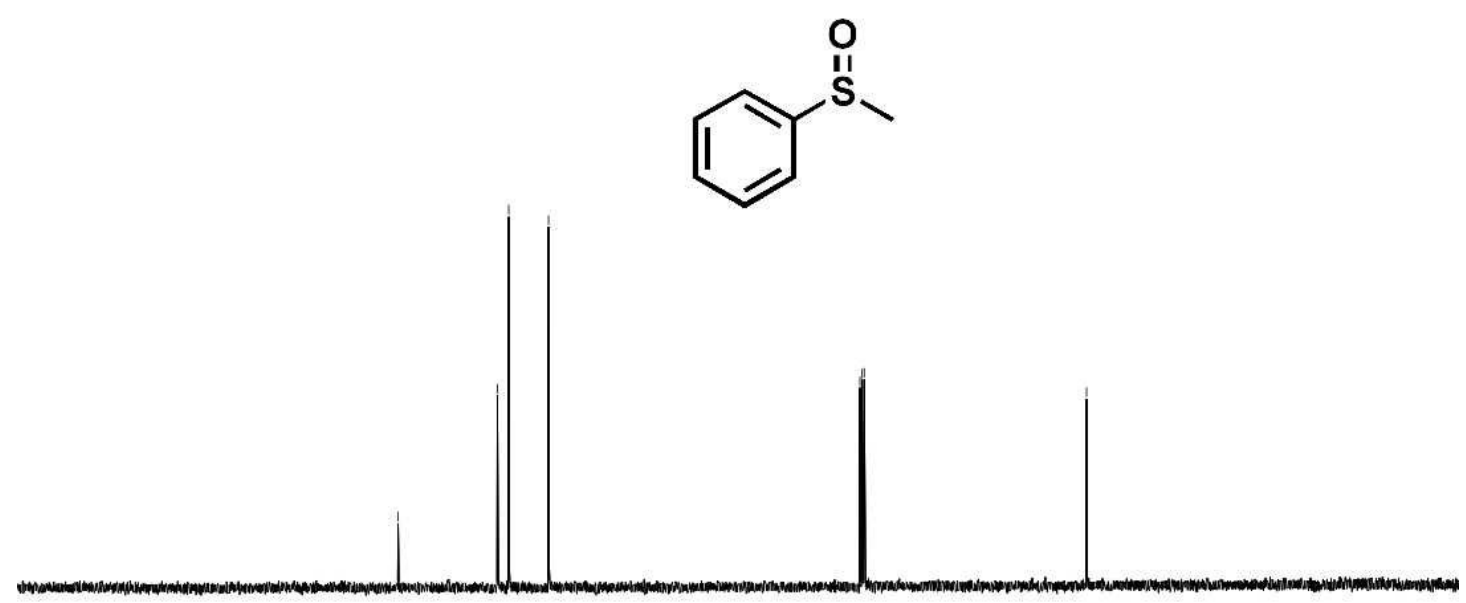

$\begin{array}{lllllllllllllllllllllll}200 & 190 & 180 & 170 & 160 & 150 & 140 & 130 & 120 & 110 & \begin{array}{l}100 \\ \mathrm{f} 1(\mathrm{ppm})\end{array} & 80 & 70 & 60 & 50 & 40 & 30 & 20 & 10 & 0 & -11\end{array}$ 
${ }^{1} \mathrm{H}$ NMR of product $3 \mathrm{e}$ in $\mathrm{CDCl}_{3}(400 \mathrm{MHz})$

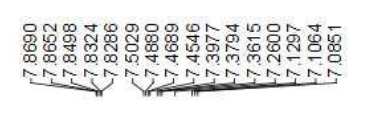

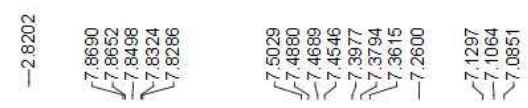<smiles>CS(=O)c1ccccc1F</smiles>
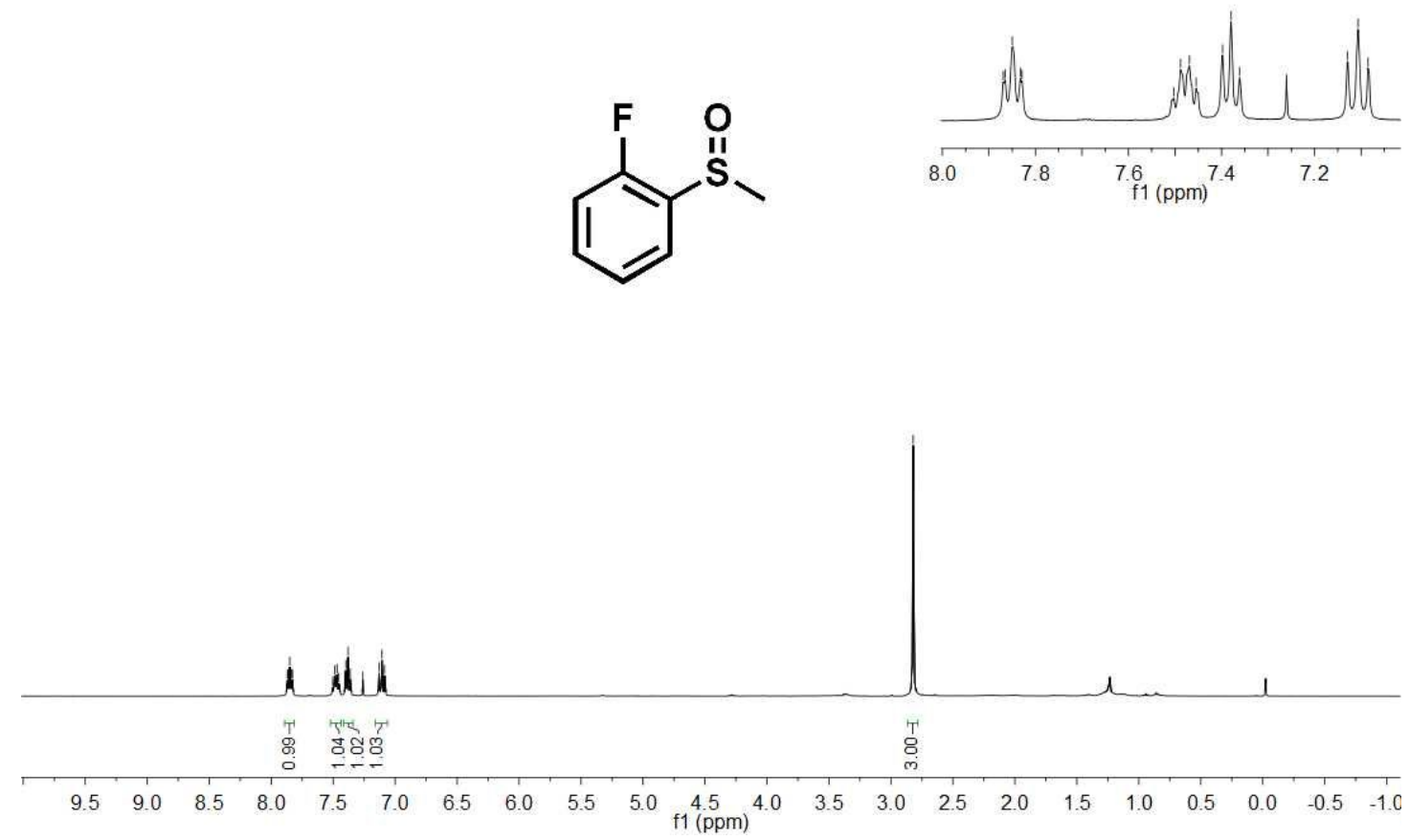

${ }^{13} \mathrm{C}$ NMR of product $3 \mathrm{e}$ in $\mathrm{CDCl}_{3}(100 \mathrm{MHz})$

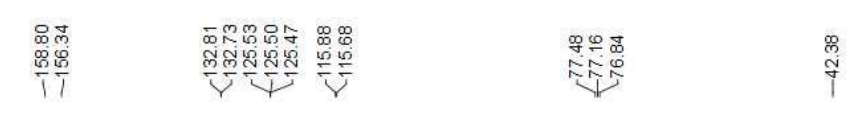

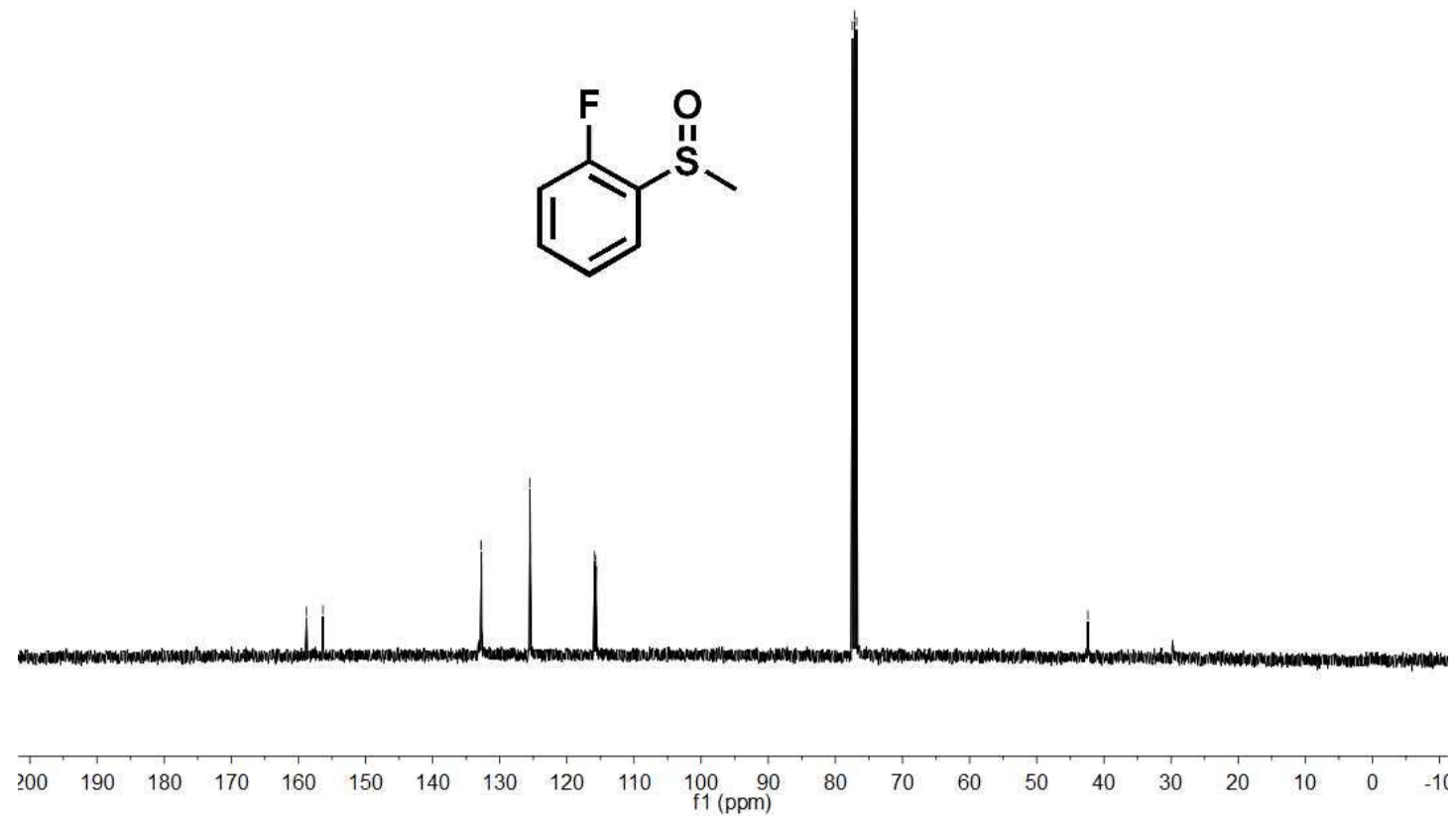


${ }^{1} \mathrm{H}$ NMR of product $3 \mathrm{f}$ in $\mathrm{CDCl}_{3}(400 \mathrm{MHz})$

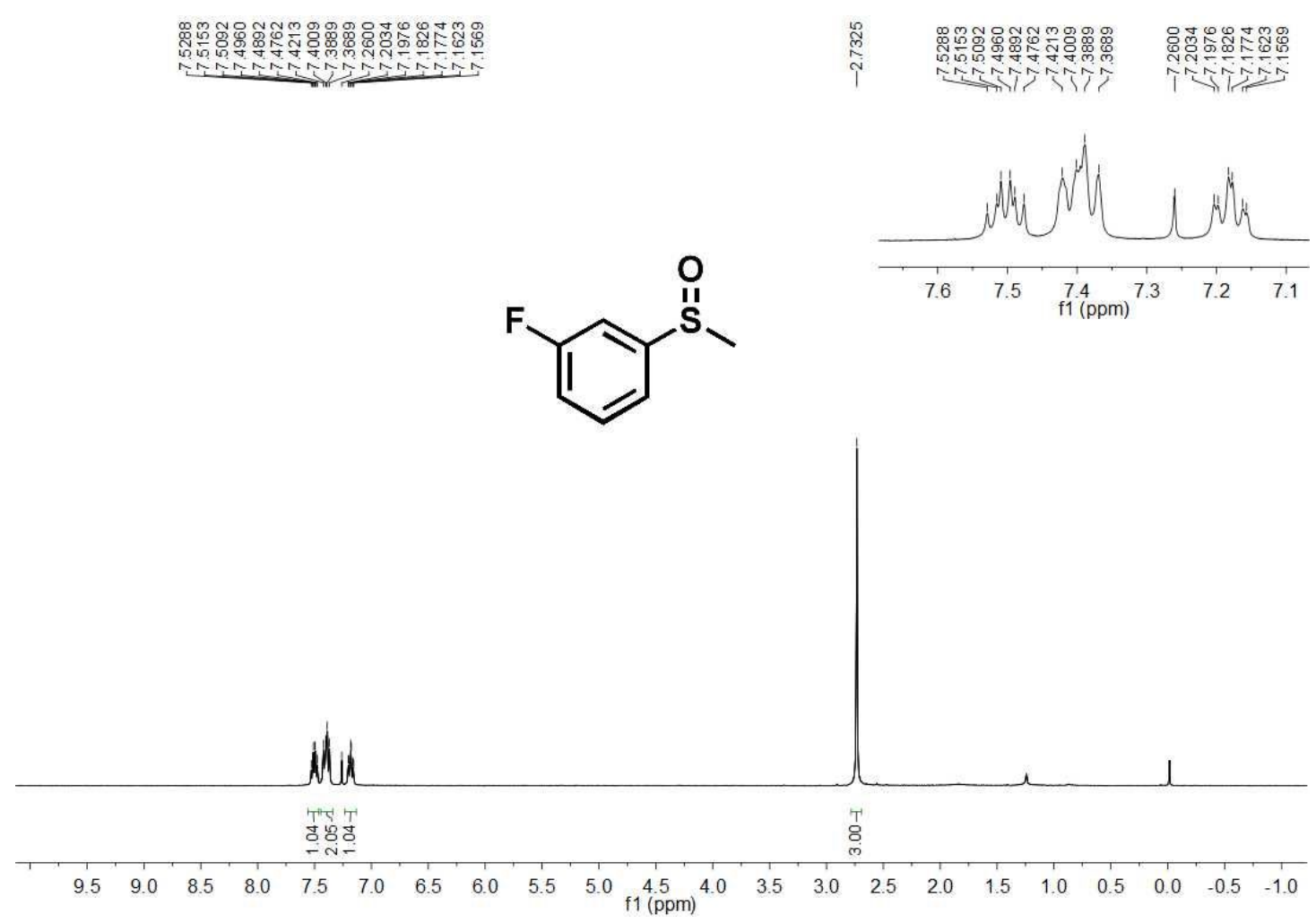

${ }^{13} \mathrm{C}$ NMR of product $3 \mathrm{f}$ in $\mathrm{CDCl}_{3}(100 \mathrm{MHz})$

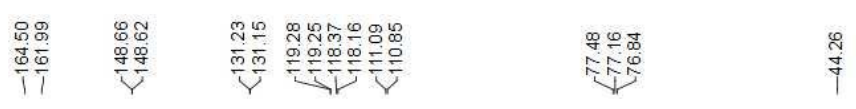<smiles>CS(=O)c1cccc(F)c1</smiles>

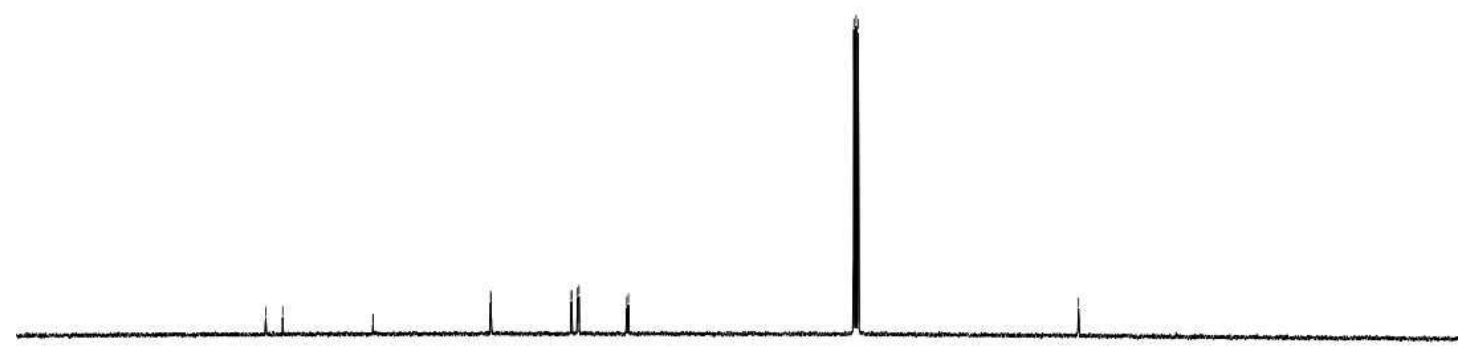

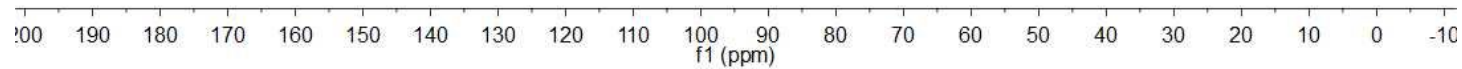


${ }^{1} \mathrm{H}$ NMR of product $3 \mathrm{~g}$ in $\mathrm{CDCl}_{3}(400 \mathrm{MHz})$
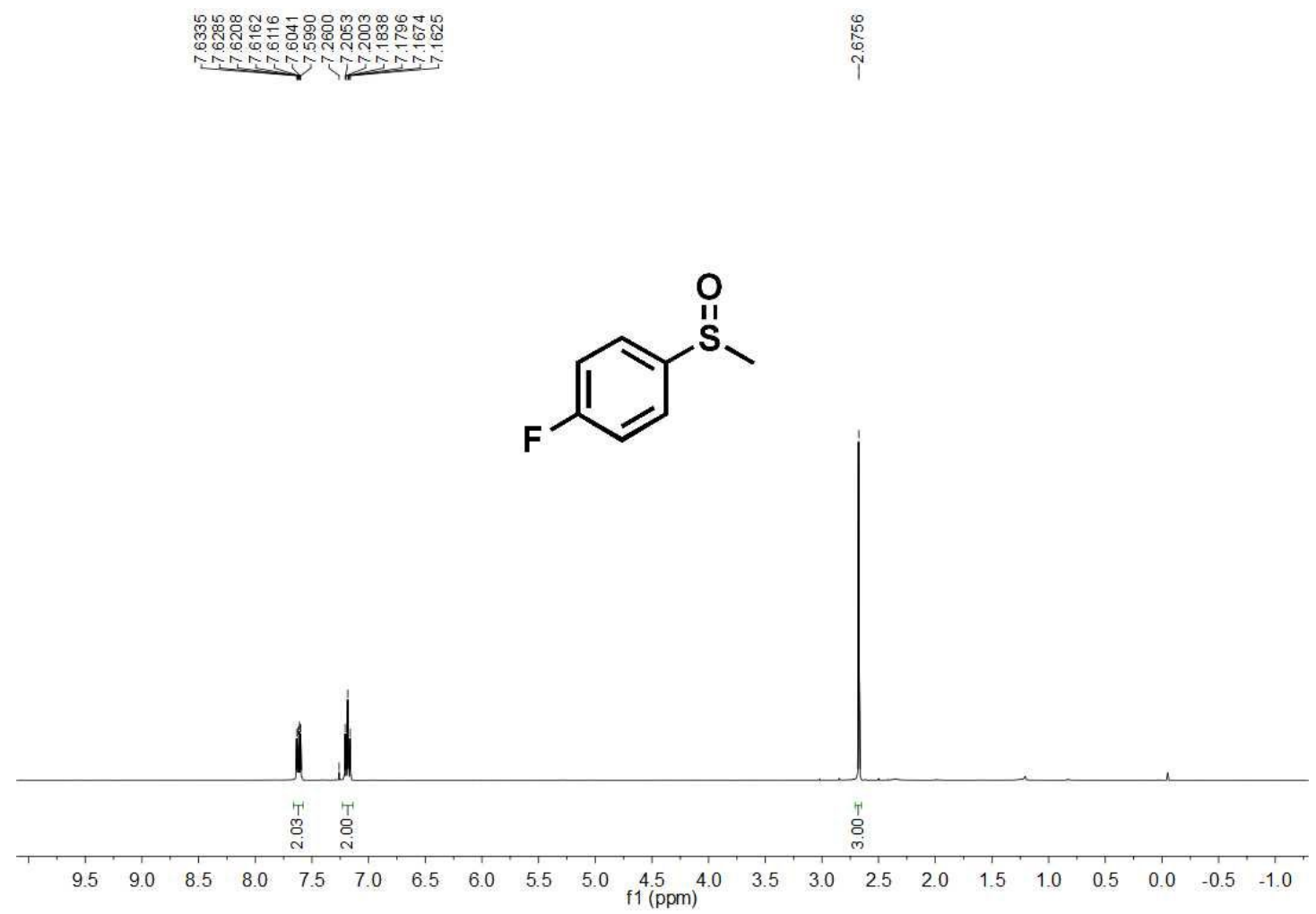

${ }^{13} \mathrm{C}$ NMR of product $3 \mathrm{~g}$ in $\mathrm{CDCl}_{3}(100 \mathrm{MHz})$

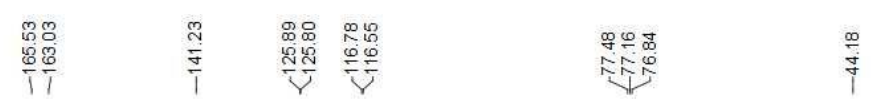

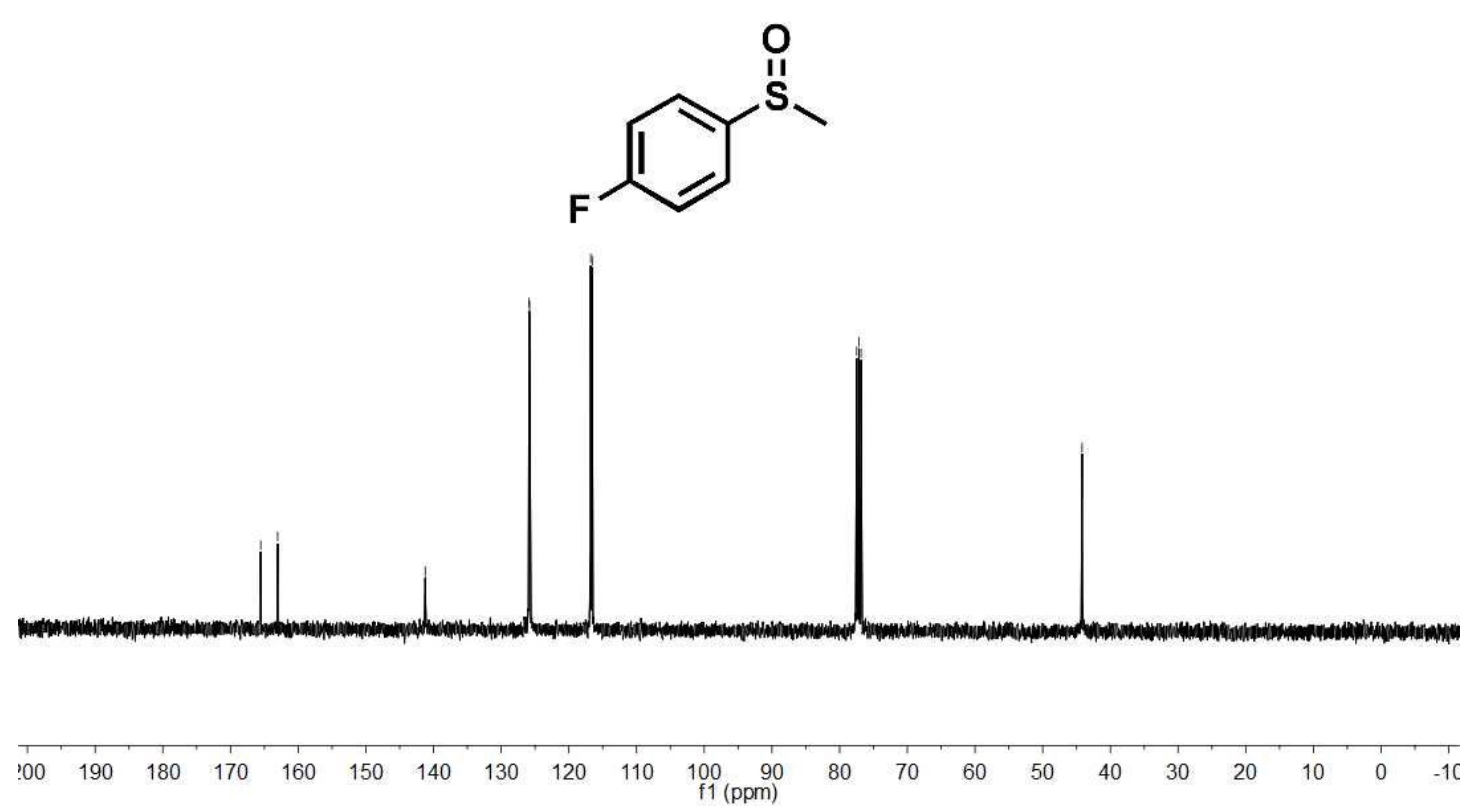


${ }^{1} \mathrm{H}$ NMR of product $3 \mathrm{~h}$ in $\mathrm{CDCl}_{3}(400 \mathrm{MHz})$

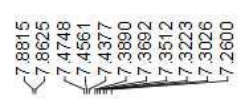

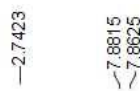

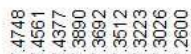

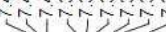

S's
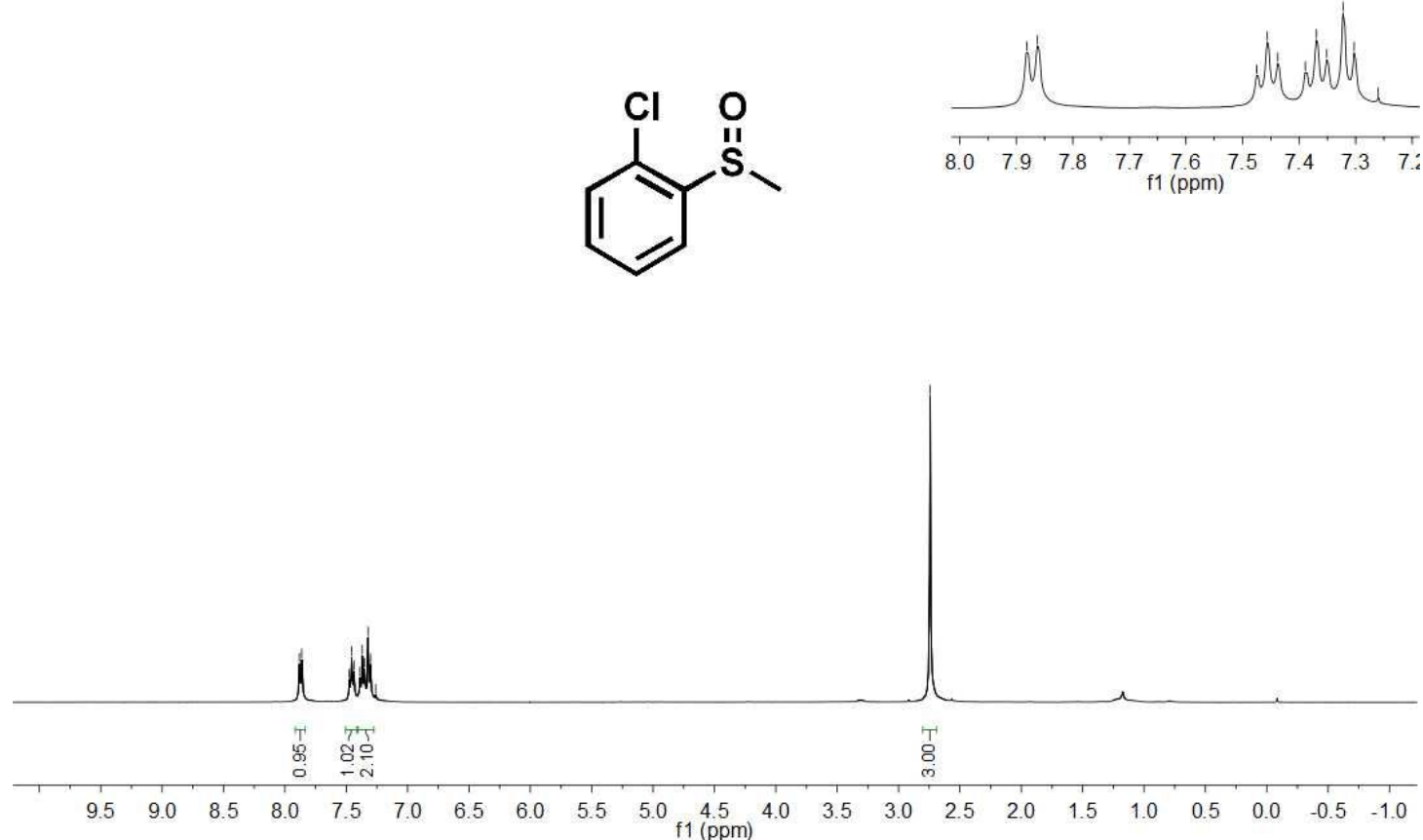

${ }^{13} \mathrm{C}$ NMR of product $3 \mathrm{~h}$ in $\mathrm{CDCl}_{3}(100 \mathrm{MHz})$

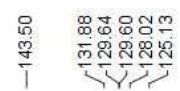

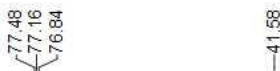<smiles>CS(=O)c1ccccc1Cl</smiles>

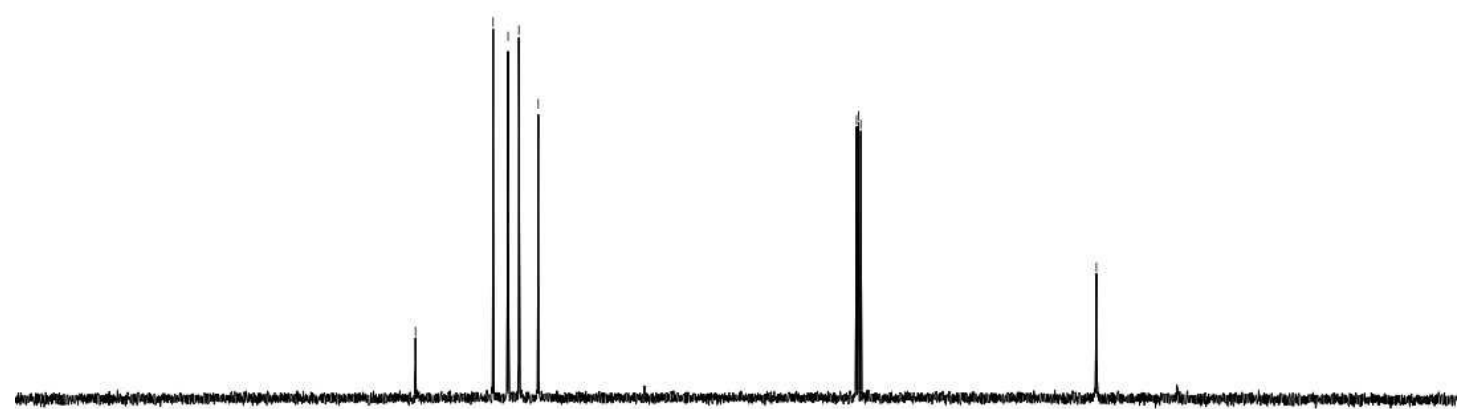

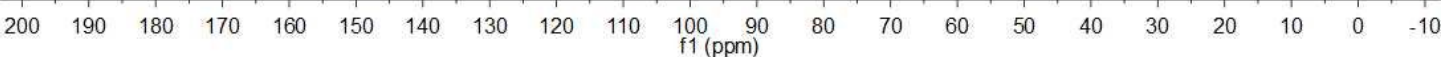


${ }^{1} \mathrm{H}$ NMR of product $3 \mathrm{i}$ in $\mathrm{CDCl}_{3}(400 \mathrm{MHz})$

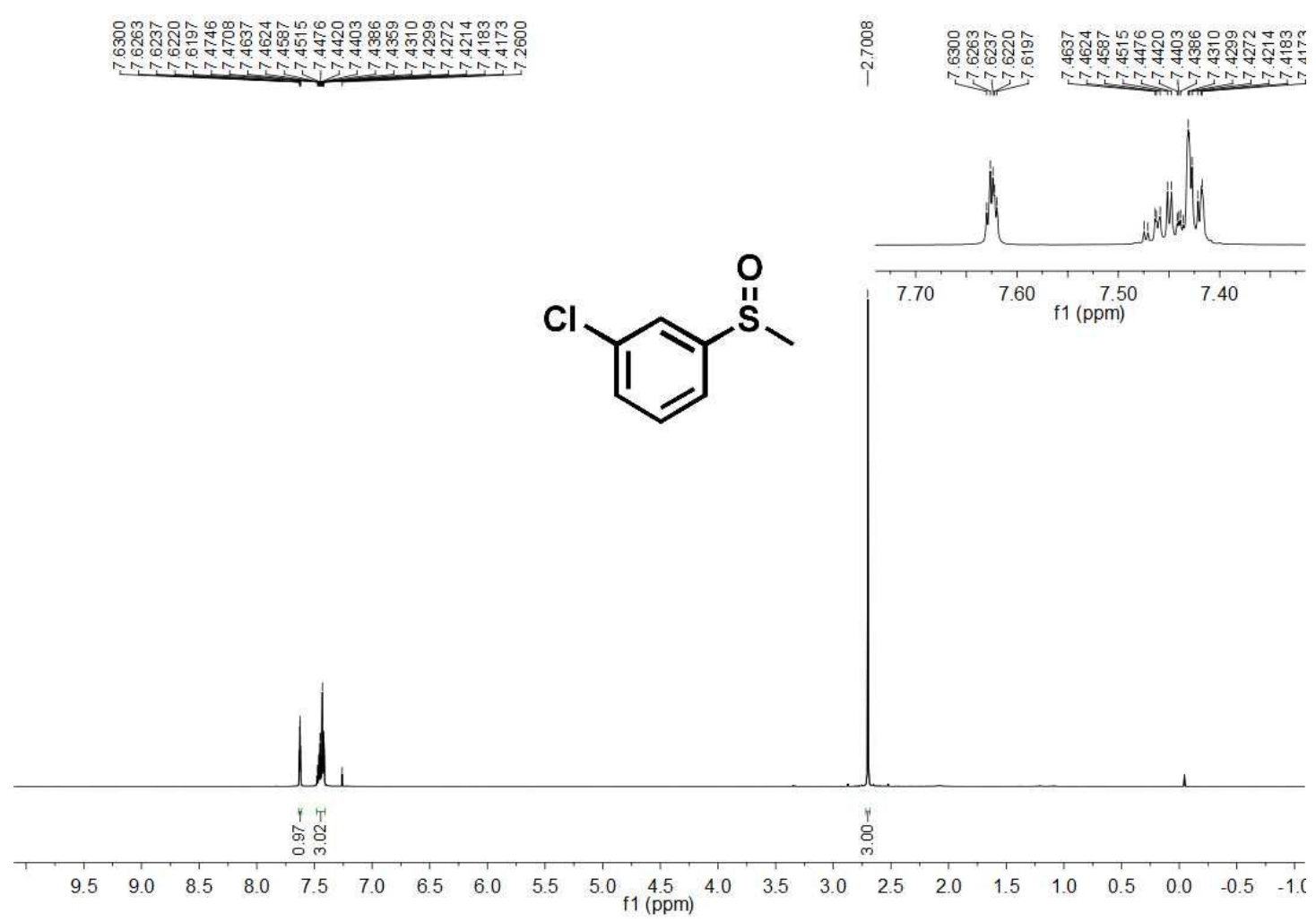

${ }^{13} \mathrm{C}$ NMR of product $3 \mathrm{i}$ in $\mathrm{CDCl}_{3}(100 \mathrm{MHz})$

\begin{tabular}{|c|c|}
\hline 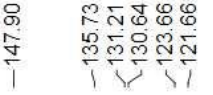 & 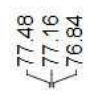 \\
\hline
\end{tabular}<smiles>CS(=O)c1cccc(Cl)c1</smiles>

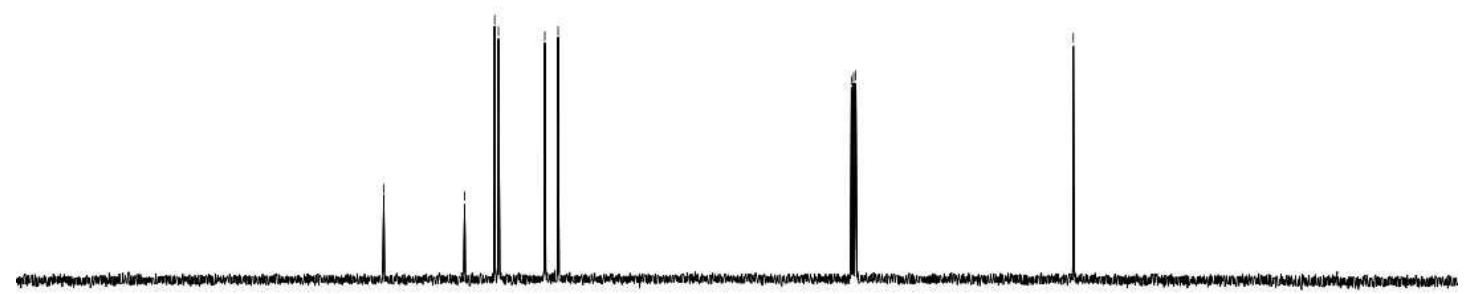

\begin{tabular}{lllllllllll}
200 & 190 & 180 & 170 & 160 & 150 & 140 & 130 & 120 & 110 & $\begin{array}{c}100 \\
\mathrm{f} 1(\mathrm{ppm})\end{array}$ \\
\hline
\end{tabular} 
${ }^{1} \mathrm{H}$ NMR of product $3 \mathrm{j}$ in $\mathrm{CDCl}_{3}(400 \mathrm{MHz})$
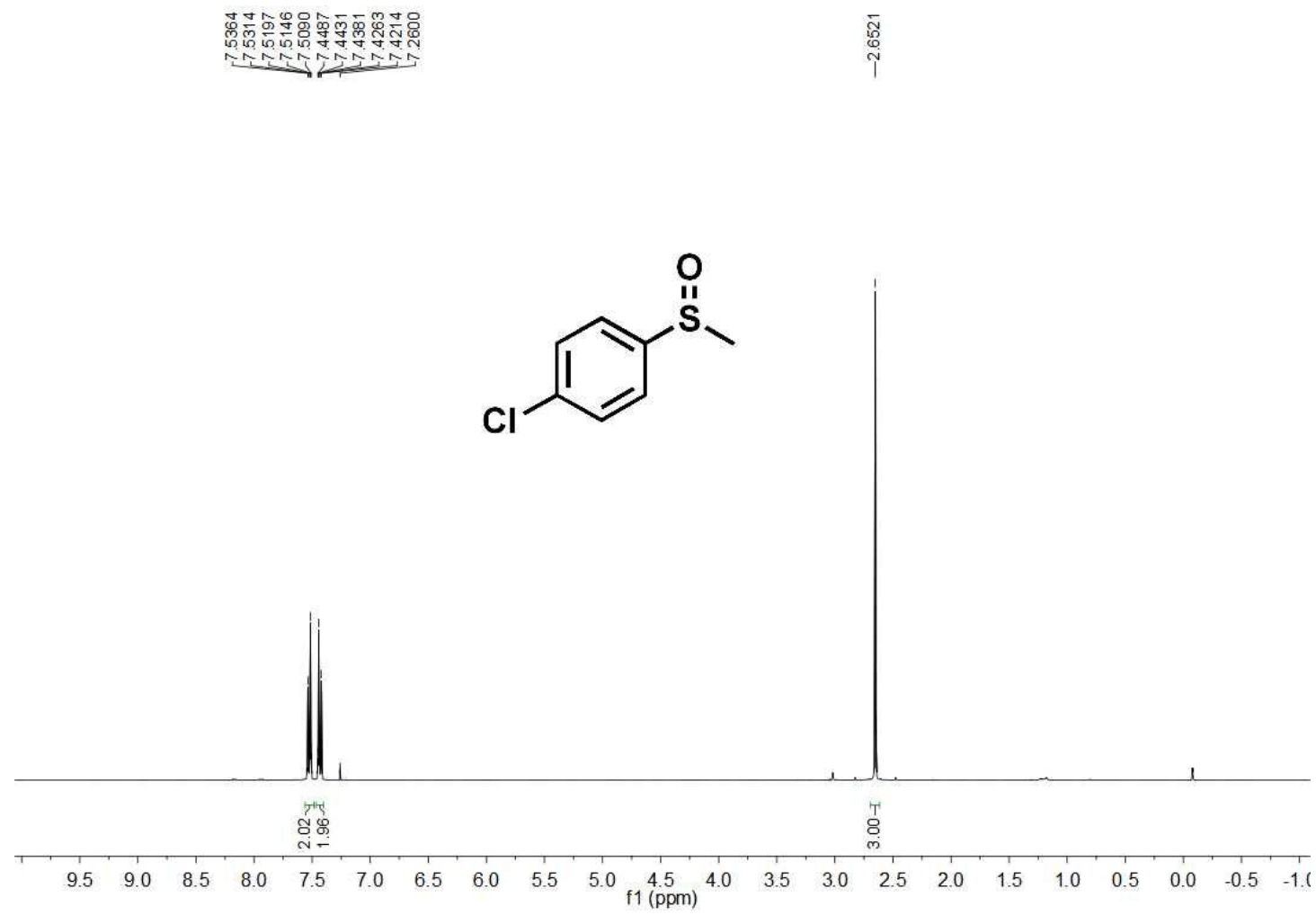

${ }^{13} \mathrm{C}$ NMR of product $3 \mathrm{j}$ in $\mathrm{CDCl}_{3}(100 \mathrm{MHz})$

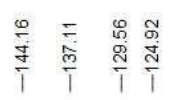

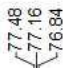

के

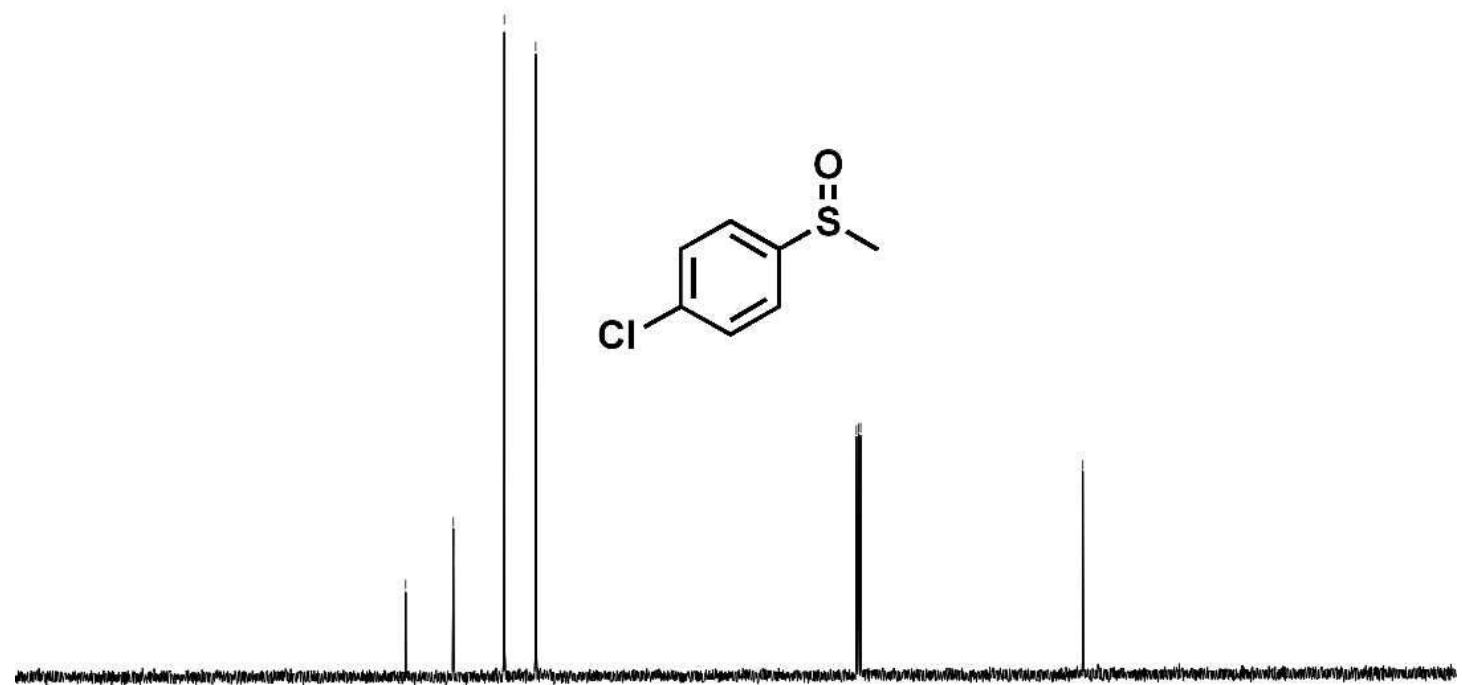

$\begin{array}{lllllllllllllllllllll}200 & 190 & 180 & 170 & 160 & 150 & 140 & 130 & 120 & 110 & \underset{f 1}{100}(\mathrm{ppm}) & 80 & 70 & 60 & 50 & 40 & 30 & 20 & 10 & 0 & -1(\end{array}$ 
${ }^{1} \mathrm{H}$ NMR of product $3 \mathrm{k}$ in $\mathrm{CDCl}_{3}(400 \mathrm{MHz})$

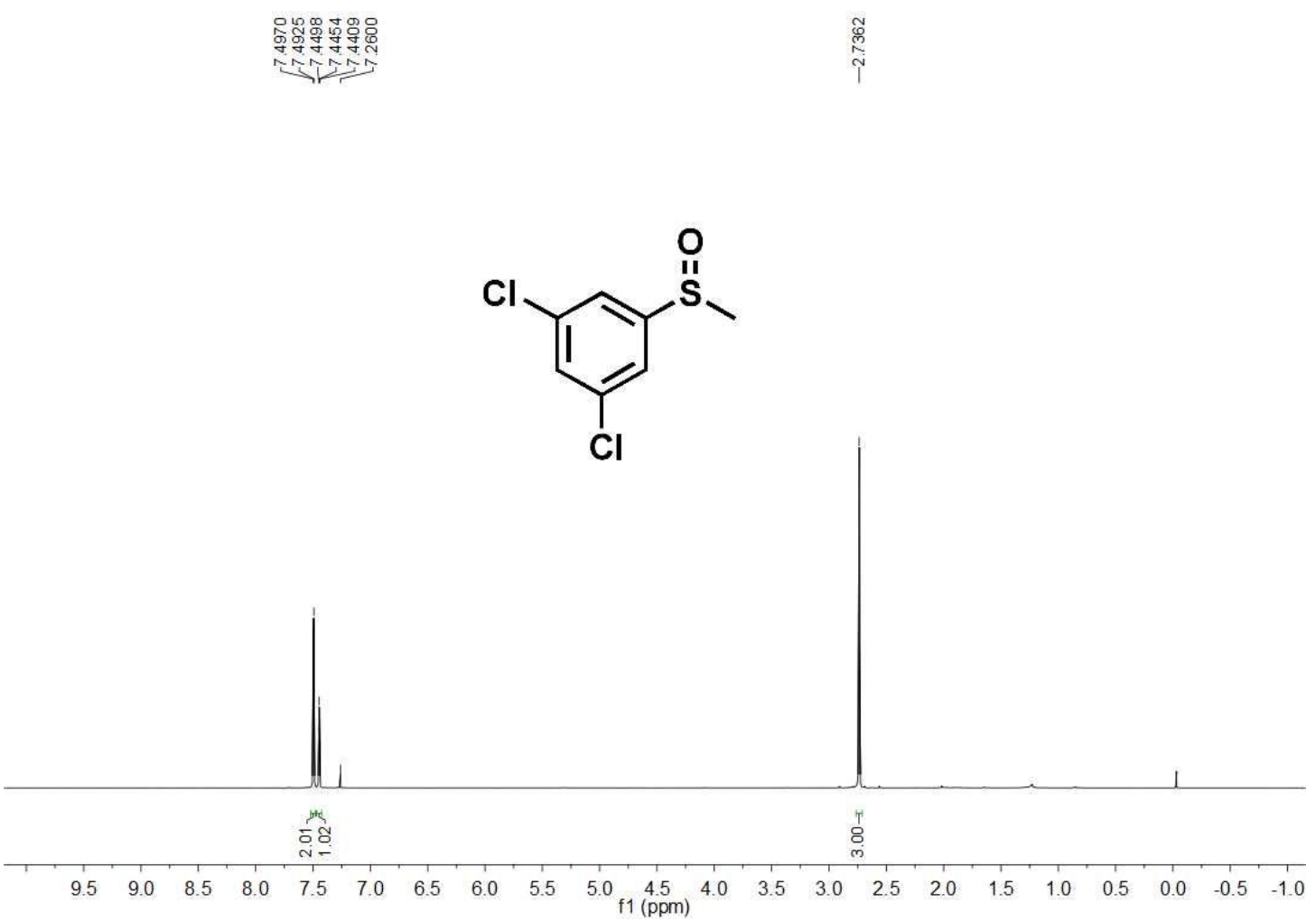

${ }^{13} \mathrm{C}$ NMR of product $3 \mathrm{k}$ in $\mathrm{CDCl}_{3}(100 \mathrm{MHz})$

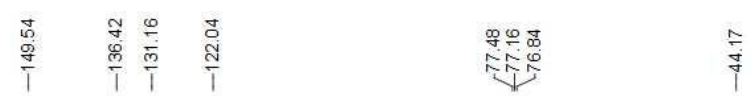

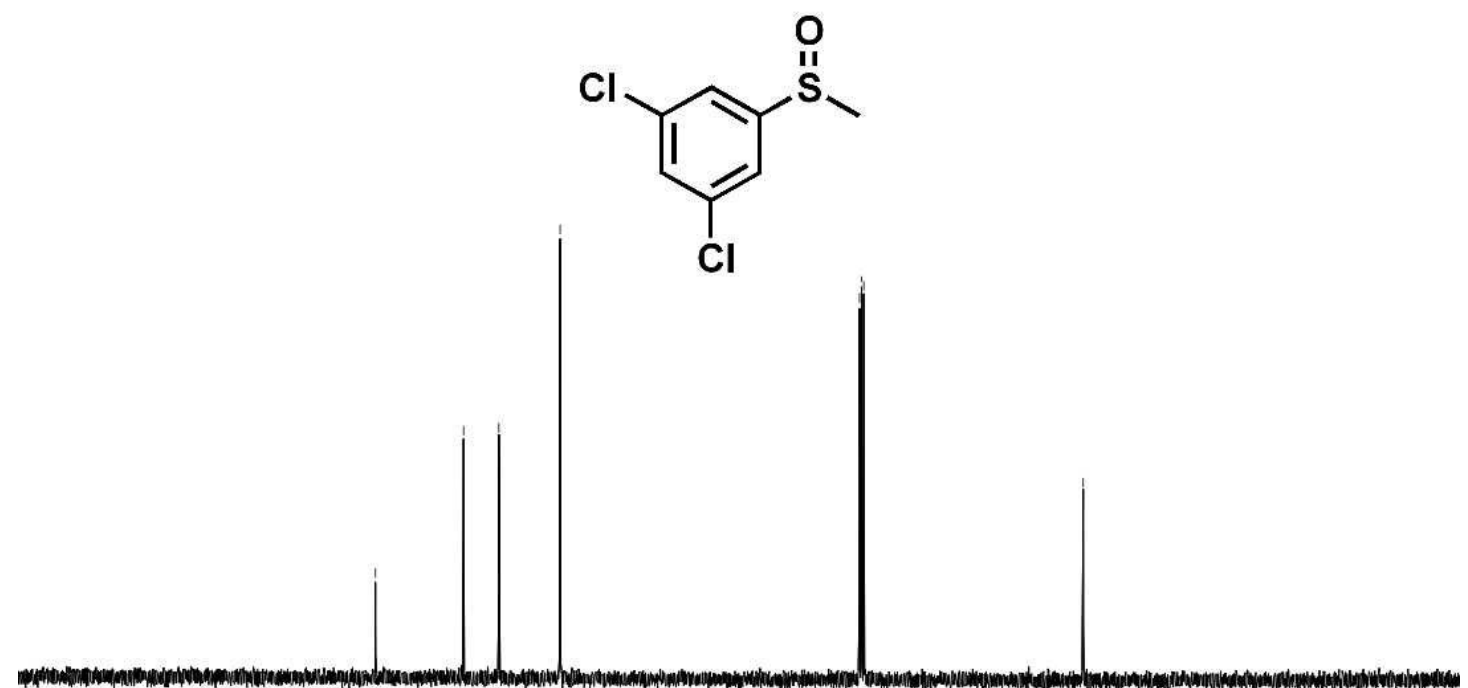

$\begin{array}{lllllllllllllllllllll}200 & 190 & 180 & 170 & 160 & 150 & 140 & 130 & 120 & 110 & \begin{array}{c}100 \\ \mathrm{f} 1(\mathrm{ppm})\end{array} & 80 & 70 & 60 & 50 & 40 & 30 & 20 & 10 & 0 & -10\end{array}$ 
${ }^{1} \mathrm{H}$ NMR of product 31 in $\mathrm{CDCl}_{3}(400 \mathrm{MHz})$

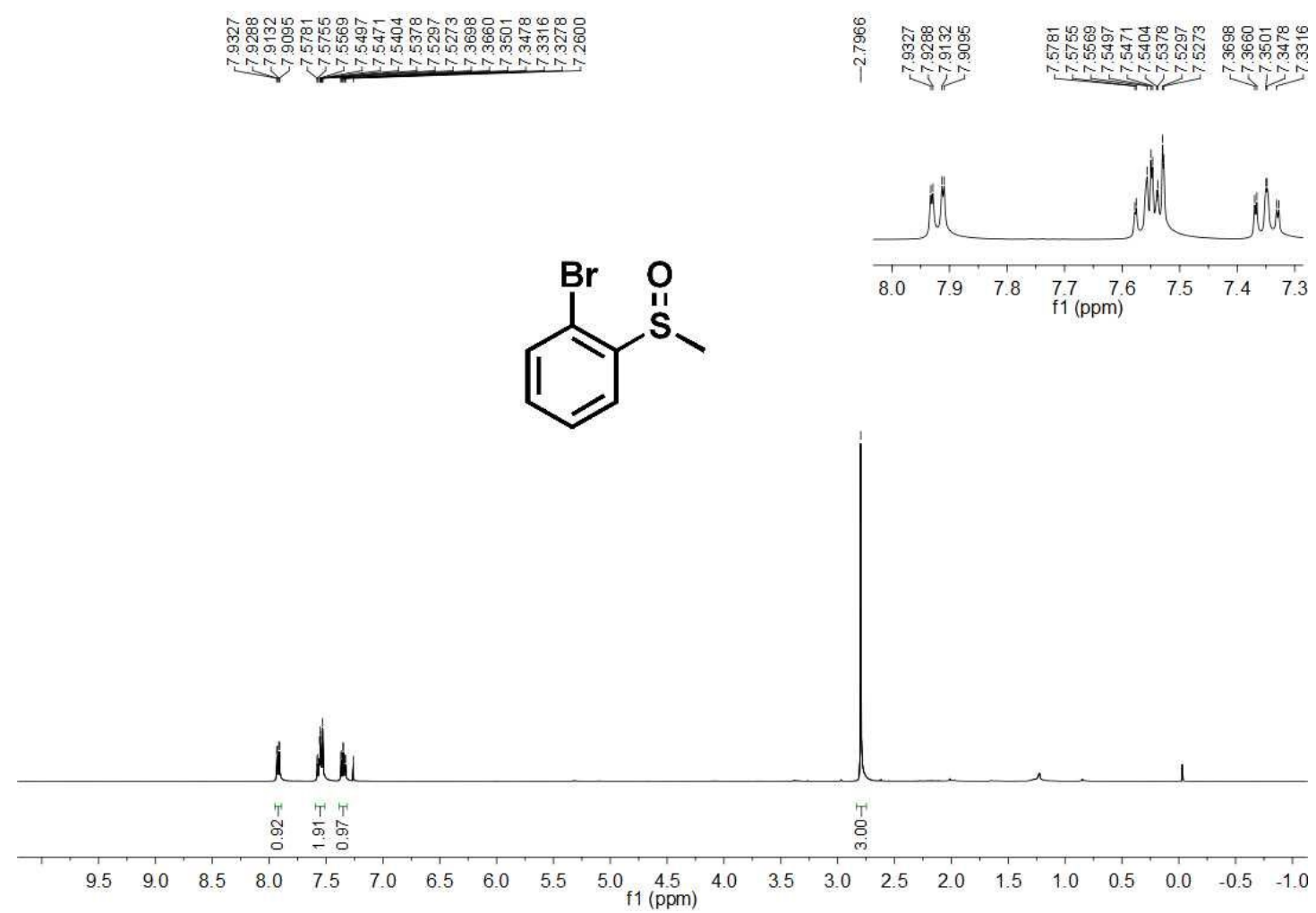

${ }^{13} \mathrm{C}$ NMR of product $3 \mathrm{I}$ in $\mathrm{CDCl}_{3}(100 \mathrm{MHz})$

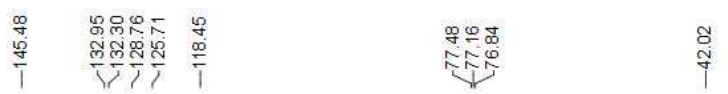<smiles>CS(=O)c1ccccc1Br</smiles>

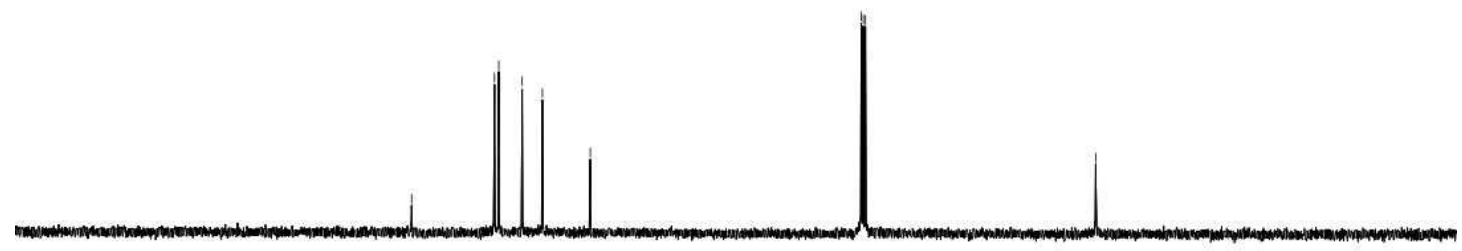

$\begin{array}{llllllllllllllllllllll}200 & 190 & 180 & 170 & 160 & 150 & 140 & 130 & 120 & 110 & \begin{array}{l}100 \\ \mathrm{f} 1(\mathrm{ppm})\end{array} & 80 & 70 & 60 & 50 & 40 & 30 & 20 & 10 & 0 & -10\end{array}$ 
${ }^{1} \mathrm{H}$ NMR of product $3 \mathrm{~m}$ in $\mathrm{CDCl}_{3}(400 \mathrm{MHz})$

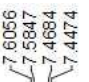

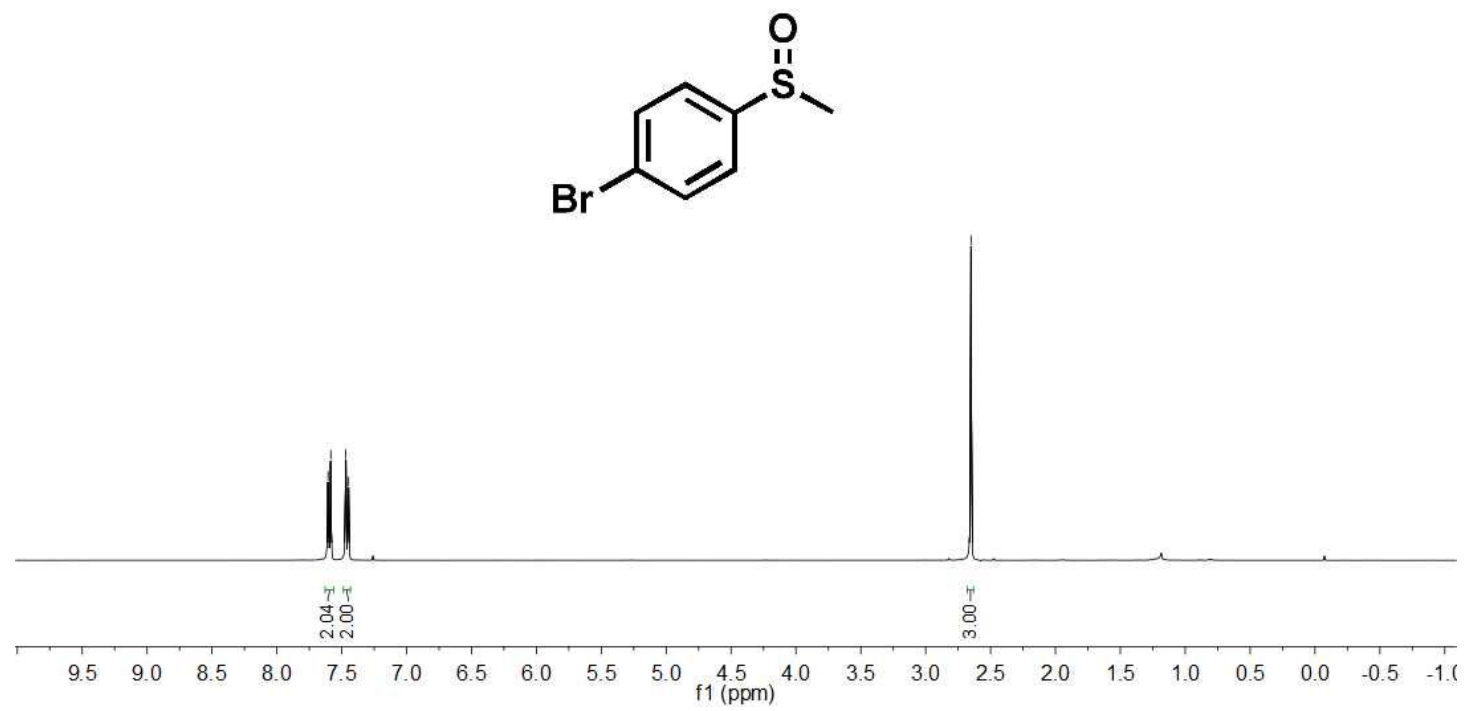

${ }^{13} \mathrm{C}$ NMR of product $3 \mathrm{~m}$ in $\mathrm{CDCl}_{3}(100 \mathrm{MHz})$

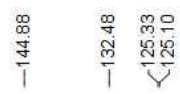

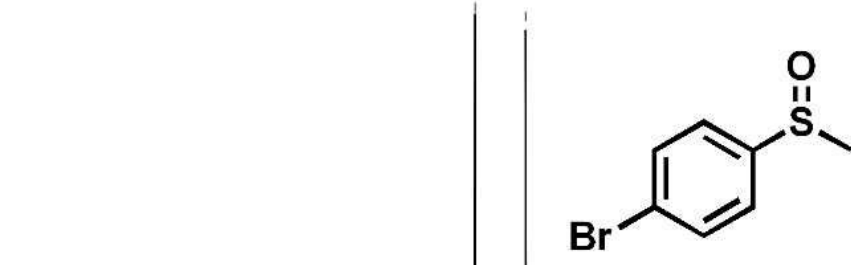

$\begin{array}{llllllllll}190 & 180 & 170 & 160 & 150 & 140 & 130 & 120 & 110 & \begin{array}{c}100 \\ \mathrm{f} 1(\mathrm{ppm})\end{array}\end{array}$

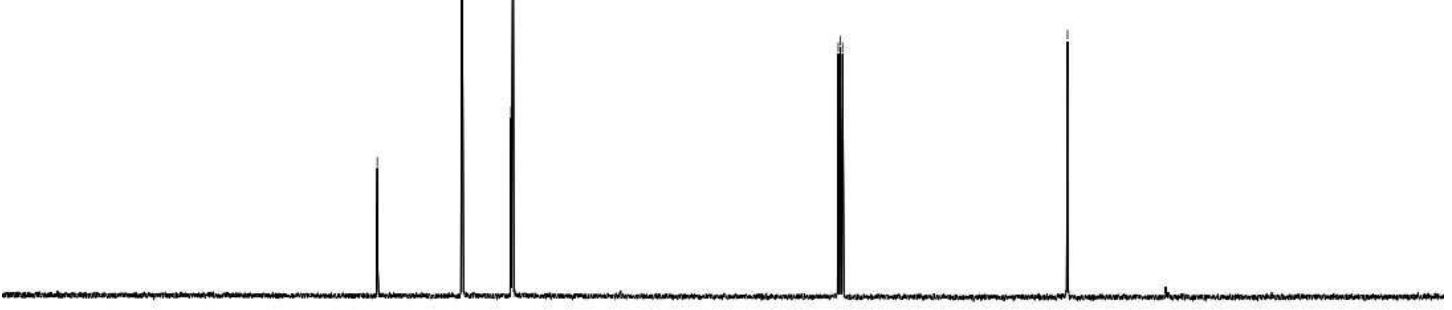

$\begin{array}{llllllllll}80 & 70 & 60 & 50 & 40 & 30 & 20 & 10 & 0 & -11\end{array}$ 
${ }^{1} \mathrm{H}$ NMR of product $3 \mathrm{n}$ in $\mathrm{CDCl}_{3}(400 \mathrm{MHz})$

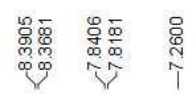<smiles>CS(=O)c1ccc([N+](=O)[O-])cc1</smiles>

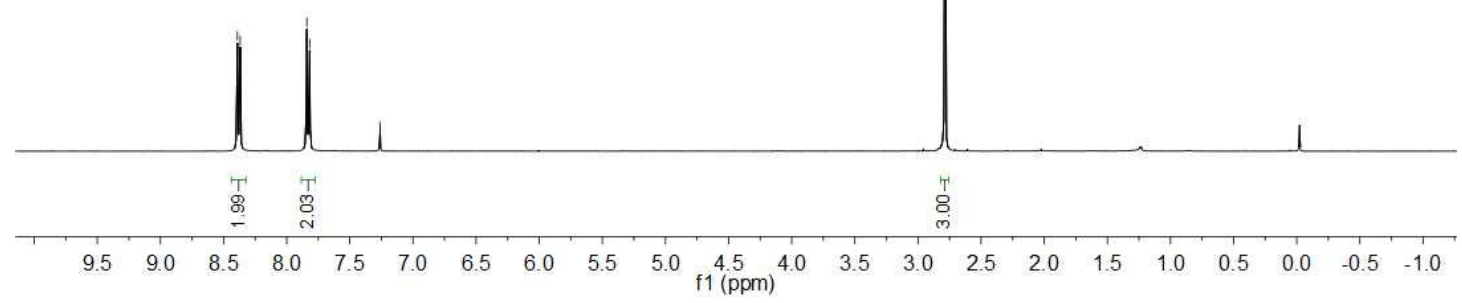

${ }^{13} \mathrm{C}$ NMR of product $3 \mathrm{n}$ in $\mathrm{CDCl}_{3}(100 \mathrm{MHz})$

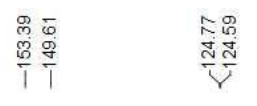

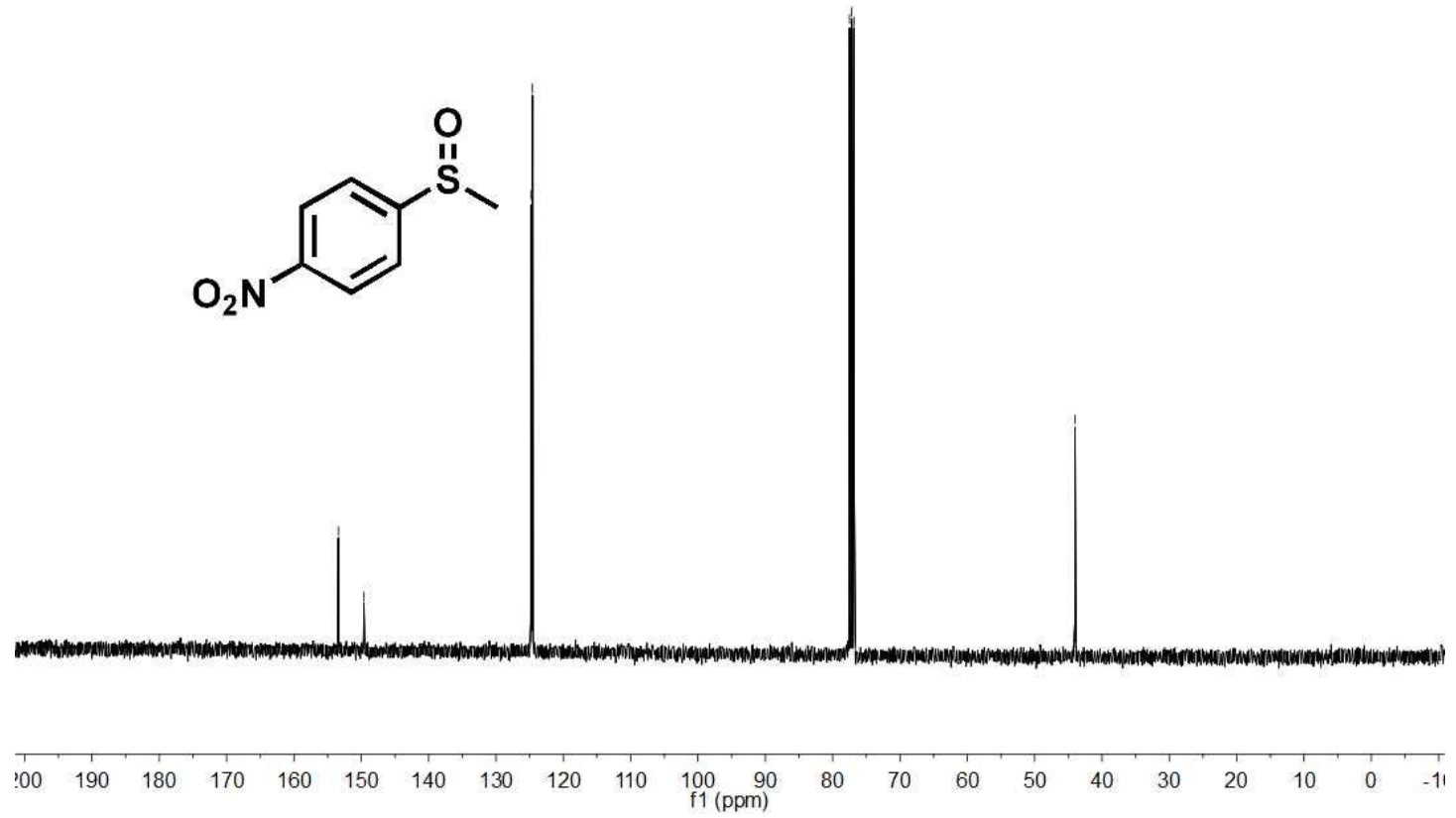


${ }^{1} \mathrm{H}$ NMR of product 30 in DMSO- $d_{6}(400 \mathrm{MHz})$

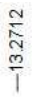

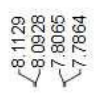
㠃怘

(c)

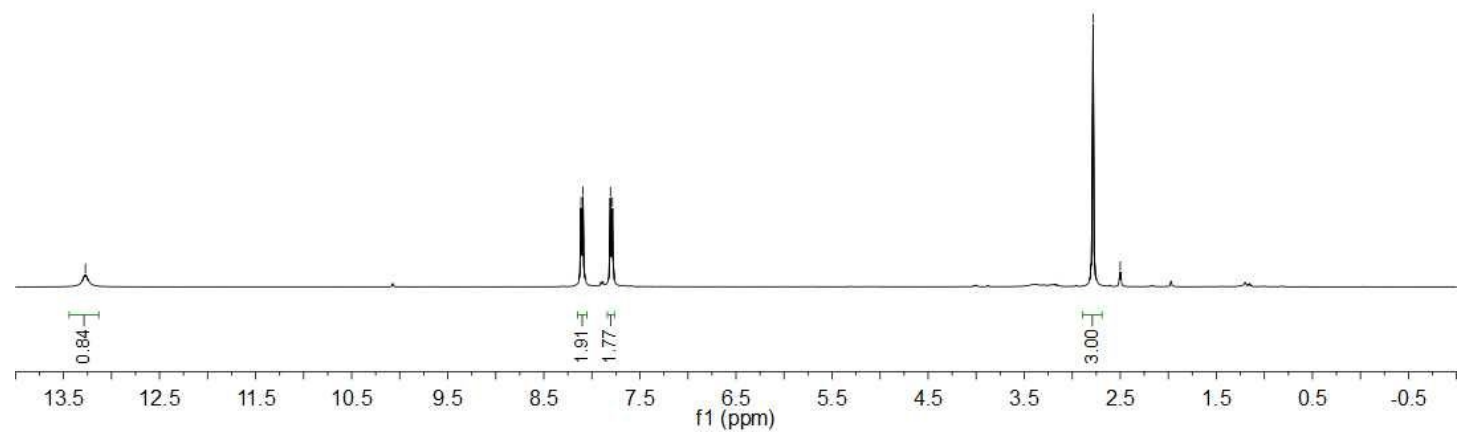

${ }^{13} \mathrm{C}$ NMR of product 30 in DMSO- $d_{6}(100 \mathrm{MHz})$<smiles>CS(=O)c1ccc(C(=O)O)cc1</smiles>

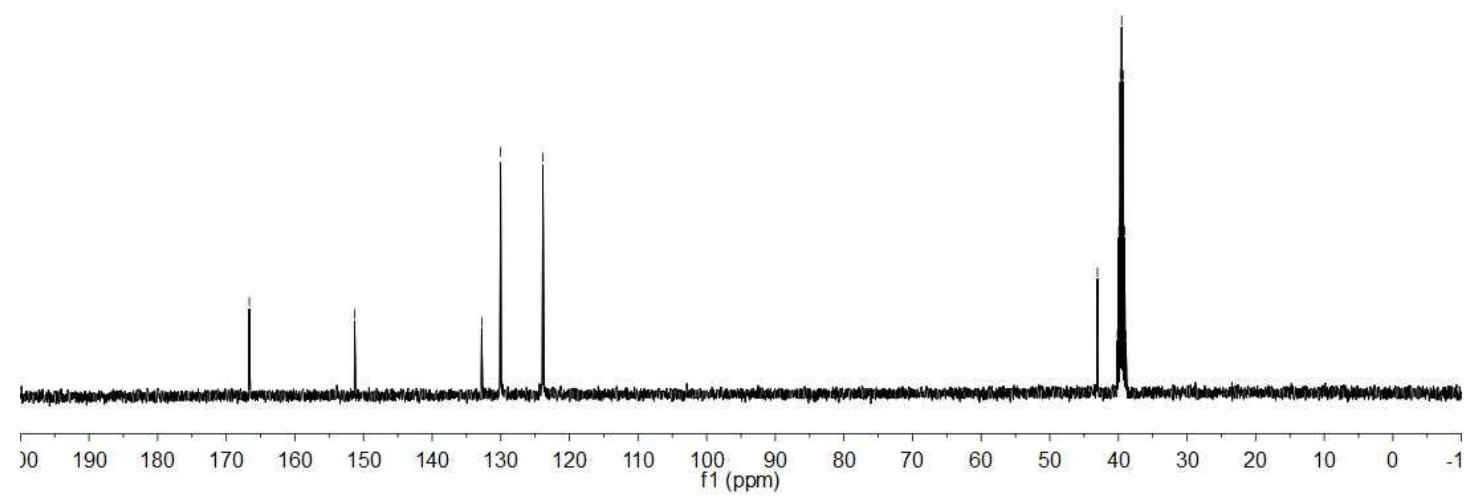


${ }^{1} \mathrm{H}$ NMR of product $3 p$ in $\mathrm{CDCl}_{3}(400 \mathrm{MHz})$
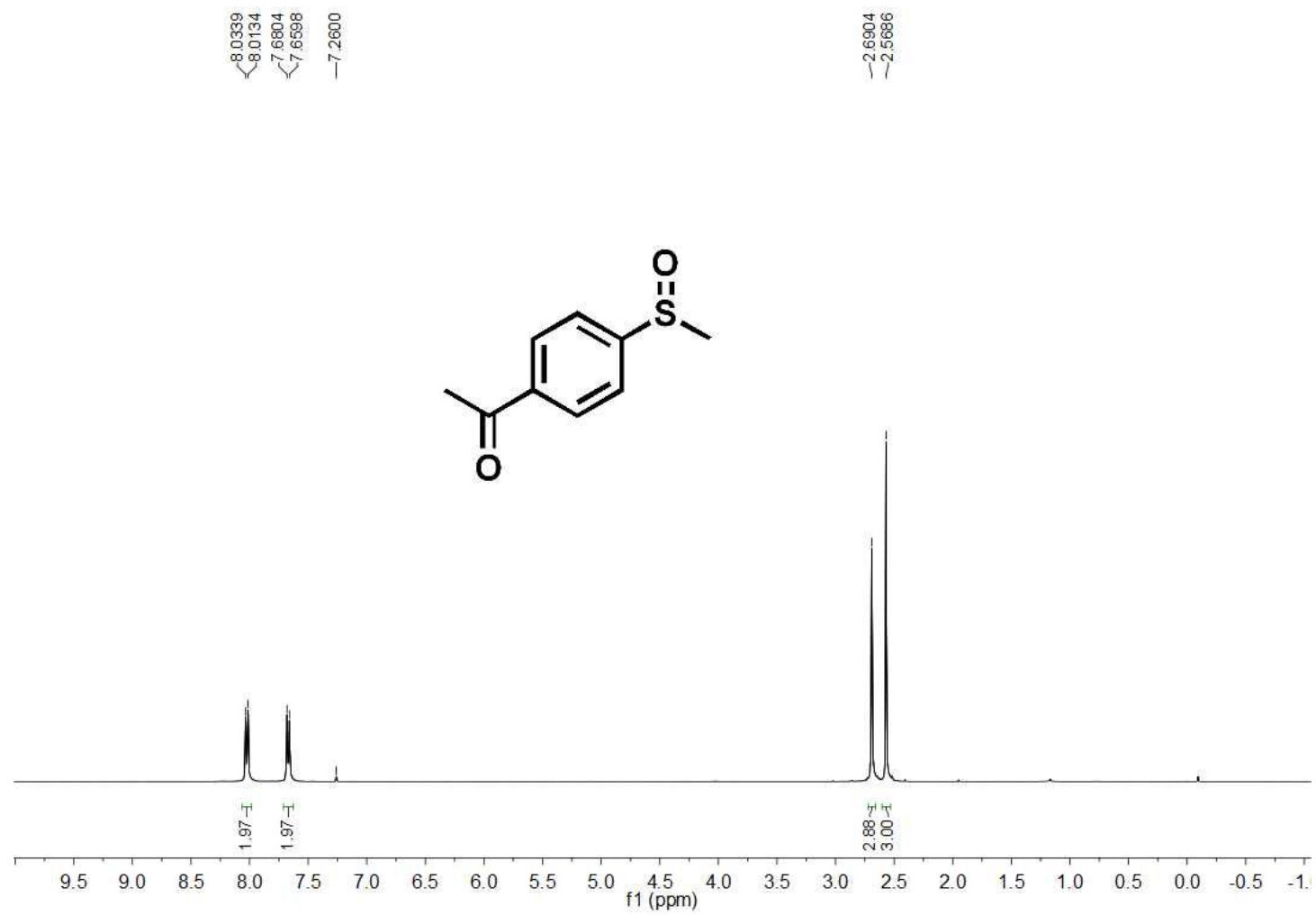

${ }^{13} \mathrm{C}$ NMR of product $3 p$ in $\mathrm{CDCl}_{3}(100 \mathrm{MHz})$
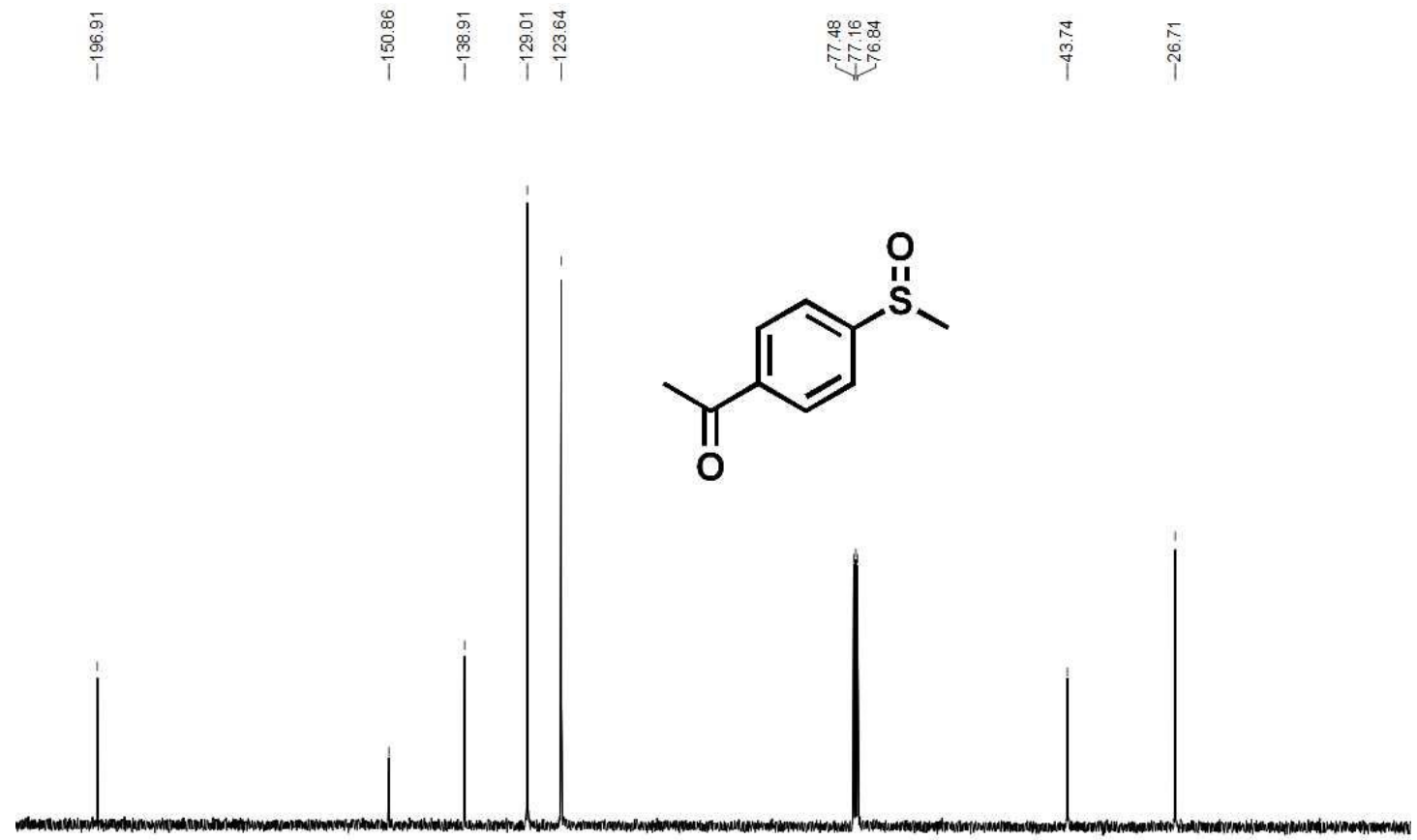

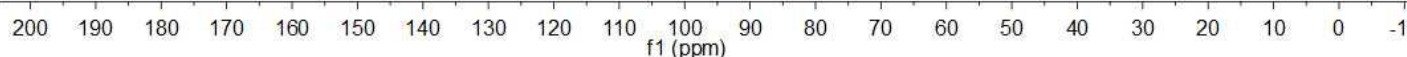


${ }^{1} \mathrm{H}$ NMR of product $3 q$ in $\mathrm{CDCl}_{3}(400 \mathrm{MHz})$
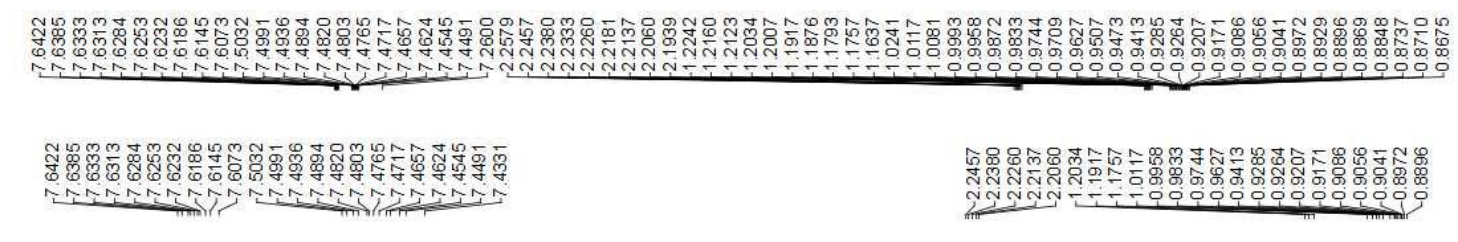

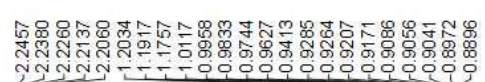

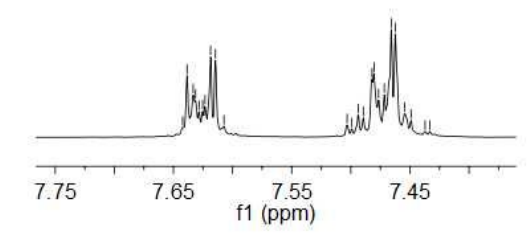<smiles>O=S(c1ccccc1)C1CC1</smiles>
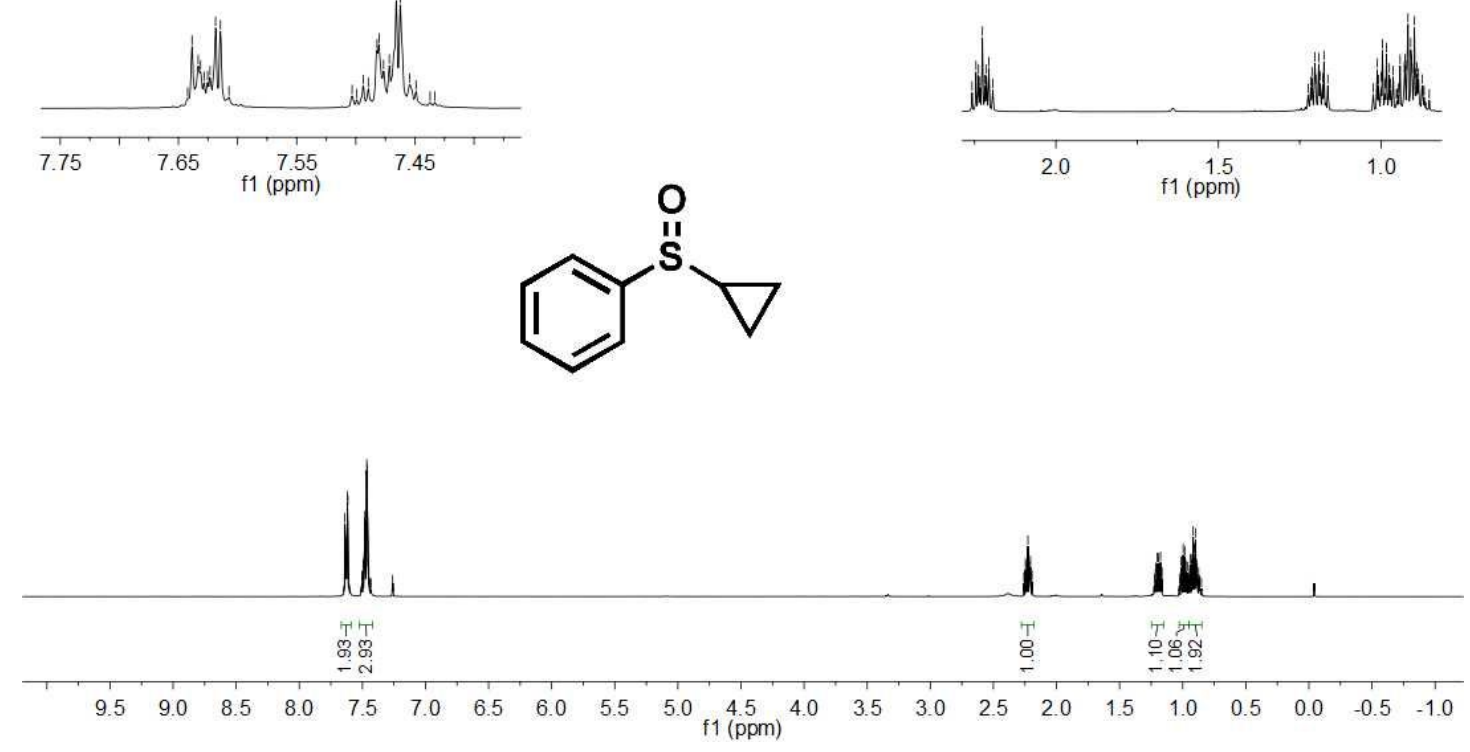

${ }^{13} \mathrm{C}$ NMR of product $3 q$ in $\mathrm{CDCl}_{3}(100 \mathrm{MHz})$

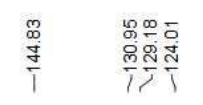

梁紫

$\stackrel{\bar{\infty}}{\prod_{p}^{p}}$

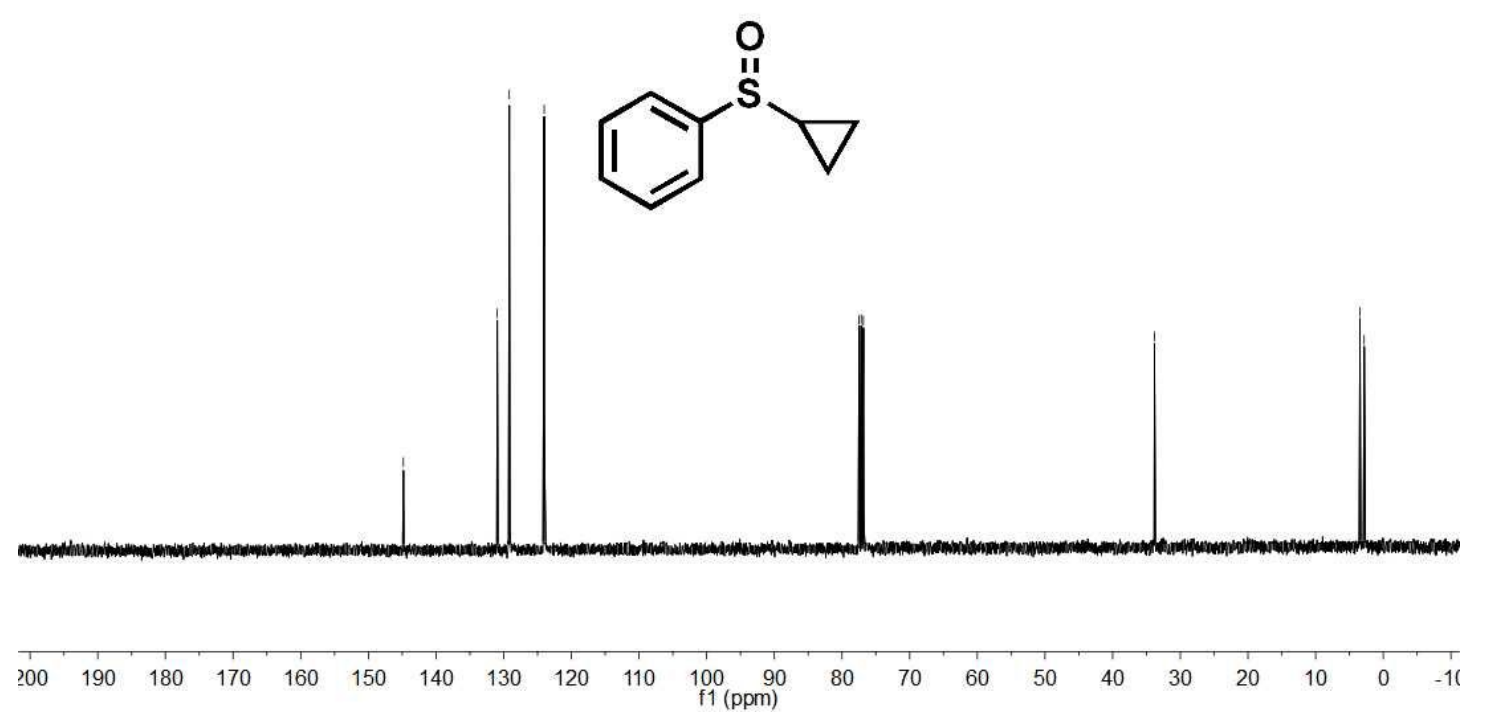


${ }^{1} \mathrm{H}$ NMR of product $3 \mathrm{r}$ in $\mathrm{CDCl}_{3}(400 \mathrm{MHz})$

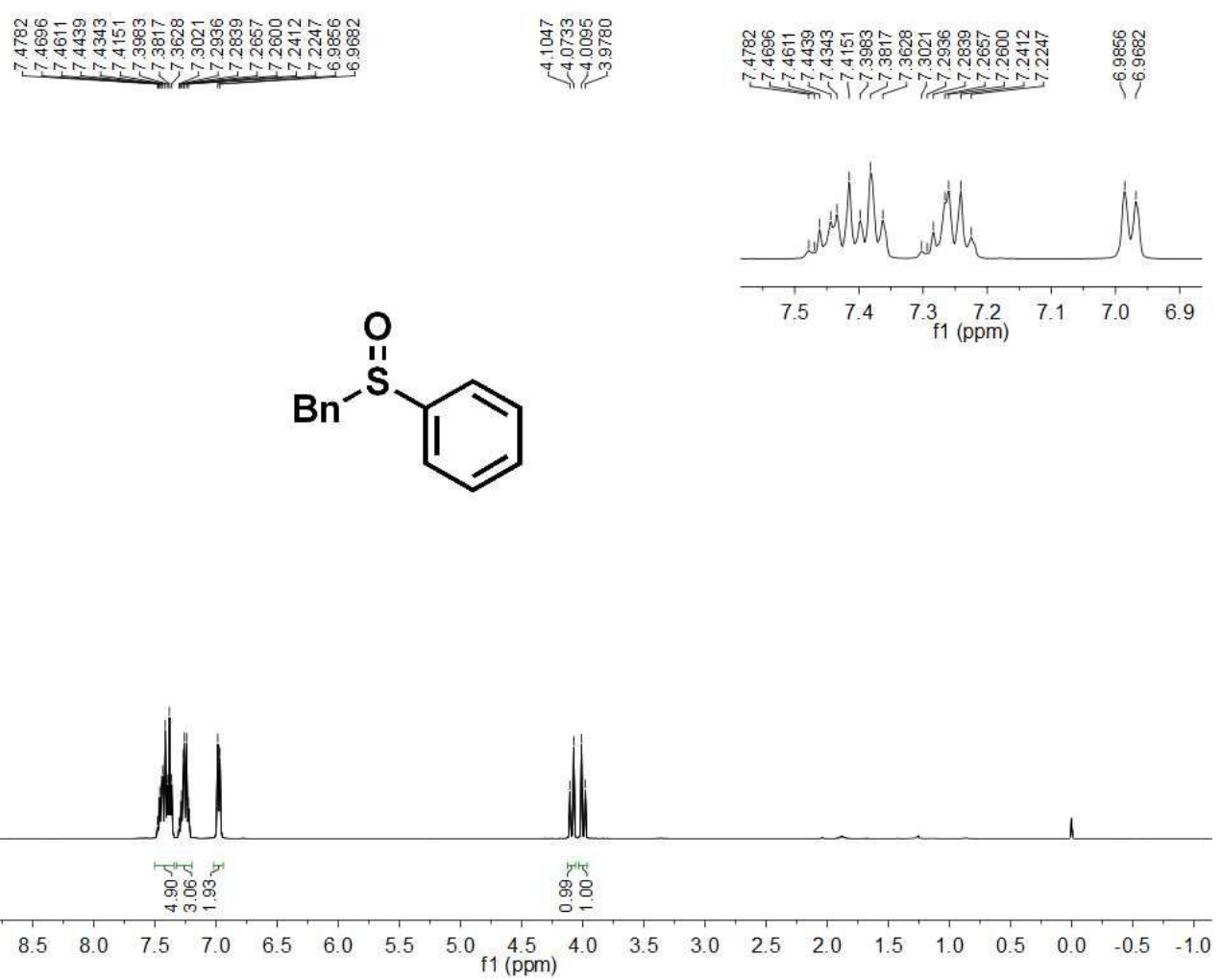

${ }^{13} \mathrm{C}$ NMR of product $3 \mathrm{r}$ in $\mathrm{CDCl}_{3}(100 \mathrm{MHz})$

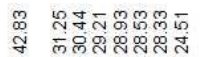

辛

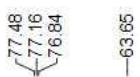

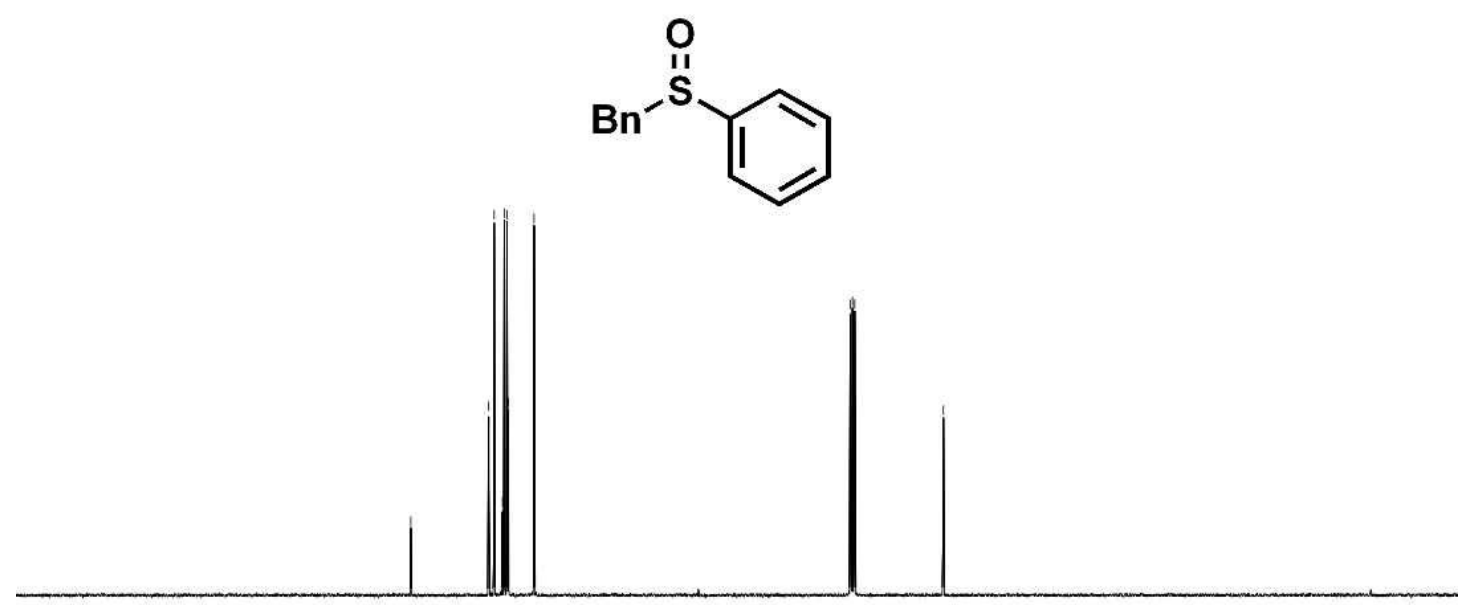

$\begin{array}{lllllllllllllllllllll}! 00 & 190 & 180 & 170 & 160 & 150 & 140 & 130 & 120 & 110 & \underset{f}{100} \mathbf{f}(\mathrm{ppm}) & 80 & 70 & 60 & 50 & 40 & 30 & 20 & 10 & 0 & -10\end{array}$ 
${ }^{1} \mathrm{H}$ NMR of product $3 \mathrm{~s}$ in $\mathrm{CDCl}_{3}(400 \mathrm{MHz})$

要

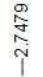

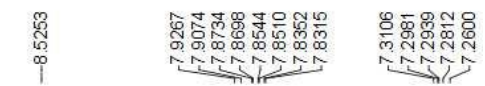<smiles>CS(=O)c1ccccn1</smiles>
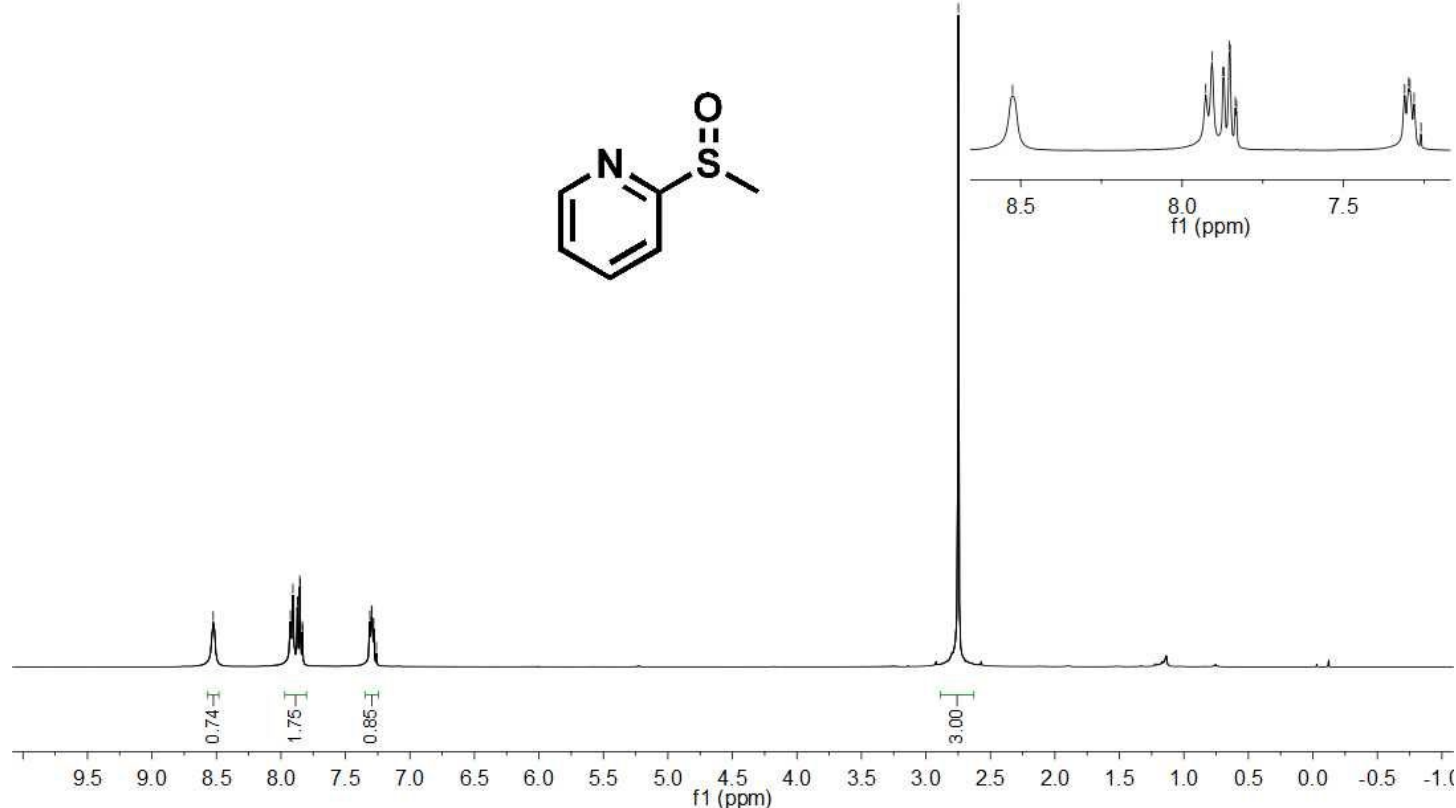

${ }^{13} \mathrm{C}$ NMR of product $3 \mathrm{~s}$ in $\mathrm{CDCl}_{3}(100 \mathrm{MHz})$

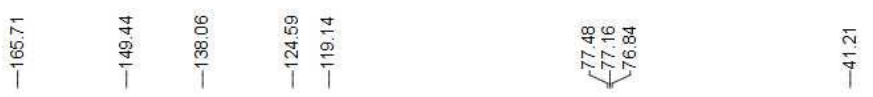<smiles>CS(=O)c1ccccn1</smiles>

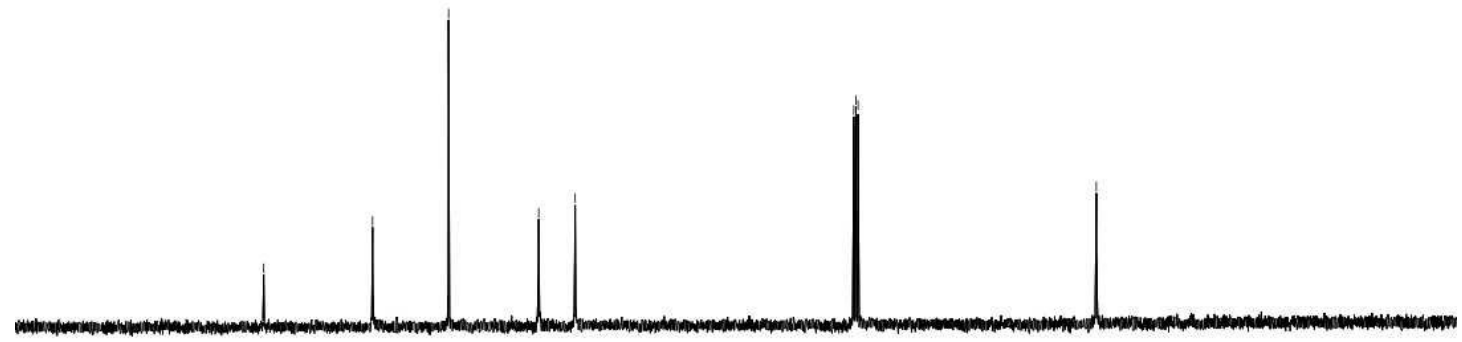

$\begin{array}{lllllllllllllllllllll}200 & 190 & 180 & 170 & 160 & 150 & 140 & 130 & 120 & 110 & \begin{array}{c}100 \\ \mathrm{f} 1(\mathrm{ppm})\end{array} & 80 & 70 & 60 & 50 & 40 & 30 & 20 & 10 & 0 & -10\end{array}$ 
${ }^{1} \mathrm{H}$ NMR of product $3 \mathrm{t}$ in $\mathrm{CDCl}_{3}(400 \mathrm{MHz})$

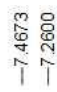<smiles>CS(=O)c1nc(Cl)cc(Cl)n1</smiles>

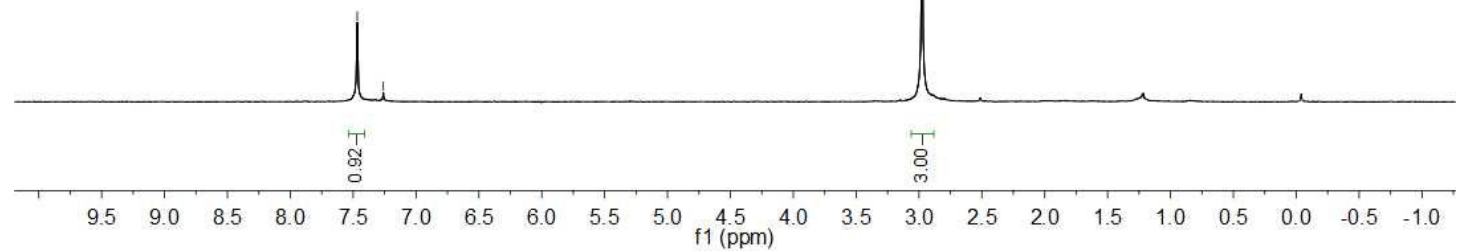

${ }^{13} \mathrm{C}$ NMR of product $3 \mathrm{t}$ in $\mathrm{CDCl}_{3}(100 \mathrm{MHz})$

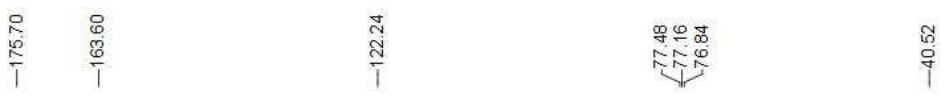

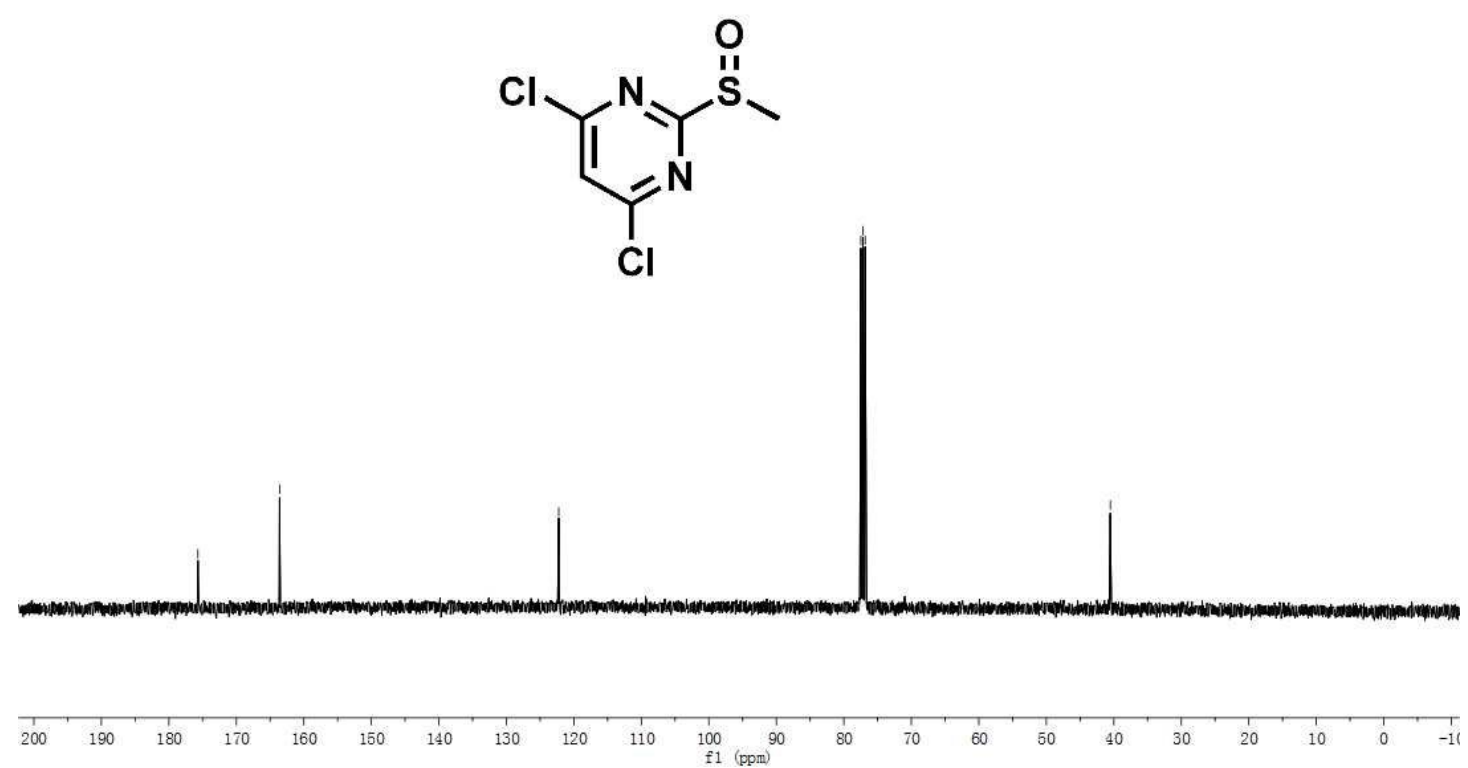


${ }^{1} \mathrm{H}$ NMR of product 4 in $\mathrm{CDCl}_{3}(400 \mathrm{MHz})$

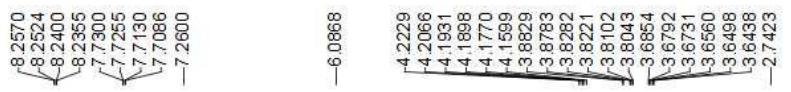<smiles>CS(=O)(=O)c1ccc(C(=O)OC2COCCO2)cc1</smiles>

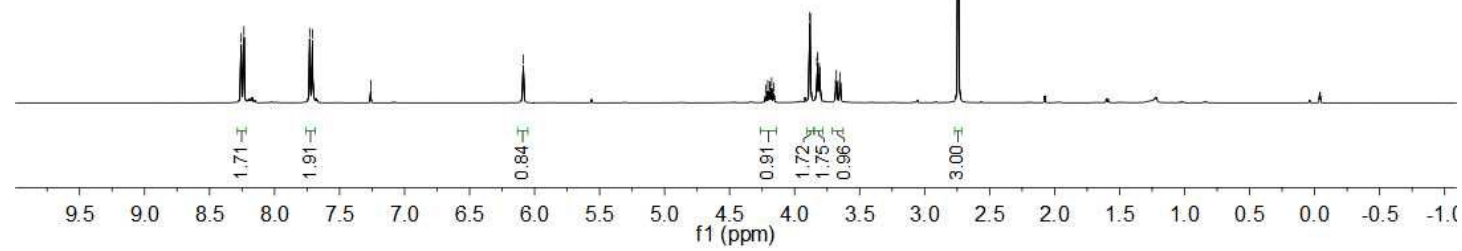

${ }^{1} \mathrm{H}$ NMR of product 5 in $\mathrm{CDCl}_{3}(400 \mathrm{MHz})$

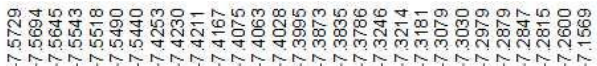

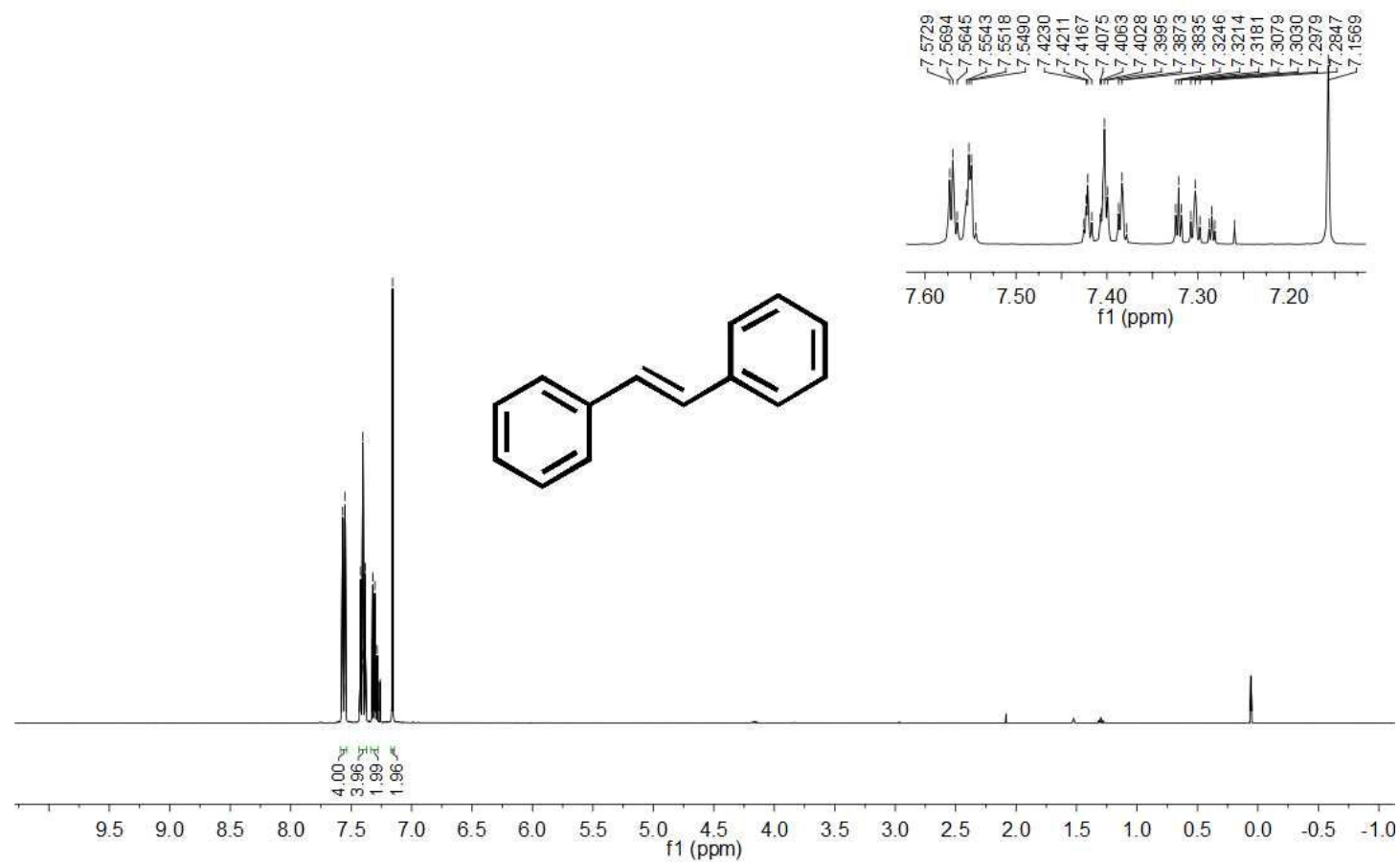


${ }^{1} \mathrm{H}$ NMR of product 6 in $\mathrm{CDCl}_{3}(400 \mathrm{MHz})$

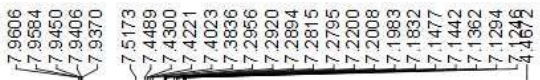

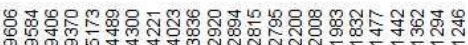

vis?<smiles>O=C(Cc1ccccc1Sc1ccccc1)c1ccccc1</smiles>
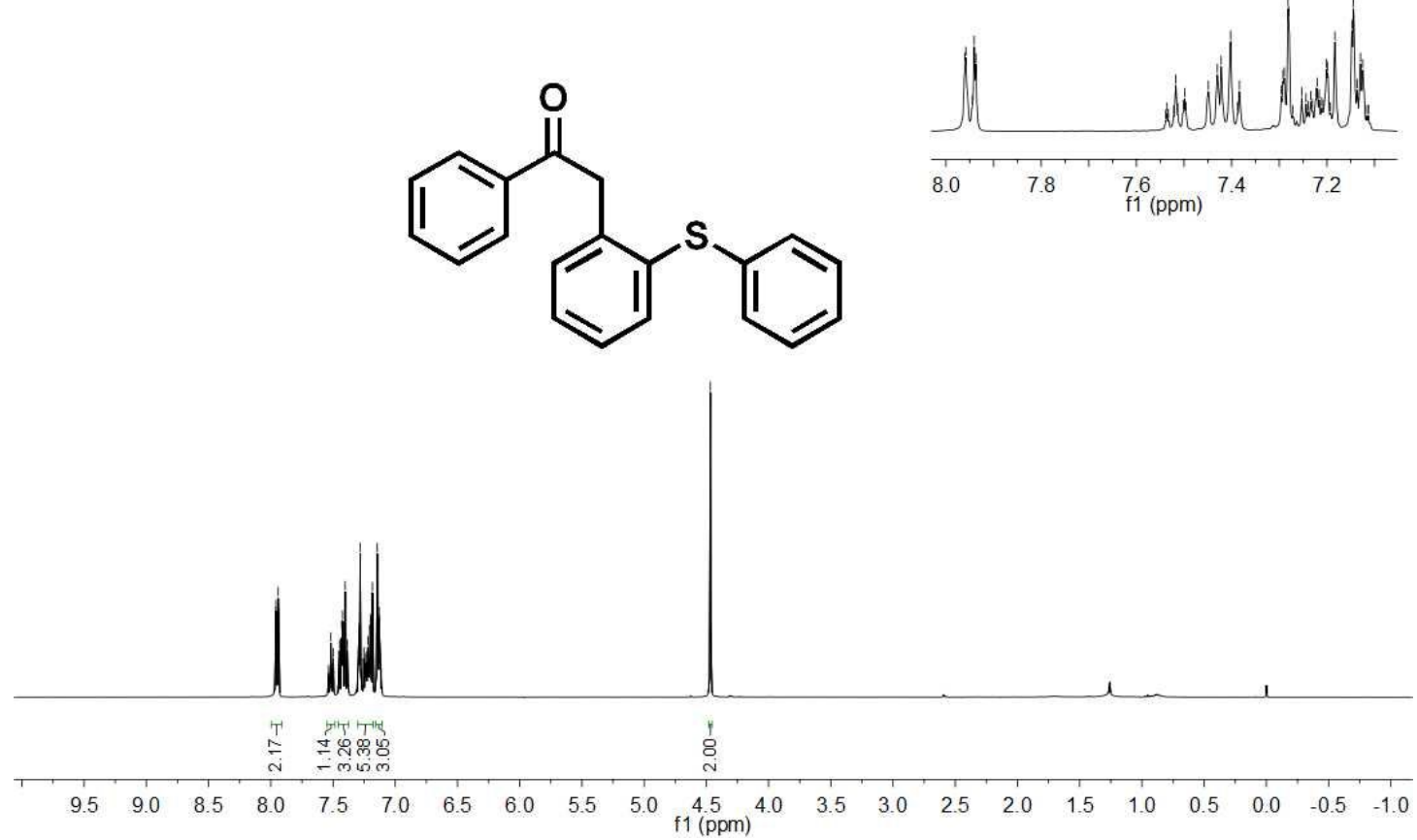

${ }^{1} \mathrm{H}$ NMR of product 7 in $\mathrm{CDCl}_{3}(400 \mathrm{MHz})$

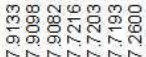

intan<smiles>O=C(c1ccccc1)[S+](c1ccccc1)c1ccccc1</smiles>

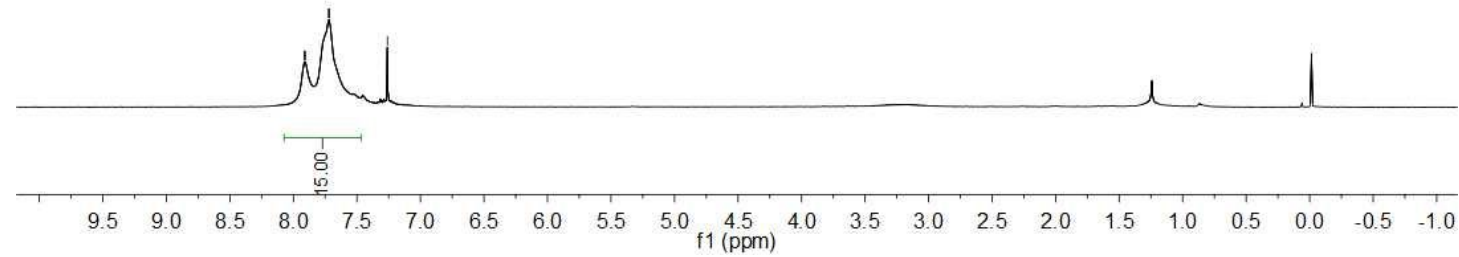

